\title{
ESTUDIO DEL POTENCIAL ÁTOMO-SUPERFICIE Y SU INFLUENCIA SOBRE LA ESTRUCTURA DE MONOCAPAS ADSORBIDAS
}

\author{
TESIS QUE PRESENTA \\ ALEXANDRE TKATCHENKO \\ PARA LA OBTENCIÓN DEL GRADO DE \\ DOCTOR EN CIENCIAS
}

28 DE MAYO DE 2007

UNIVERSIDAD AUTÓNOMA METROPOLITANA - IZTAPALAPA DIVISIÓN DE CIENCIAS BÁSICAS E INGENIERÍA

ASESOR:

DR. MARCELO E. GALVÁN ESPINOSA 


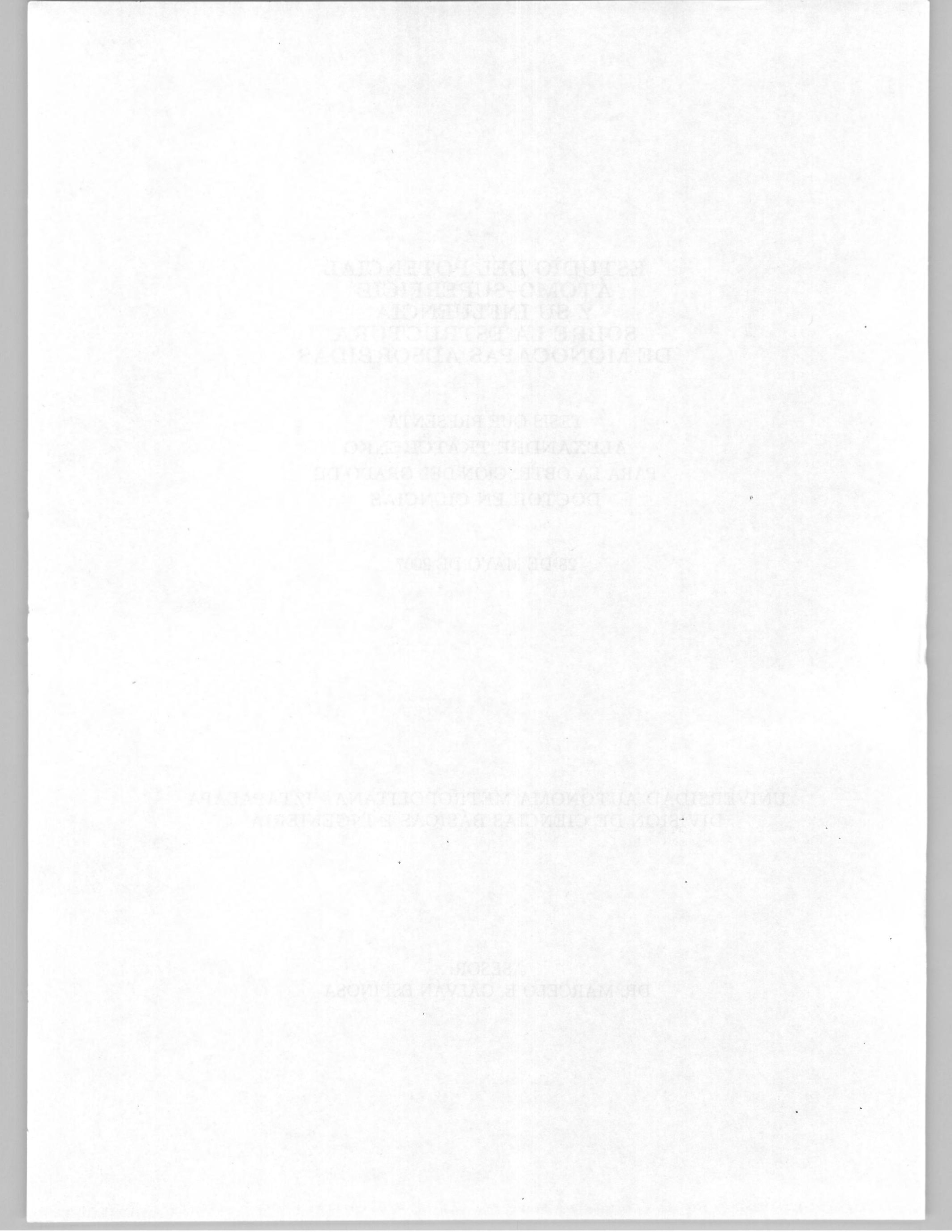


ESTA TESIS SE DESARROLLÓ EN EL ÁREA DE FISICOQUÍMICA TEÓRICA DEL DEPARTAMENTO DE QUÍMICA

BAJO LA DIRECCIÓN DEL DR. MARCELO E. GALVÁN ESPINOSA

EL EXAMEN DE GRADO SE LLEVÓ A CABO EL DIA 28 DE MAYO DE 2007 A LAS 11:30 HORAS EN LA UNIVERSIDAD AUTÓNOMA METROPOLITANA IZTAPALAPA

EL JURADO ESTUVO FORMADO POR LOS SIGUIENTES SINODALES:

DR. SALVADOR A. CRUZ JIMÉNEZ - PRESIDENTE

DR. JOSE ANDRÉS CEDILLO ORTIZ - SECRETARIO

DR. RODOLFO ESQUIVEL OLEA - VOCAL DR. WOLF LUIS MOCHÁN BACKAL - VOCAL DRA. MARCELA BELTRÁN SÁNCHEZ - VOCAL 


\section{Agradecimientos}

Mucha gente ha contribuido, directamente o indirectamente, al desarrollo de esta tesis. En primer lugar, quisiera agradecer a mi familia por el apoyo incondicional y por impulsarme a ser mejor en todos los aspectos personales y profesionales.

Sin duda, la investigación realizada a través de los últimos cuatro años no hubiera sido posible sin la dirección y el apoyo de mi asesor, el Dr. Marcelo Galván. Gracias, Marcelo, por darle una ruta a mi investigación y, a la vez, dejarme ser libre para seguir mi propio camino. Asímismo, no se puede olvidar que el "culpable" de la temática original de esta tesis es el Dr. Nikola Batina, quien ha sido un excelente amigo, maestro y colaborador a lo largo de muchos años. Gracias, Nikola, por tus consejos y enseñarme que puedo lograr cualquier cosa que me proponga. Quisiera agradecer a mi colega, el Dr. Anatole von Lilienfeld, por horas de interesantes y productivas discusiones y por enseñarme a usar $\mathrm{INT}_{\mathrm{E}} \mathrm{X}$ el cual fue usado para escribir esta tesis. Otra gran amiga mía es María de la Luz Benitez, quien me enseño el "mundo real" y me permitió ser su colaborador a lo largo de los últimos seis años. Gracias, Lucy, por darme otra visión en mi carrera profesional. Por otro lado, agradezco al Dr. Alberto Vela Amieva por darme la oportunidad de desarrollarme en el campo de clusters Linux y al Dr. Carlos Bunge Molina por introducirme al uso eficiente de la Computación en la Física y por su genuina preocupación en cuanto al futuro de mi carrera científica. Asímismo, agradezco a los profesores (Rubi, Jorge, Andrés y Jose Luis) y los alumnos y exalumnos (Felipe, Myrna, Edmundo, Nelly, Alvaro, Zeferino, Juan y Alejandra entre otros) 
del área de Fisicoquímica Teórica del Departmento de Química de la Universidad Autónoma Metropolitana Unidad Iztapalapa por su apoyo, así como al Ing. Juan Carlos Rosas Cabrera por su amistad y colaboración en cuestiones de cómputo de alto rendimiento.

Quisiera expresar mi gratitud al Dr. Andrés Cedillo Ortiz, al Dr. Rodolfo Esquivel Olea, al Dr. Salvador Cruz Jiménez, al Dr. Luis Mochan Backal y a la Dra. Marcela Beltrán Sánchez por la revisión de esta tesis y sus valiosos comentarios.

Por últímo, agradezco a la UAM-Iztapalapa por sus instalaciones del más alto nivel en cómputo (Laboratorio de Supercómputo y Visualización en Paralelo) y el apoyo económico por parte del CONACyT para realizar los estudios de doctorado. 
A mi familia (I. T., M. T., A. T., F. R. P. y L. T. R.) 



\section{Resumen}

El propósito de esta tesis es la investigación teórica de estructuras ordenadas de monocapas hexagonales adsorbidas en superficies con simetría fcc(111) y, en particular, la aplicación de estos resultados al estudio de monocapas ordenadas de yodo sobre superficies metálicas. El ingrediente más importante para este estudio es un análisis detallado del potencial de interacción átomo-superficie. Se hace uso de distintos métodos para alcanzar los objetivos planteados en esta tesis; desde la descripción geométrica de superficies con modelos matemáticos, teorías analíticas de monocapas infinitas, hasta cálculos basados en la Teoría de Funcionales de la Densidad (TFD) para analizar la energética fina de la adsorción de yodo. Uno de los objetivos más importantes es lograr una retroalimentación entre los métodos sencillos de descripción de adsorción en superficies y las simulaciones de primeros principios. En todos los casos analizados, los resultados de este estudio se comparan con los datos experimentales constatando que se obtiene un buen acuerdo entre la teoría y el experimento. 



\section{Índice general}

Índice general

1. Antecedentes $\quad 5$

1.1. Superficies: simetría y sitios $\ldots \ldots \ldots \ldots$

1.2. Notación de Wood para estructuras en superficies . . . . . . . . . 8

1.3. Potenciales de interacción ..................... 8

1.4. Modelos para comportamiento epitaxial . . . . . . . . . . . . . 12

2. Monocapa rígida hexagonal 17

2.1. Monocapas hexagonales: consideraciones de simetría . . . . . . . . . . . 17

2.2. Aproximación de monocapa rígida . . . . . . . . . . . . . 21

2.3. Teoría analítica de monocapa rígida hexagonal infinita . . . . . . . . 23

2.4. Relación de la expansión de Fourier con los "números mágicos" . . . . . . . 36

3. Simulaciones de monocapas de halógenos con TFD 41

3.1. Metodología TFD ......................... 41

3.2. Un átomo en las celdas $(2 \times 2)$ y $(3 \times 3) \ldots \ldots \ldots \ldots$

3.2.1. Partición de Hirshfeld $[84] \ldots \ldots \ldots$. . . . . . . . . 46

3.2.2. Modelo de superficie ................... 47

3.2.3. Resultados .......................... 48 
3.2.4. Discusión ............................ 53

3.3. Estructuras ordenadas de I-Pt(111) . . . . . . . . . . . 56

3.3.1. Metodología ..................... 57

3.3.2. Resultados ...................... 59

3.3.3. Discusión ......................... 63

4. Análisis combinado del sistema (3×3)-I-Pt(111) 65

4.1. Planteamiento del problema .................... 65

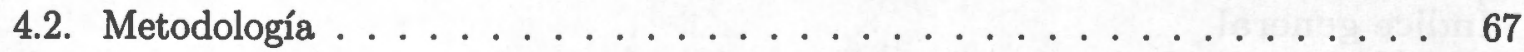

4.2.1. Cálculos TFD . . . . . . . . . . . . . . . 67

4.2.2. Simulaciones Monte Carlo (MC) . . . . . . . . . . . 68

4.3. Resultados y Discusión . . . . . . . . . . . . . . . . 69

$\begin{array}{lr}\text { A. Publicaciones del autor } & \mathbf{8 1}\end{array}$

$\begin{array}{lr}\text { Bibliografía } & \mathbf{8 3}\end{array}$ 


\section{Introducción}

Everything you touch is a surface.

- Anónimo

Como lo dice el epígrafe, todo lo que se toca es una superficie. Por esta razón, el estudio de fenómenos en superficies se puede remontar a los años 1700 , desde la investigación del esparcimiento de aceite sobre agua por Benjamin Franklin [1] y los comienzos de la catálisis en el siglo XVIII [1,2]. Sin embargo, el gran empuje en la ciencia de superficies como tal se dió a partir de la decada de 1950 a 1960 con la invención de técnicas especializadas para distinguir los arreglos atómicos a nivel microscópico. Muchas de las técnicas desarrolladas originalmente para estudios de fenómenos en superficies, en la actualidad también se utilizan para estudiar materiales en bulto.

Hoy en día, la contínua miniaturización de componentes electrónicos y el afán de manipular objetos ordenados a un nivel cada vez más cercano al régimen atómico le da una particular importancia al estudio de arreglos formados por átomos y moléculas en superficies con un orden traslacional definido. Sobre todo, la gran cantidad de datos experimentales disponibles para monocapas sobre superficies metálicas permite y demanda efectuar estudios teóricos para explicar y racionalizar distintos comportamientos e identificar tendencias. Como un ejemplo, se puede mencionar la gran variedad de comportamientos epitaxiales de monocapas sobre superficies, para muchos de los cuales no se ha encontrado una explicación teórica. En gran parte esto se debe a que los potenciales de interacción para sistemas 
monocapa-superficie no se conocen a detalle. De hecho, las expresiones analíticas para estos potenciales se conocen solamente en casos aislados, por ejemplo para algunos gases nobles en superficies metálicas. En un gran número de casos, ni siquiera se conoce el orígen de las interacciones.

Al contrario, en el caso de sistemas como cúmulos atómicos, moléculas biológicas o vidrios, los potenciales de interacción se conocen con cierta precisión. Esto ha hecho posible un estudio exhaustivo de la superficie de energía potencial (SEP) y ha permitido conocer a fondo las estructuras de mínima energía y la dinámica de estos sistemas [3]. Por lo anterior creemos que el estudio de potenciales de interacción en sistemas monocapa-superficie permitirá hacer algo similar y avanzar el estado del arte en la investigación de los arreglos atómicos o moleculares en superficies ordenadas.

Recientemente se ha vuelto rutinaria la investigación de fenómenos de adsorción por medio de cálculos basados en la Teoría de Funcionales de la Densidad (TFD) y han ido disminuyendo paulatinamente los estudios sobre sistemas monocapa-superficie con modelos analíticos. Los cálculos de TFD permiten, en principio, obtener geometrías y energías confiables para una gran variedad de sistemas, por lo que se pueden hacer comparaciones directas con los datos experimentales. Sin embargo, los resultados obtenidos para un sistema específico no se pueden extrapolar a ningún otro sistema. Aunque los parámetros de reactividad dentro de la TFD permiten hacer algunas extrapolaciones y proponer tendencias, su aplicación a sistemas de estado sólido ha sido limitada. Por otro lado, el costo computacional de los cálculos de TFD limita los estudios detallados de la superficie de energía potencial de un sistema dado. Es por eso que en el presente trabajo queremos enfatizar que los cálculos de primeros principios (TFD) y modelos analíticos son complementarios y la conjunción de estos dos enfoques puede llevar a hallazgos importantes.

Dentro de la ciencia básica de superficies, muchos sistemas han servido como modelos. Tal es el caso de los gases nobles ( $\mathrm{Ar}, \mathrm{Kr}$ y $\mathrm{Xe}$ ) en superficies metálicas [4] como ejemplo 
de fisisorción y metales alcalinos para quimisorción [5]. Otro tipo de sistemas que ha sido estudiado extensivamente por distintos métodos experimentales es la adsorción de halógenos ( $\mathrm{I}, \mathrm{Br}$ y Cl) en superficies metálicas de platino, oro y plata entre otros. Para este último caso existe un número reducido de estudios teóricos confiables (v.g., de primeros principios).

Gran parte de esta tesis versará sobre las monocapas de yodo adsorbido en la superficie Pt(111). Esta elección se debe a que existe un número grande de estudios experimentales con diferentes técnicas de caracterización sobre este sistema, sin embargo los estudios teóricos aún son escasos. El estudio de este sistema en particular permitió darnos cuenta de la importancia del análisis detallado del potencial átomo-superficie y cómo este afecta la estabilidad de estructuras monocapa-superficie.

En el primer capítulo se revisan los conceptos básicos de la estructura de sistemas monocapa-superficie y las interacciones presentes en estos sistemas.

En el segundo capítulo, se propone un esquema sencillo de clasificación de las estructuras de monocapas hexagonales en superficies con simetría (111). Se demuestra que la estructura algebráica relacionada con esta clasificación tiene una clara asociación con los términos de Fourier del poteńcial átomo-superficie, lo que permite predecir estructuras de monocapas adsorbidas estables con el sólo conocimiento del potencial de interacción átomo-superficie. Así mismo, se presentan algoritmos que permiten construir un sistema monocapa-superficie conmensurable con un número de partículas dado, lo que resulta ser de gran ayuda para la construcción de modelos geométricos para cálculos de estructura electrónica.

En el tercer capítulo se presentan los resultados de cálculos basados en la TFD, que permiten estudiar la estructura electrónica de sistemas sustrato-adsorbato de un tamaño (número de átomos) restringido. Esto nos permite conocer a detalle el potencial de interacción átomo-superficie para sistemas de nuestro interés y hacer algunas especulaciones sobre la naturaleza de las interacciones átomo-átomo en la monocapa adsorbida. 
En el cuarto y último capítulo se plantea el siguiente cuestionamiento: $i$ Por que las estructuras que se observan experimentalmente en distintos ambientes (vacio, aire y solución) son diferentes para el sistema (3×3)-I-Pt(111) ? Utilizamos una metodología combinada de cálculos de TFD y Monte Carlo para analizar en detalle la superficie de energía potencial del sistema (3×3)-I-Pt(111) con un modelo sencillo de interacciones. Como se verá, esto nos permite encontrar una posible explicación para la diferencia en estructuras observadas como función de un solo parámetro que controla la repulsión entre los átomos de la monocapa adsorbida.

Finalmente, presentamos las conclusiones de esta tesis y las perspectivas de trabajo a futuro en las dos últimas secciones. 


\section{Capítulo 1}

\section{Antecedentes}

En este capítulo se presentan los conceptos y la terminología básica utilizada en el estudio de monocapas adsorbidas en superficies ordenadas. Empezamos por describir los distintos tipos de superficies del cristal cúbico centrado en las caras (f.c.c. por sus siglas en inglés). Continuamos con la presentación de la nomenclatura de distintos sitios de adsorción en una superficie (111), la cual se va a estudiar en detalle a través de esta tesis junto con la notación de Wood utilizada para describir los arreglos de monocapas en superficies ordenadas. Después se presentan los distintos tipos de modelos utilizados anteriormente para describir las estructuras de una monocapa sobre una superficie ordenada y para la descripción de interacciones en estos sistemas. Al final, se trata de proporcionar un panorama breve del estado del arte en la investigación del comportamiento epitaxial de sistemas monocapasuperficie y explicar a grandes rasgos cual es la contribución que realiza el presente trabajo al mismo. 


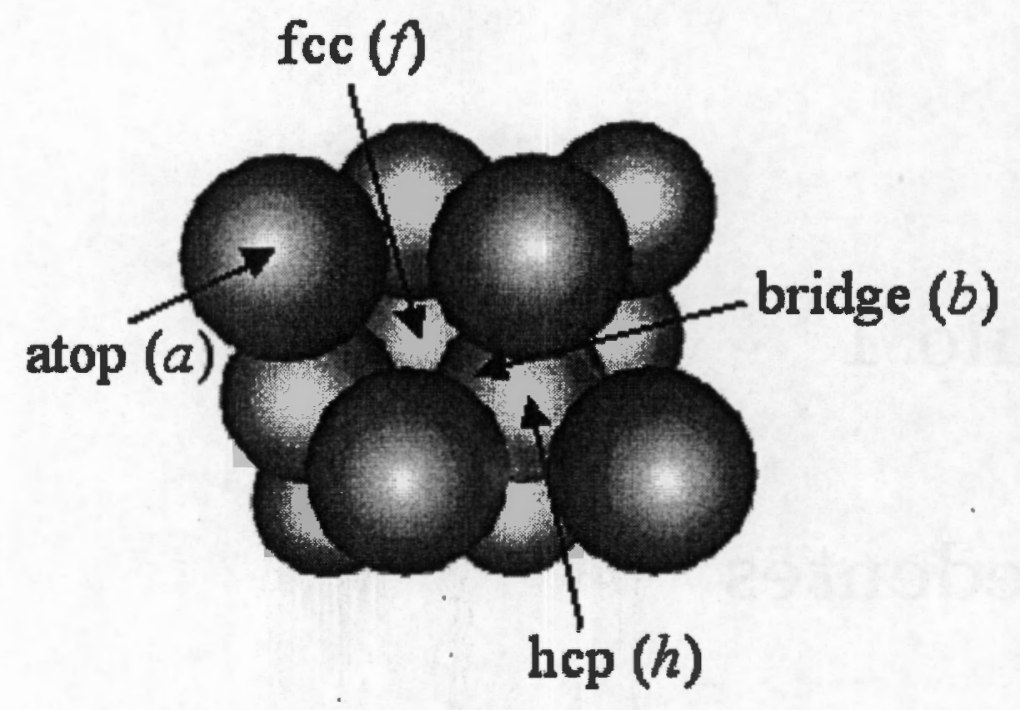

Figura 1.1: Modelo finito de la superficie (111) (empaquetamiento $A B C$ ), mostrando tres capas hacia abajo. Los sitios más simétricos se indican con flechas, dando su nombre común en inglés y entre paréntesis el nombre que se usará a lo largo de esta tesis.

\subsection{Superficies: simetría y sitios}

Las superficies se forman al cortar un cristal de una cierta simetría. El corte se realiza por medio de un plano, por lo que la simetría de la superficie formada puede ser cualitativamente distinta para cortes diferentes. La mayoría de los elementos de la tabla periódica forman cristales con simetría cúbica centrada en las caras (CCC) y en este trabajo nos restringiremos a este tipo de cristales. Los vectores unitarios del cristal CCC son $v_{1}=(0.5,0.5,0.0)$, $v_{2}=(0.0,0.5,0.5)$ y $v_{3}=(0.5,0.0,0.5)$ con la base de un solo átomo situado en $(x, y, z)=(0,0,0)$. Las tres caras superficiales más comunes (de índice bajo) son: (100), (110) y (111). Los tres índices entre paréntesis son llamados índices de Miller $[1,6]$. La cara (100) corresponde a un arreglo cuadrado, la (110) a un arreglo rectangular y la (111) a un arreglo hexagonal. La Figura 1.1 muestra un modelo finito para la superficie (111). Se pueden observar tres capas de superficie hacia abajo, con las flechas indicando los sitios de adsorción más comunes (de alta simetría). Una gran variedad de adsorbatos se localiza preferentemente en alguno(s) de 
estos sitios, por lo que vale la pena mencionarlos y asignar una letra por cada sitio, ya que se utilizarán recurrentemente a lo largo de esta tesis.

Un problema trascendental para la ciencia de superficies es encontrar los arreglos estables (no sólo el mínimo global) de una monocapa adsorbida sobre una superficie dada. Esta tarea es trivial para un solo átomo por celda unitaria. Sin embargo, se complica para $N>1$ átomos en la celda unitaria. Aunque el problema de encontrar el mínimo global de una celda con $N$ átomos para un sistema monocapa-superficie es típicamente más sencillo que en tres dimensiones (debido a que se puede considerar un sistema bi-dimensional), generalmente se quiere saber cual es el mínimo para una densidad superficial de adsorbato o grado de cobertura dado. Esto complica enormemente el problema de la búsqueda del mínimo, ya que a priori se desconoce la simetría de este.

Un modelo utilizado con frecuencia en sistemas fuertemente adsorbidos es el modelo de malla [6]. Se asume que los átomos adsorbidos pueden ocupar un solo sitio (el de mayor estabilidad) en la superficie, por lo que el problema se reduce a estudiar configuraciones de monocapas en una malla. Esta aproximación es válida solamente para los casos en los cuales el adsorbato tiene una probabilidad muy baja de encontrarse fuera del sitio de mínima energía. En estos casos, el arreglo de monocapa más estable será el que optimice la interacción lateral entre los átomos de adsorbato. Este modelo ha sido aplicado exitosamente a sistemas como metales alcalinos en superficies metálicas [7-9] y $\mathrm{Br}-\mathrm{Ag}(100)[10,11]$, entre otros.

Sin embargo, muchos sistemas reales no se pueden caracterizar por medio de este modelo y requieren el empleo de modelos contínuos para el potencial de superficie. En particular, éste es el caso de los halógenos en superficies metálicas que son los sistemas de interés en este trabajo. 

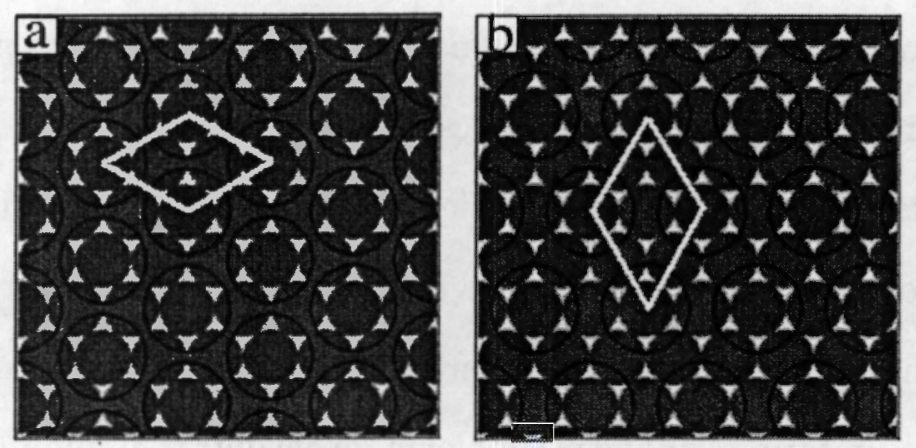

Figura 1.2: Modelos de estructuras $(\sqrt{3} \times \sqrt{3}) R 30^{\circ}$ y $(2 \times 2)$ sobre una superficie (111). Los círculos sólidos son los átomos del sustrato, mientras que los círculos negros más grandes son los átomos del adsorbato. La celda unitaria de la monocapa se indica por medio de un rombo blanco en ambos casos.

\subsection{Notación de Wood para estructuras en superficies}

En el caso más sencillo cuando una monocapa mantiene la simetría de la superficie, se puede utilizar la llamada notación de Wood [12] para describir la estructura de la monocapa. En este caso, la superficie se define por medio de dos vectores a y b y la monocapa por medio de $\mathbf{a}^{\prime} \mathbf{y} \mathbf{b}^{\prime}$. El ángulo entre $\mathbf{a}$ y $\mathbf{b}$ es el mismo que entre $\mathbf{a}^{\prime}$ y $\mathbf{b}^{\prime}$. Bajo esta condición, la notación de Wood es: $\left(\left|\mathbf{a}^{\prime}\right| /|\mathbf{a}| \times\left|\mathbf{b}^{\prime}\right| /|\mathbf{b}|\right) R \Theta^{\circ}$, donde $\Theta$ es el ángulo entre la celda unitaria de la monocapa y la del sustrato (superficie). En la Figura 1.2 se muestran ejemplos de las estructuras $(\sqrt{3} \times \sqrt{3}) R 30^{\circ}$ y $(2 \times 2)$ en una superficie con simetría (111).

\subsection{Potenciales de interacción}

El potencial de interacción en sistemas monocapa-superficie generalmente se separa en dos contribuciones: átomo adsorbido-superficie y átomo-átomo en la monocapa (interacción lateral). Para el caso de la interacción lateral por pares, la expresión general para este modo 

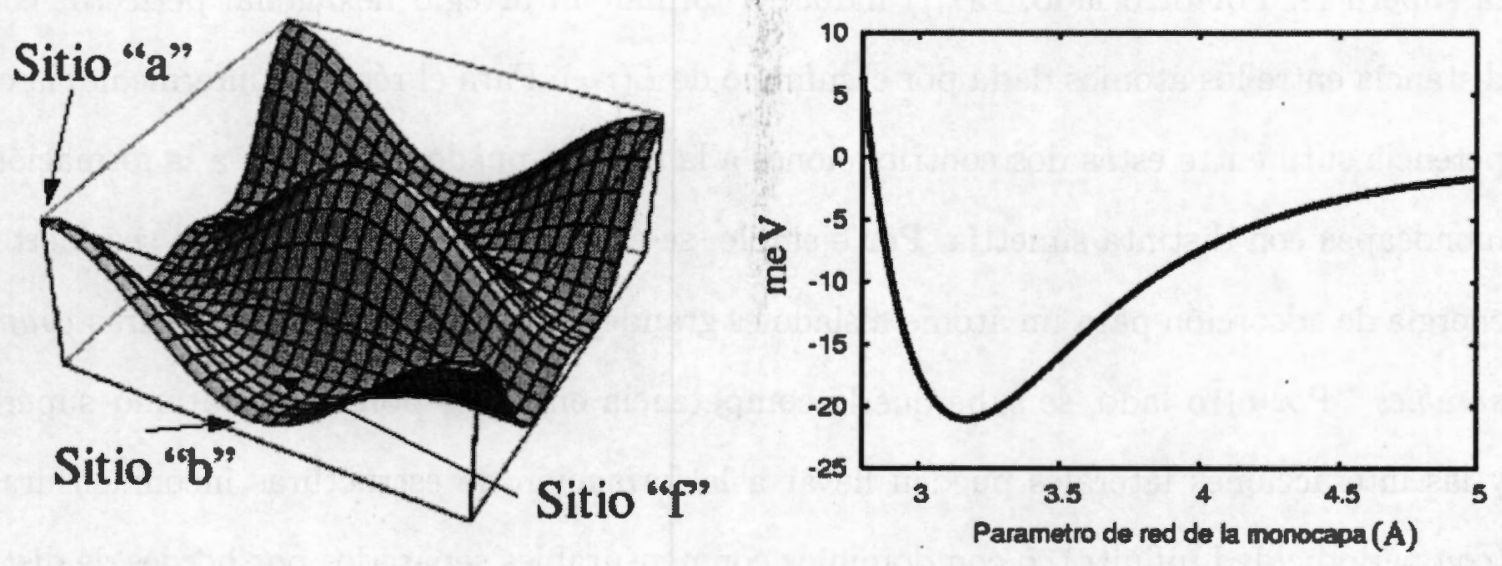

Figura 1.3: Ilustración de potenciales de interacción $V(x, y)$ (a la izquierda) y $L\left(r_{i j}\right)$ (a la derecha). Los sitios marcados sobre $V(x, y)$ se refieren a la Figura 1.1. En este caso, el sitio $a$ es el máximo global en la superficie (111). El potencial $L\left(r_{i j}\right)$ corresponde al modelo HFD-B2 [4] para una monocapa de xenon adsorbida sobre la superficie $\mathrm{Pt}(111)$ [incluyendo el efecto de la interacción repulsiva inducida por el sustrato [13]].

de partición de la energía de la monocapa es,

$$
E=\sum_{i}^{N}\left(V\left(x_{i}, y_{i}\right)+\sum_{j>i} L\left(r_{i j}\right)\right)
$$

donde $N$ es el número de átomos en la celda unitaria, $\left(x_{i}, y_{i}\right)$ es la posición del $i$-esimo átomo y $r_{i j}$ es la distancia entre los átomos $i$ y $j$. Las funciones $V\left(x_{i}, y_{i}\right)$ y $L\left(r_{i j}\right)$ corresponden a la interacción átomo-superficie e interacción lateral en la aproximación por pares, respectivamente. Un ejemplo específico de potenciales $V\left(x_{i}, y_{i}\right)$ y $L\left(r_{i j}\right)$ se ilustra en la Figura 1.3.

Hoy en día, se conocen muchos tipos de estructuras monocapa-superficie, caracterizadas por su simetría o el número de átomos por celda unitaria entre otros factores [1]. Las estructuras estables de una monocapa adsorbida en una superficie se pueden discutir en términos de la competencia entre los potenciales de interacción $V\left(x_{i}, y_{i}\right)$ y $L\left(r_{i j}\right)$. En un caso límite, el término $V\left(x_{i}, y_{i}\right)$ induce a la monocapa a ocupar los sitios de mínima energía en 
la superficie. Por otro lado, $L\left(r_{i j}\right)$ induce a formar un arreglo hexagonal perfecto ${ }^{\mathrm{a}}$ con la distancia entre los átomos dada por el mínimo de $L\left(r_{i j}\right)$. Para el régimen intermedio, la competencia sutíl entre estas dos contribuciones a la energía puede dar origen a la formación de monocapas con distinta simetría. Por ejemplo, se espera que cuando $V\left(x_{i}, y_{i}\right)$ sea fuerte (la energía de adsorción para un átomo aislado es grande), el sistema forme estructuras conmensurables. ${ }^{b}$ Por otro lado, se sabe que la competencia entre los potenciales átomo-superficie y las interacciones laterales pueden llevar a la formación de estructuras inconmensurables (con periodicidad infinita), o con dominios conmensurables separados por bordes de distinta simetría. En esta discusión no se puede abarcar todo el universo de posibilidades para las estructuras monocapa-superficie encontradas en los estudios teóricos y experimentales hasta la fecha.

En general, la función $L\left(r_{i j}\right)$ puede ser de una naturaleza muy compleja y es difícil tener expresiones analíticas para ésta. Actualmente $L\left(r_{i j}\right)$ se conoce con cierta exactitud solamente para pocos sistemas, como los gases nobles en $\mathrm{Pt}(111)$ [4]. La complejidad de esta función radica en el hecho de que en la adsorción sobre una superficie están presentes efectos electrónicos mediados por el sustrato. En general, las expresiones analíticas para este tipo de interacciones no se conocen, aunque algunos casos límite para fisisorción sean conocidos [13].

Las expresiones que describen la interacción entre un átomo adsorbido y una superficie pueden ser muy complejos también, pero es importante notar que hay una forma rigurosa de describir la función $V\left(x_{i}, y_{i}\right)$ con la precisión deseada. Esto se puede hacer mediante el empleo de la expansión de Fourier de $V\left(x_{i}, y_{i}\right)$ con la simetría de la superficie correspondiente [14].

${ }^{a}$ El mínimo global para partículas en una caja en dos dimensiones interactuando por medio de un potencial isotrópico y con un solo mínimo tiene simetría hexagonal.

bUna estructura conmensurable se define como aquella que tiene una celda unitaria finita sobre el sustrato y posee la misma simetría. Por otro lado, en términos energéticos, una estructura conmensurable se caracteriza por tener un porcentaje de átomos en los sitios de mínima energía, por lo que se cree que su formación se puede favorecer en comparación con estructuras inconmensurables - las que poseen una celda unitaria infinita sobre el sustrato. 
En este caso, $V\left(x_{i}, y_{i}\right)$ se escribe como:

$$
V(\mathbf{r})=V_{0}(z)+\sum_{G} V_{G}(z) \exp (-\imath \mathrm{Gr})
$$

donde $\mathbf{r}$ es el vector en el espacio cartesiano, $\mathbf{G}$ son los vectores del espacio recíproco (de Fourier), $V_{0}(z)$ es el potencial lateral promedio y $V_{G}(z)$ son los coeficientes de Fourier. En general, los coeficientes $V_{G}(z)$ dependen de la coordenada vertical $z$, aunque en esta tesis se asume que cada $V_{G}$ se toma para el valor de equilibrio de $z$ para el sitio correspondiente, ${ }^{\text {a }}$ formando así una estructura en dos dimensiones, aunque implicitamente contiene la corrugación geométrica por tomar el valor de $V_{G}(z)$ para $z$ 's de equilibrio. Se sabe que en los casos sencillos de fisisorción, donde las interacciones entre el átomo adsorbido y la superficie son de tipo Lennard-Jones, la expansión de la Ecuación 1.2 converge rápidamente y unos cuantos términos de la misma son suficientes para obtener resultados confiables [4]. Sin embargo, en la literatura actual no existe un estudio de esta expansíon para casos más complejos y en general, su comportamiento es desconocido para potenciales que no sean de tipo Lennard-Jones. Por otro lado, los coeficientes $V_{G}$ se pueden obtener desde cálculos de primeros principios (p. ej. basados en la TFD), logrando una descripción adecuada del potencial de interacción átomo-superficie para una variedad de sistemas. Aunque la expansión de Fourier proporciona una forma matemática exacta de representar cualquier función con la simetría correcta de una superficie, en muchos casos no es la mejor forma de describir la interacción átomo-superficie por lo complejo que resulta obtener los coeficientes $V_{G}$ para sistemas reales. Debido a esto, se han propuesto distintas formas sencillas de describir estas interacciones. Una de ellas, desarrollada por el autor de esta tesis en conjunto con Nikola Batina, está basada en empaquetamiento de esferas, y se llama USP por sus siglas en

\footnotetext{
'La función de interacción átomo-superficie se puede escribir como $E(x, y, z)$. Sin embargo, para cada $(x, y)$ fijo, la función $E(x, y, z)$ tiene un mínimo en el caso de adsorción exotérmica. Es por eso que la interacción átomo-superficie se puede mapear a una función que solamente depende de $x$ y $y$ explicitamente: $P(x, y)=\min _{z} E(x, y, z)$.
} 

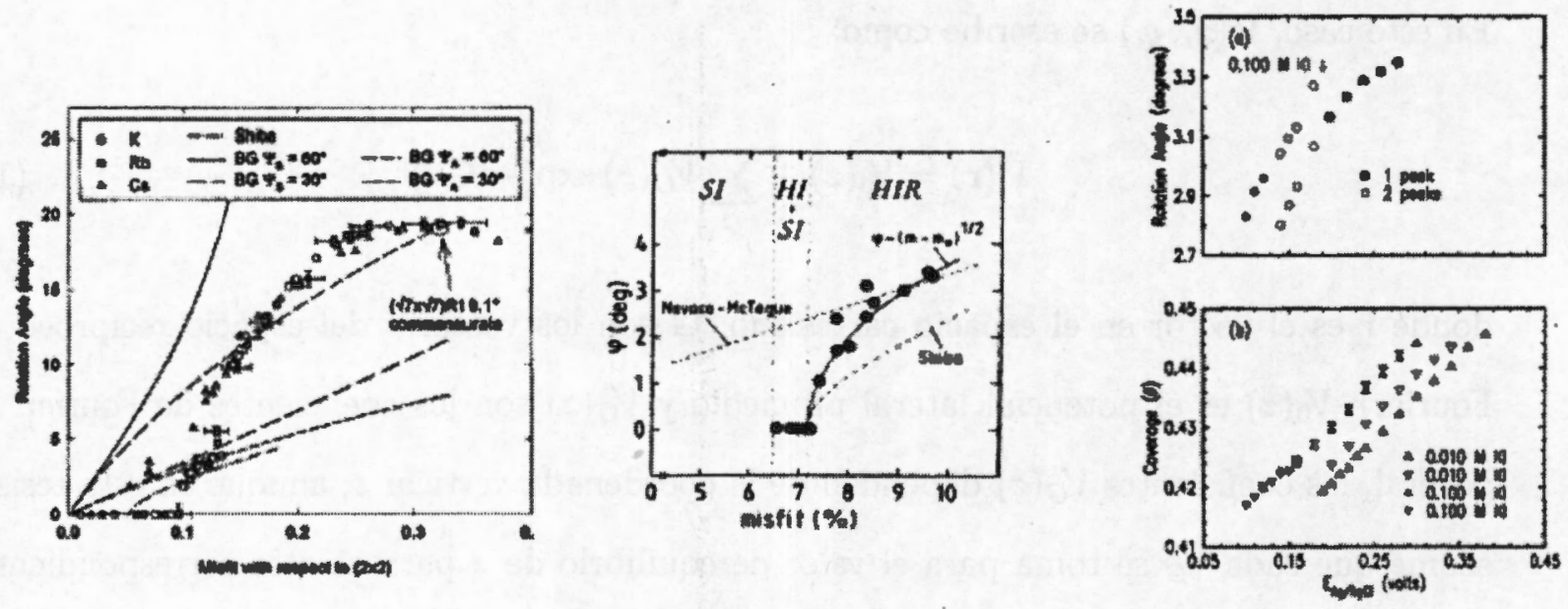

Figura 1.4: Ejemplos de comportamiento epitaxial (ángulo de rotación como función de la distancia entre átomos en la monocapa) para $\mathrm{K}, \mathrm{Rb}$ y $\mathrm{Cs}$ en $\mathrm{Ag}$ (111) [a la izquierda], $\mathrm{Xe}-\mathrm{Pt}$ (111) [al centro] y I-Au(111) [a la derecha]. Las figuras fueron tomadas de las referencias [19-21], respectivamente.

inglés [15-18].

Por lo dicho anteriormente, se puede concluir que no existe un algoritmo general que permita extraer los potenciales desde cálculos de primeros principios. Esta tesis se va a concentrar fundamentalmente en el análisis del potencial átomo-superficie y su efecto sobre las estructuras ordenadas monocapa-superficie.

\subsection{Modelos para comportamiento epitaxial}

La técnica experimental de difracción de electrones de baja energía (LEED por sus siglas en inglés) ha sido la más frecuentemente utilizada para estudiar la estructura de monocapas en superficies $[1,22,23]$. Esta técnica permite deducir el parámetro de red de la monocapa y su ángulo de rotación con respecto al sustrato. Variando la temperatura, la presión del gas de los adsorbatos o el potencial de la celda (en ambiente electroquímico) se puede lograr una variación continua del grado de cobertura del adsorbato en la superficie. De esta forma, se puede obtener un comportamiento del ángulo de rotación como función de la distancia entre 
los adsorbatos en la monocapa. Esto da origen al llamado comportamiento epitaxial ${ }^{\mathrm{a}}$ de la monocapa sobre una superficie. Se pueden distinguir muchos tipos de crecimiento epitaxial en monocapas adsorbidas sobre superficies ordenadas. Dichos arreglos están caracterizados por rotaciones o traslaciones colectivas de los átomos en la monocapa. Los comportamientos epitaxiales han generado mucho interés desde el punto de vista teórico y experimental $[1,4,6]$. Algunos ejemplos de comportamiento epitaxial se presentan en la Figura 1.4 para los casos de metales alcalinos en $\mathrm{Ag}(111), \mathrm{Xe}-\mathrm{Pt}(111)$ y $\mathrm{I}-\mathrm{Au}(111)$. La descripción detallada de las estructuras de monocapas adsorbidas y las transiciones de fase entre ellas ha sido uno de los temas importantes en la literatura, y las monocapas cuadradas sobre sustratos con simetría cuadrada o rectangular han sido de las más estudiadas por métodos teóricos [24-26]. Se ha propuesto una forma sencilla para clasificar las estructuras de monocapas con respecto al sustrato, por medio de la matriz que asocia los vectores de la celda de la monocapa con los vectores de la celda de la superficie $[27,28]$. En este trabajo nuestro mayor interés es estudiar monocapas en la cara (111) de superficies metálicas. Muchos sistemas experimentales reales forman monocapas hexagonales en superficies (111): gases nobles en superficies metálicas y grafito, halógenos en superficies metálicas, metales alcalinos en superficies metálicas, entre otros. Usualmente, estos sistemas presentan comportamientos epitaxiales interesantes y varios modelos se han propuesto para describirlos.

El modelo de Novaco-McTague es uno de los más conocidos [29,30]. En este caso, la monocapa es descrita por medio de constantes elásticas y se demuestra que el estado basal de la monocapa adsorbida se puede describir con un ángulo de rotación asimétrico con respecto al sustrato. La interacción de la capa adsorbida con el sustrato no se toma en cuenta y la rotación epitaxial es controlada por la interacción entre los átomos de la monocapa exclusiva-

\footnotetext{
"El término epitaxial proviene del griego epi "sobre" y taxis "de forma ordenada". En este caso referimos a esta palabra para indicar el tipo de orden en la formación de monocapas sobre una superficie. En este trabajo particular, este orden puede ser definido mediante una distancia interatómica entre los átomos en la monocapa y el ángulo de rotación de esta con respecto al sustrato (superficie).
} 
mente. Otro modelo distinto ha sido propuesto por Doering [31], el cual usa consideraciones de simetría para explicar el comportamiento rotacional de la monocapa adsorbida. Grey y Bohr $[32,33]$ usaron un modelo de interacción de átomo-superficie con un solo término de Fourier, y propusieron que el efecto de tamaño finito debido a las terrazas de la superficie contribuye significativamente a la rotación de la monocapa. Por otro lado, Shiba $[34,35]$ propuso una teoría basada en los bordes entre dominios (conmensurables) para explicar el comportamiento de los gases nobles en superficies metálicas. Todos estos modelos obtienen comportamientos cualitativamente distintos para el ángulo de rotación de la monocapa. Aún tomando en cuenta la variedad de modelos propuestos, muchos sistemas experimentales no se pueden describir por ninguno de estos. Un ejemplo claro son los metales alcalinos en la superficie $\mathrm{Ag}(111)$ [19]. El comportamiento del ángulo de rotación de las monocapas hexagonales de $\mathrm{K}, \mathrm{Rb}$ y Cs adsorbidos sobre $\mathrm{Ag}(111)$ es diferente a cualquiera de los modelos descritos antes. Otro ejemplo son las monocapas de gases nobles en la superficie $\mathrm{Ag}(111)$ [36], donde se ha propuesto que la interacción sustrato-adsorbato juega un rol importante. El comportamiento cuantitativo de las monocapas de halógenos en las superficies $\mathrm{Pt}(111), \mathrm{Au}(111)$ y $\mathrm{Ag}(111)$ tampoco se ha explicado desde el punto de vista teórico [21,37-39]. Un aspecto común para todos los modelos previos del comportamiento epitaxial rotacional de monocapas adsorbidas, es que la interacción de los átomos adsorbidos con el sustrato se asume como muy débil o descrita por medio de un solo término de la expansión de Fourier. Aunque esta aproximación ha demostrado ser válida para interacciones simples por pares como es el caso de algunos gases nobles en grafito y Xe-Pt(111) [40-42], para casos más complejos, la descripción del potencial de interacción átomo-superficie requiere términos de Fourier de orden superior [43].

Como se puede ver de la discusión del párrafo anterior, cada uno de los modelos para comportamiento epitaxial propuestos hasta la fecha cubren solamente una parte del universo posible de las interacciones $V(x, y)$ y $L\left(r_{i j}\right)$. Por ejemplo, el modelo de Novaco-McTague [29, 
30] desprecia la interacción átomo-superficie $V(x, y)$ y los efectos anharmónicos en $L\left(r_{i j}\right)$. Los modelos basados en la geometría [31-33] ignoran el efecto de las interacciones laterales y suponen un arreglo rígido de la monocapa. Debido a que el potencial de interacción átomosuperficie no se ha analizado a detalle en trabajos previos (su descripción se ha limitado al primer término de Fourier solamente), una parte importante de esta tesis versará sobre un análisis detallado del efecto del potencial átomo-superficie sobre estructuras de monocapas adsorbidas. Esto permitirá resolver el problema de la monocapa rígida de una manera general. 


\section{Capítulo 2}

\section{Monocapa rígida hexagonal}

En este capítulo se analiza a detalle el caso de una monocapa rígida hexagonal adsorbida sobre una superficie (111). Primero se analiza la simetría de capas hexagonales y los "números mágicos" de átomos por celda unitaria para estas capas. Despues se discute la validez de esta aproximación en el caso de sistemas reales. Posteriormente, se hace una formulación analítica para describir estados basales (mínimos) de una monocapa rígida hexagonal infinita adsorbida en una superficie (111) lo que proporciona una identificación de estructuras conmensurables que se asocian directamente a distintos términos de la expansión de Fourier del potencial atomo-superficie.

\subsection{Monocapas hexagonales: consideraciones de si- metría}

En esta sección se presentará una clasificación de las monocapas hexagonales basada en el número de átomos en la celda primitiva; también se ilustran algunas propiedades de esta estructura algebráica y la forma de generar las coordenadas de los átomos para una monocapa

aparte de este capítulo esta basado en el artículo Analytic theory of hexagonal monolayer interacting with hexagonal substrate, A. Tkatchenko, Phys. Rev. B 74, 035428 (2006). 
hexagonal en una superficie (111). Esto dará una herramienta poderosa para clasificar los arreglos conmensurables de monocapas y realizar una construcción eficiente de parámetros de malla y coordenadas atómicas para cálculos de TFD posteriores.

La celda primitiva de una superficie (111) con los átomos de diámetro atómico $d=1$ (en unidades del parámetro de red de la superficie) es generada por dos vectores: $v_{1}=(1,0)$ y $v_{2}=(0.5,0.5 \sqrt{3})$ con un ángulo de $60^{\circ}$ entre ellos. La distancia desde el origen puesto en el centro de algún átomo en $(x, y)=(0,0)$ a cualquier otro átomo en la malla hexagonal es dada por $D=\sqrt{a^{2}+b^{2}+a b}$, donde $a$ y $b$ son enteros. Es importante recordar en este punto que la malla hexagonal en espacio recíproco es otra malla hexagonal rotada por $30^{\circ}$. Esto da origen a que la longitud de los vectores en espacio recíproco se puede escribir como $D G_{0}$, donde $G_{0}=\frac{4 \pi}{\sqrt{3}}$ es la longitud del vector recíproco más pequeño. $D^{2}$ es un entero y forma la llamada secuencia de números hexagonales [44]. Cualquier celda no-primitiva de la malla hexagonal se puede obtener como una combinación lineal de los vectores primitivos: $V_{1}=(a+0.5 b,-0.5 \sqrt{3} b), V_{2}=(0.5 a+b, 0.5 \sqrt{3} a)$, donde $a$ y $b$ son enteros. Es interesante notar que el número de átomos en una celda de estas características está dado también por $N=a^{2}+b^{2}+a b$. Para mostrar esto, nótese que el área de una celda hexagonal esta contenida en un rombo con el área igual a $A_{\text {rombo }}=0.5 \sqrt{3}\left(a^{2}+b^{2}+a b\right)$. Por otro lado, un átomo ocupa el área de un círculo con $d=1$, dada por $\pi / 4$. La densidad de un arreglo de círculos hexagonal es $\sqrt{3} \pi / 6$, lo que da un área efectiva ocupada por círculos de $A_{\text {circ }}=0.5 \sqrt{3}$. Dividiendo $A_{\text {rombo }}$ por $A_{\text {circ }}$, recuperamos la secuencia de números hexagonales de nuevo. Esto significa que una celda hexagonal con $2 \circ 5$ partículas no puede existir. Los primeros términos (no cero) de la secuencia son $1,3,4,7$, etc.

Es importante discutir sobre algunas propiedades matemáticas de la secuencia de números hexagonales, ya que se usarán más adelante. La ecuación

$$
m=a^{2}+b^{2}+a b
$$


es llamada una forma cuadrática binaria o una ecuación Diofantina [45] y éstas se estudian en la teoría de números. Llamaremos $\mathcal{H}$ el conjunto de todas las $m$ (ordenadas en orden ascendente y excluyendo cero). El primer hecho importante es que, hasta la fecha, no existe una fórmula analítica estableciendo una correspondencia uno a uno entre los elementos de $\mathcal{H}$ y los enteros positivos $\mathbb{N}^{+}$. Sin embargo, existe una forma sencilla de encontrar el número de soluciones (o la degeneración en terminología física) a la Ecuación 2.1 para un valor particular de $m$. Está dado por $6 E(m)[46]$, donde $E(m)$ es la diferencia entre los divisores de $m$ de la forma $3 l+1$ y de la forma $3 l+2$. Cuando $E(m) \leq 0$ (v.g., para $m=2,5, \ldots$ ), $m$ no pertenece a la secuencia de números hexagonales, mientras que para $E(m)>0$, hay un número finito de soluciones a la Ecuación 2.1. Más aún, $E(m)$ no es una función acotada, lo que se puede mostrar para el caso de $m=p^{n}$, donde $p$ es un número primo $(p \neq 3)$ que pertenece a la secuencia de números hexagonales y $n$ es un entero. En este caso, $E(m)=n+1$. En términos geométricos esto significa que puede haber un número arbitrariamente grande de átomos con la distancia $D=\sqrt{p^{n}}$ desde el origen en la malla hexagonal. Por ejemplo, $E(m)=1$ para $m=1,3,4$, pero $E(m)=2$ para $m=7$. Este hecho es muy importante para la expansión de Fourier del potencial átomo-superficie ya que $6 E(m)$ es exactamente el número de vectores recíprocos de longitud $D=\sqrt{m}$. Así mismo, podemos ver que el conjunto $\mathcal{H}$ es cerrado bajo multiplicación, i.e. si $m_{1}, m_{2} \in \mathcal{H} \Rightarrow m_{1} m_{2} \in \mathcal{H}$. Necesitamos demostrar que $\left(a_{1}^{2}+b_{1}^{2}+a_{1} b_{1}\right)\left(a_{2}^{2}+b_{2}^{2}+a_{2} b_{2}\right)=\left(a_{3}^{2}+b_{3}^{2}+a_{3} b_{3}\right), \forall a_{i} \in \mathbb{N}^{+}$. De hecho, esta identidad se cumple para $a_{3}=a_{1} a_{2}-b_{1} b_{2}$ y $b_{3}=a_{1} b_{2}+b_{1} b_{2}+b_{1} a_{2}$. La propiedad de cerradura se va a utilizar más adelante para generar estructuras conmensurables con un número de átomos progresivamente mayor.

Dada la secuencia de números hexagonales, cualquier estructura conmensurable de una monocapa sobre una superficie se puede construir con $N_{s u s}=n^{2}+k^{2}+n k$ y $N_{a d s}=a^{2}+b^{2}+a b$, donde $N_{\text {sus }}$ es el número de átomos del sustrato en la celda y $N_{\text {ads }}$ corresponde al número de átomos del adsorbato (monocapa); $n, k$ y $a, b$ son enteros. Mediante esta clasificación, 
una estructura conmensurable con grado de cobertura $\theta=\frac{N_{a d e}}{N_{s u s}}$ está definida excepto por la posición del centro de masa (grados de libertad traslacionales). La distancia interatómica (el parámetro de red) de la monocapa se puede calcular como $R=\sqrt{\frac{N_{\text {sas }}}{N_{\text {ade }}}}$. Adicionalmente, para ajustar la monocapa con el sustrato, ésta tiene que ser rotada por un cierto angulo $\alpha$, el cual no es único para $N_{\text {ads }}$ y $N_{\text {sus }}$ dados. El ángulo $\alpha$ se puede calcular imponiendo la restricción de que un átomo de la monocapa tiene que coincidir con un átomo del sustrato. Una vez que $R$ y $\alpha$ de la monocapa estan determinados, se puede escribir un algoritmo sencillo para generar las posiciones de los átomos en las capas de adsorbato y sustrato, como sigue:

(1) Generar la secuencia de números hexagonales de la Ecuación 2.1, obteniendo todas las $m$ con sus valores respectivos de $a$ y $b$.

(2) Utilizar paso (1) para obtener una combinación de $n$ y $k$ que genera $N_{s u s}$ y, como consecuencia, los vectores de la celda unitaria $V_{1}=(n+0,5 k,-0.5 \sqrt{3} k), V_{2}=(0.5 n+$ $k, 0.5 \sqrt{3} k)$.

(3) Calcular todas las combinaciones lineales de $v_{1}$ y $v_{2}: V_{s u s}=c v_{1}+d v_{2}$ para $-N \leq$ $c, d \leq N$, con $N$ grande; $v_{1}$ and $v_{2}$ son los vectores primitivos de la cara (111).

(4) Para cada $V_{\text {sus }}$ revisar si las coordenadas $(x, y)$ caen dentro de la celda unitaria. Descartar todos los $V_{\text {sus }}$ que no cumplen con esta condición.

(5) Generar las posiciones de los átomos en la monocapa, donde cada $V_{a d s}$ está dada por

$$
\begin{aligned}
V_{a d s}= & {[(c+0.5 d) R \cos \alpha-0.5 \sqrt{3} d R \sin \alpha,} \\
& 0.5 \sqrt{3} d R \cos \alpha+(c+0.5 d) R \sin \alpha],
\end{aligned}
$$

donde $c$ y $d$ son enteros.

(6) Repetir paso (4) para cada $V_{\text {ads }}$.

Los valores explicitos de $a$ y $b$ que generan $N_{a d s}$ solo són necesarios para obtener $\alpha$ (esto se mostrará más adelante). 
La descripción matemática desarrollada en esta sección se utilizará a lo largo de la tesis (en mayor parte más adelante en este capítulo) para diseñar una forma de clasificar monocapas rígidas hexagonales y encontrar mínimos descritos por $R$ y $\alpha$. Por otro lado, el algoritmo de generación de posiciones atómicas de estructuras conmensurables se utilizará para generar las geometrías iniciales para cálculos de TFD, aún sin mencionarlo explícitamente.

\subsection{Aproximación de monocapa rígida}

Es interesante analizar el caso cuando la función de la interacción lateral $L\left(r_{i j}\right)$ (ver Ecuación 1.1) en la monocapa adsorbida es fuertemente repulsiva. En este caso, se favorece la formación de monocapas hexagonales rígidas, por lo que la monocapa puede ser descrita por medio de un número reducido de variables (parámetros de orden).

Para verificar la validez de esta aproximación rígida para sistemas sustrato-adsorbato reales, vamos a analizar las magnitudes de interacciones átomo-superficie y átomo-átomo en la monocapa en tres casos: I-Pt(111) [47,48], Xe-Pt(111) $[49,50]$ y Xe-Pd(111) [50], para los cuales hay datos disponibles de cálculos de TFD para distintos sitios de adsorción. Esto nos permite calcular los primeros coeficientes de la serie de Fourier para el potencial átomosuperficie.

Se escogió el sistema I-Pt(111) como un ejemplo de adsorción de halógenos en superficies metálicas (quimisorción) y éste será comparado con sistemas Xe-Pt(111) y Xe-Pd(111). Estos últimos son ejemplos claros de fisisorción, que además han sido estudiados con cierto detalle y existe información confiable sobre las interacciones [51,52].

Primero, estudiamos la validez del modelo de un sólo término de Fourier para representar la interacción átomo-superficie para los tres sistemas. Se utiliza el cociente $\left(E_{a}-E_{b}\right) /\left(E_{a}-\right.$ $E_{f}$ ), donde los subíndices se refieren a los sitios de la Figura 1.1. Este cociente ubica la posición energética del sitio $b$ dentro del rango completo $E_{a}-E_{f}$ y proporciona una forma 
sencilla de evaluar distintos modelos de interacción átomo-superficie con energías de tres sitios de alta simetría. Para el caso del modelo con un sólo término de Fourier, este cociente debería ser igual a 0.89 . Vea la Figura 1.3 para la ilustración del potencial átomo-superficie con un sólo término de Fourier. Tomaremos datos de cálculos TFD para ajustar los primeros tres coeficientes de la serie de Fourier para I-Pt(111) [47], Xe-Pt(111) [49] y Xe-Pd(111) [50]. Para el caso de I-Pt(111) [47] el cociente es 0.78, para Xe-Pt(111) [49] es 0.92 y para Xe$\operatorname{Pd}(111)[50]$ es 0.76 . De aquí se observa que, mientras que la expansión de Fourier cortada después del primer término puede ser suficiente para el caso de $\mathrm{Xe}-\mathrm{Pt}(111)$, lo mismo no se puede concluir para I-Pt(111) y Xe-Pd(111). Por otro lado, el segundo coeficiente de Fourier en el caso de I-Pt(111) es $V_{\sqrt{3} G_{0}}=-0.24 V_{G_{0}}$, para $\mathrm{Xe}-\mathrm{Pt}(111)$ es $V_{\sqrt{3} G_{0}}=0.05 V_{G_{0}}$ y para Xe-Pd(111) $V_{\sqrt{3} G_{0}}=-0.08 V_{G_{0}}$. De este sencillo análisis se podría concluir que la expansión de Fourier muestra una convergencia más rápida en el caso de sistemas de fisisorción. Sin embargo, se requiere un estudio sistemático para poder confirmar este hecho.

Los estudios experimentales indican que las estructuras conmensurables $(\sqrt{3} \times \sqrt{3}) R 30^{\circ}$ y $(\sqrt{7} \times \sqrt{7}) R 19.11^{\circ}$ existen para I-Pt(111) [37] y para Xe-Pd(111) [53-55], mientras que solamente $(\sqrt{3} \times \sqrt{3}) R 30^{\circ}$ se encontró en el caso de $\mathrm{Xe}-\mathrm{Pt}(111)$ [56]. Adicionalmente, la estructura conmensurable $(3 \times 3)$ existe para I-Pt(111) [37] y $(\sqrt{19} \times \sqrt{19}) R 23.4^{\circ}$ existe para $\mathrm{Xe}-\mathrm{Pd}(111)[55]$.

En la siguiente sección se demostrará que el primer término de Fourier (el que generalmente contribuye más al potencial átomo-superficie) se cancela en el caso de estructuras rígidas hexagonales con más de un átomo por celda unitaria. Esto indica que en una vecindad cercana a los arreglos hexagonales rígidos este término tiene una contribución pequeña. Por el contrario, el primer término se conserva completamente para la estructura $(\sqrt{3} \times \sqrt{3}) R 30^{\circ}$ con un solo átomo por celda unitaria y el segundo término se conserva para la estructura $(\sqrt{7} \times \sqrt{7}) R 19.11^{\circ}$ con tres átomos por celda unitaria. Considerando que estos arreglos se han observado en experimentos para I-Pt(111) y Xe-Pd(111), podemos utilizar la informa- 
ción sobre las magnitudes de interacciones átomo-superficie y átomo-átomo en la monocapa para estimar condiciones necesarias para la validez de la aproximación hexagonal rígida en sistemas reales. Utilizaremos el modelo HFD-B2 [52] con la dispersión de McLachlan [13] para describir las interacciones laterales en el sistema $\mathrm{Xe}-\mathrm{Pd}(111)$ y un modelo de repulsión exponencial ajustado a cálculos de TFD para I-Pt(111) [57]. En la Figura 1.3 se muestra el modelo del potencial HFD-B2 para el caso de xenon adsorbido en la superficie $\mathrm{Pt}(111)$.

El mínimo de potencial HFD-B2 con dispersión de McLachlan para Xe-Xe esta situado en $1.59 l$, donde $l$ es la constante de red del sustrato [ $l=2.775 \AA$ para el caso de $\operatorname{Pt}(111)]$. La distancia interatómica en la estructura $(\sqrt{3} \times \sqrt{3}) R 30^{\circ}$ es $\sqrt{3} l$ con el gradiente del potencial de $21 \mathrm{meVl}^{-1}$ en ese punto (parte atractiva de la interacción), mientras que para $(\sqrt{7} \times \sqrt{7}) R 19.11^{\circ}$ la distancia es $1.53 l$ con gradiente de $-32 \mathrm{meVl}^{-1}$ y está situada sobre la parte repulsiva de la curva de interacción. La energía de interacción lateral total para la estructura rígida $(\sqrt{7} \times \sqrt{7}) R 19.11^{\circ}$ es $-58.7 \mathrm{meV}$, mientras que el segundo coeficiente de Fourier es de $0.83 \mathrm{meV}$. En el caso del sistema I-Pt(111), el gradiente para la estructura $(\sqrt{7} \times \sqrt{7}) R 19.11^{\circ}$ es alrededor de $-301 \mathrm{meVl}^{-1}$ y la energía de interacción total es de 205 $\mathrm{meV}$ [48], mientras que el segundo coeficiente de Fourier es de $-23.22 \mathrm{meV}$. Se puede ver que el cociente $\left|V_{\sqrt{3} G_{0}} / \Delta E_{\text {lateral }}\right|$ para $(\sqrt{7} \times \sqrt{7}) R 19.11^{\circ}$ varía de 0.03 para $\mathrm{Xe}-\mathrm{Pd}(111)$ a 0.08 para I-Pt(111). Considerando que las estructuras obtenidas experimentalmente son rígidas, la aproximación utilizada en el presente trabajo sería exacta para estas magnitudes de interacción.

\subsection{Teoría analítica de monocapa rígida hexagonal in- finita}

En esta sección se desarrolla una teoría analítica de una monocapa rígida hexagonal que interactúa con una superficie ordenada (111). La interacción átomo-superficie esta descrita 
por medio de una expansión de Fourier con un número variable de términos, hasta un número posiblemente infinito.

La interacción de un átomo adsorbido con la superficie es descrita por la serie de Fourier $[4$, 14]:

$$
V(\mathbf{r})=V_{0}+\sum_{G} V_{G} \exp (-\imath \mathbf{G r})
$$

donde $\mathbf{r}$ es un vector en el espacio real, $\mathbf{G}$ son los vectores de la celda en el espacio recíproco (de Fourier) y los $V_{G}$ son los coeficientes de Fourier. Usualmente, para una representación del potencial en tres dimensiones (incluido $z$ ), los coeficientes $V_{G}$ dependen de la coordenada vertical $z$ del átomo arriba de la superficie, pero como veremos más adelante, los resultados de esta teoría no dependen de la forma de los $V_{G}$. Cuando es necesario, en este trabajo los valores de $V_{G}$ son tomados para la distancia $z$ de equilibrio. Es importante notar que la diferencia entre distintos sistemas sustrato-adsorbato está "codificada" en los coeficientes de Fourier.

En el caso de interacciones por pares sencillas entre el átomo adsorbido y los átomos de la superficie, los coeficientes decaen rápidamente [14], y pocos términos pueden dar una descripción precisa de la interacción. Por otro lado, para potenciales de interacción más complejos, el comportamiento de los coeficientes de la serie no es conocido y los cálculos basados en Teoría de Funcionales de la Densidad (TFD) pueden ser empleados para obtenerlos $[43,58]$. En esta sección queremos estudiar la respuesta de la monocapa hexagonal rígida infinita a los cambios en $V(\mathbf{r})$. Una monocapa de estas características puede ser descrita por medio de cuatro variables: distancia interatómica $(R)$, ángulo de rotación $(\alpha)$ y posición sobre el sustrato $\left(x_{0}, y_{0}\right)$. Las coordenadas de cada átomo en una monocapa hexagonal sin rotar se obtienen como una combinación lineal de dos vectores $l_{1}=R(2,0)$ y $l_{2}=R(1, \sqrt{3})$. Si permitimos la rotación de la monocapa por un ángulo $\alpha$, la posición de cualquier átomo se puede 
escribir como:

$$
\mathbf{r}=\mathbf{r}_{\mathbf{0}}+M_{\alpha}\left(a l_{1}+b l_{2}\right)
$$

donde

$$
M_{\alpha}=\left(\begin{array}{cc}
\cos \alpha & -\sin \alpha \\
\sin \alpha & \cos \alpha
\end{array}\right)
$$

Expandiendo las dos últimas ecuaciones da como resultado:

$$
\begin{array}{r}
\mathbf{r}=\left(x_{0}+R(2 a+b) \cos \alpha-\sqrt{3} R b \sin \alpha,\right. \\
\left.y_{0}+\sqrt{3} R b \cos \alpha+R(2 a+b) \sin \alpha\right)
\end{array}
$$

donde $a$ y $b$ son enteros. Poniendo explícitamente la dependencia de $V(\mathbf{r})$ en la Ecuación 2.3 da,

$$
V(\mathrm{r})=V\left(a, b, R, \alpha, x_{0}, y_{0}\right)=V_{0}+\sum_{G} V_{G} \exp (-\imath \mathbf{G r})
$$

Así mismo, se puede demostrar que $\exp (-\imath \mathrm{Gr})$ puede ser escrito de la siguiente manera:

$$
\begin{aligned}
\exp (-\imath \mathbf{G r})= & \exp \left(-\imath\left(\mathbf{G r}_{0}+C_{a}(R, \alpha, \mathbf{G}) a+C_{b}(R, \alpha, \mathbf{G}) b\right)\right) \\
= & \exp \left(-\imath \mathbf{G r}_{0}\right) \times \exp \left(-\imath C_{a}(R, \alpha, \mathbf{G}) a\right) \\
& \times \exp \left(-\imath C_{b}(R, \alpha, \mathbf{G}) b\right)
\end{aligned}
$$

donde $\mathrm{r}_{0}=\left(x_{0}, y_{0}\right)$, mientras que $C_{a}$ y $C_{b}$ son los términos asociados con $a$ ó $b$, respectivar mente.

Se quiere encontrar el conjunto de $\left(R, \alpha, x_{0}, y_{0}\right)$ para el cual la energía total de la monocapa,

$$
E\left(R, \alpha, x_{0}, y_{0}\right)=\sum_{a=-\infty}^{\infty} \sum_{b=-\infty}^{\infty} V\left(a, b, R, \alpha, x_{0}, y_{0}\right),
$$

se minimiza. En realidad, no estamos interesados en la energía de la monocapa infinita, ya 
que ésta tiene un valor infinito. La cantidad que es importante en este caso es la energía por átomo adsorbido. Para lograr esto, se reescribe la Ecuación 2.9 como un límite infinito de una sumatoria parcial:

$$
\begin{aligned}
E_{\text {atom }}\left(R, \alpha, x_{0}, y_{0}\right)= & \lim _{m \rightarrow \infty} \frac{1}{m^{2}} \sum_{k=0}^{m} \sum_{a=-k}^{k} V_{a}\left(a, R, \alpha, x_{0}, y_{0}\right) \\
& \times \lim _{m \rightarrow \infty} \frac{1}{m^{2}} \sum_{k=0}^{m} \sum_{b=-k}^{k} V_{b}\left(b, R, \alpha, x_{0}, y_{0}\right)
\end{aligned}
$$

donde $m^{2}$ es el número de términos en las sumatorias dobles, lo que proviene de resolver una ecuación recursiva. La última ecuación no depende explicitamente de $N$ - el número de átomos en la celda unitaria de la monocapa, sin embargo más adelante se mostrará que $N$ es una función de $R$ y $\alpha$, con lo que podemos recuperar la idea de energía como función de $R$ y $\alpha$ exclusivamente.

Para encontrar los mínimos de la Ecuación 2.10, se utiliza la siguiente identidad,

$$
\lim _{m \rightarrow \infty} \frac{1}{m^{2}} \sum_{k=0}^{m} \sum_{a=-k}^{k} \exp (-\imath a x)=\sum_{n} \delta(x-2 \pi n)
$$

donde $\delta$ es la función delta de Dirac. Se puede comprobar que esta identidad se cumple para cualquier $x \neq 2 \pi n$ reagrupando los términos exponenciales. Para el caso $x=2 \pi n$ es evidente.

Utilizando la Ecuación 2.11, solamente sobreviven aquellos términos de la Ecuación 2.10 para los que se cumple $C_{a}=2 \pi n$ y $C_{b}=2 \pi k$ simultáneamente. Por esta razón, el problema de minimización de la energía de la monocapa se reduce a minimizar $\exp \left(-{ }_{2} \mathrm{Gr}_{0}\right)$ para un número finito de parejas $(R, \alpha)$. Una consecuencia de esto es el desacoplamiento de las variables $\left(x_{0}, y_{0}\right)$ de $(R, \alpha)$, lo que hace más sencillo el problema de minimización. Para encontrar las parejas $(R, \alpha)$ que sobreviven, se tiene que resolver un sistema de dos ecuaciones no lineales empezando por el primer término de Fourier con coeficiente $V_{G_{0}}\left(G_{0}=\frac{4 \pi}{\sqrt{3} l}\right)$, donde $l$ es la constante de red del sustrato. Por ejemplo, el primer componente del primer término 
de Fourier con longitud $G_{0}$ es $\cos \left(G_{0} y\right)$. Tomando la componente $y$ del vector real $\mathbf{r}$ desde la Ecuación 2.6 y resolviendo simultáneamente $C_{a}=2 \pi n$ y $C_{b}=2 \pi k$, donde $C_{a}$ y $C_{b}$ provienen de la Ecuación 2.8, el sistema de ecuaciones queda como:

$$
\left\{\begin{array}{l}
2 G_{0} R \sin \alpha=2 \pi n \\
G_{0} R(\sqrt{3} \cos \alpha+\sin \alpha)=2 \pi k
\end{array}\right.
$$

Este sistema tiene un número infinito de soluciones, pero en realidad estamos interesados en un intervalo restringido de $R$. Típicamente, en el caso de adsorción atómica y de moléculas pequeñas, $l \leq R \leq 2 \sqrt{3} l$. Este intervalo corresponde a un rango de cobertura de $\frac{1}{12} \leq \theta \leq 1$.

Aquí vamos a analizar numéricamente las soluciones para los términos de Fourier con las primeras seis capas de vectores recíprocos, empezando con vectores de longitud $G_{0}$. Cuando $R$ es restringido a un intervalo cerrado, $E_{\text {atom }}$ tiene un número finito de mínimos, debido a la identidad en la Ecuación 2.11. Encontramos que los términos de Fourier con los vectores recíprocos de la misma longitud dan las mismas soluciones. Esto significa que cada capa de vectores recíprocos da un número determinado de soluciones. La primera capa de vectores con longitud $G_{0}$ vuelve mínimas las estructuras simples conmensurables (con un átomo por celda unitaria). Para $l \leq R \leq 2 \sqrt{3} l$, existen seis parejas $(R, \alpha)$ : $(1,0),(\sqrt{3}, 30),(2,0),(\sqrt{7}, 19.11),(3,0),(2 \sqrt{3}, 30)$. El valor de $R$ se reporta en unidades de la constante de red del sustrato y $\alpha$ está en grados. En la notación tradicional de ciencia de superficies [1], estas estructuras se llaman $(1 \times 1),(\sqrt{3} \times \sqrt{3}) R 30^{\circ},(2 \times 2),(\sqrt{7} \times$ $\sqrt{7}) R 19.11^{\circ},(3 \times 3)$ y $(2 \sqrt{3} \times 2 \sqrt{3}) R 30^{\circ}$, respectivamente. La posición mínima de $\left(x_{0}, y_{0}\right)$ de la monocapa depende del signo del coeficiente $V_{G_{0}}$ : para $V_{G_{0}}>0$, el sitio mínimo está entre tres átomos del sustrato, mientras que para $V_{G_{0}}<0$, el mínimo está localizado directamente sobre un átomo del sustrato (vea la Figura 1.3, donde el potencial átomo-superficie se muestra para el caso $V_{G_{0}}>0$ ).

Los términos de Fourier de orden superior hacen mínimas las estructuras conmensurables 
de orden superior (con más de un átomo por celda unitaria). Un hecho interesante es que las estructuras conmensurable simples, descritas arriba, son mínimos de energía para todos los términos de Fourier. De esta forma, las estructuras conmensurables con un átomo por celda unitaria siempre serán mínimos globales, independientemente de los valores de los coeficientes $V_{G}$. La segunda capa de vectores recíprocos estabiliza las monocapas con tres átomos por celda unitaria. Por otro lado, la tercera capa estabiliza las estructuras con cuatro átomos por celda. Las estructuras con tres átomos por celda vuelven a ser mínimas para la quinta capa, mientras que las estructuras con cuatro átomos se vuelven mínimas para la sexta capa. Los modelos de todas las estructuras mínimas para cualquier capa de vectores recíprocos consideradas en este trabajo se muestran en la Figura 2.1. La Figura 2.2 muestra las mismas estructuras de la Figura 2.1 en forma topográfica (vea el pie de figura para mayor información). La Tabla 2.1 muestra las parejas $(R, \alpha)$ para todas las estructuras obtenidas para cualquier término de Fourier (hasta seis capas de vectores).

La gráfica de todas las parejas estables $(R, \alpha)$ se muestra en la Figura 2.3. El tamaño de los círculos solamente sirve como una guía para el ojo e indica la energía relativa de las estructuras considerando un decaimiento simple en los coeficientes de Fourier (círculos más grandes corresponden a energías más bajas). Los círculos no están dibujados a escala y solamente pueden asociarse a un comportamiento cualitativo. Las estructuras conmensurables simples son de mínima energía para las seis capas de vectores recíprocos, por lo que están marcadas con los círculos más grandes. El valor de coeficientes $V_{G}$ en un sistema real va a cambiar el tamaño relativo de los círculos. Aún así es importante notar que los $(R, \alpha)$ mínimos no dependen de los valores particulares de los $V_{G}$, los coeficientes solamente cambian la estabilidad relativa de distintas estructuras. La Figura 2.3 presenta un comportamiento interesante, ya que se puede observar una clara simetría hexagonal si se conectan los puntos 

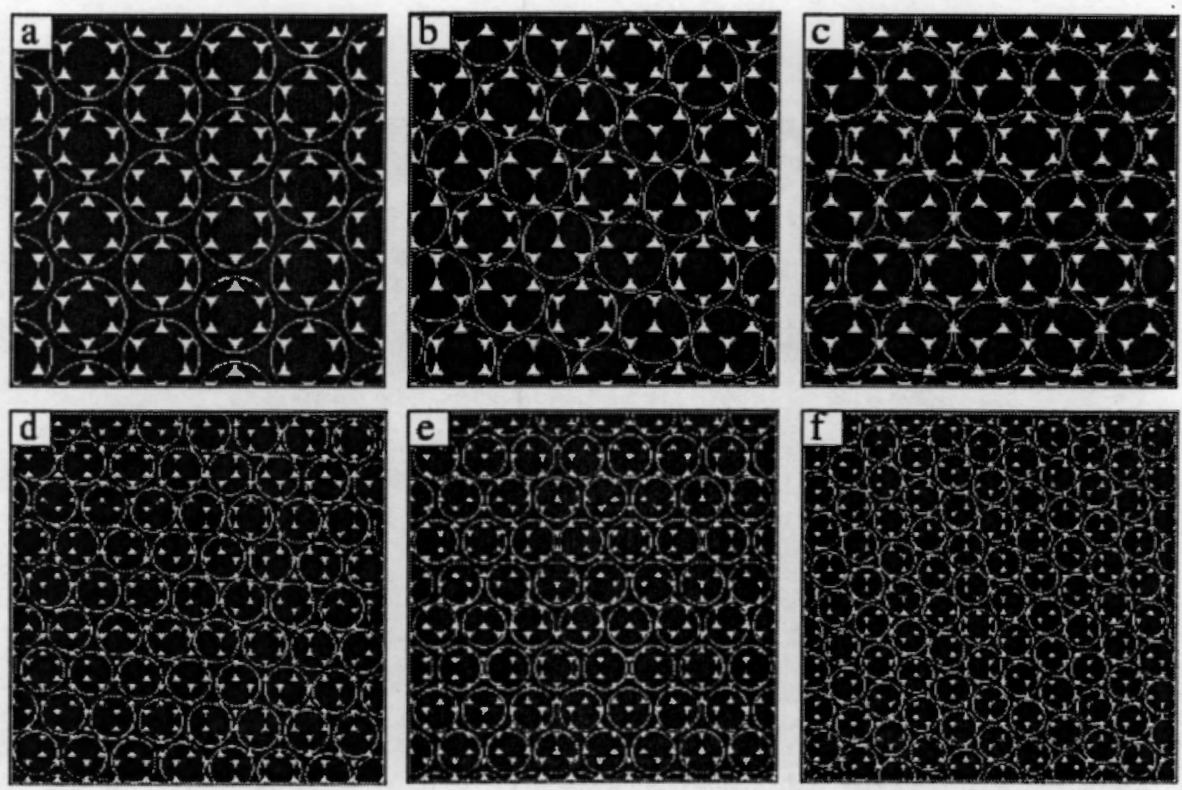

Figura 2.1: Modelos de estructuras de mínima energía para términos de Fourier con las primeras seis capas de vectores recíprocos. Los círculos sólidos negros son los átomos del sustrato, mientras que los círculos blancos más grandes son los átomos del adsorbato. Se muestra el caso de $\left(x_{0}, y_{0}\right)=$ $(0,0)$, el cual se obtiene al poner un átomo de adsorbato directamente sobre un átomo del sustrato. El número de estructuras distintas con la misma simetría (mismo registro atómico pero distinta distancia interatómica) para $l \leq R \leq 2 \sqrt{3} l$ está indicado entre paréntesis y. están especificadas explícitamente en la Tabla 2.1 a) Estable para las seis capas (6). b) Estable para la 2da y 5 ta capa (8). c) Estable para 3ra y 6ta capa (11). d) Estable para la 4ta capa (42). e) Estable para la 5ta capa (22). f) Estable para la 6ta capa (22). 

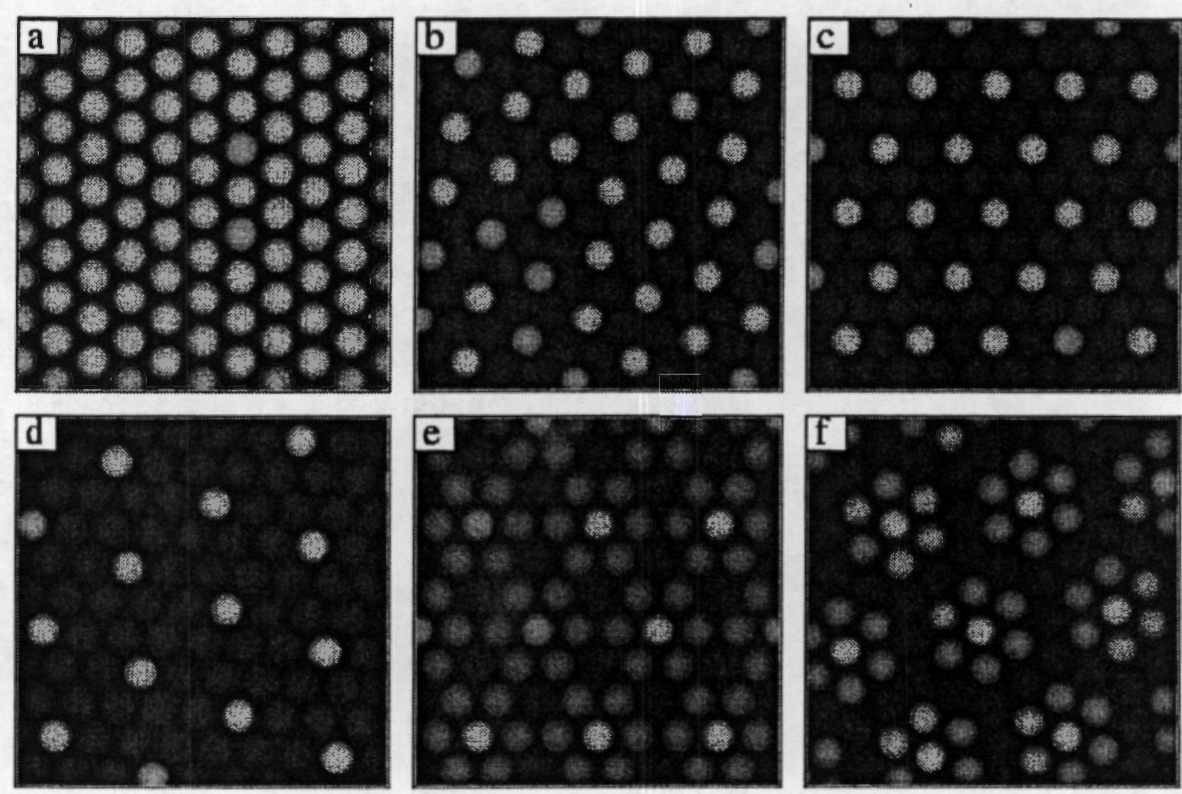

Figura 2.2: Las mismas estructuras de la Figura 2.1 mostradas en forma topográfica. La escala de colores varía de blanco (sitio a) a negro (sitio f). Nótese la disminución del grado de simetría con los términos de Fourier de orden superior. Las imágenes fueron generadas con el programa ALSA. Ver la Ref. [16] para mas detalles. 
Tabla 2.1: Los valores de parejas $(R, \alpha)$ de estructuras de mínima energía para distintas combinaciones de términos de Fourier, hasta la sexta capa de vectores recíprocos para $l \leq R \leq 2 \sqrt{3}$, donde $l$ es la constante de red de la superficie. $R$ se reporta en unidades de $l$, mientras que $\alpha$ se reporta en grados. La primera hilera muestra las estructuras conmensurables simples con un átomo por celda unitaria, los cuales son estables para cualquier capa de vectores recíprocos. La segunda hilera muestra las estructuras con tres átomos por celda unitaria. Las siguientes hileras muestran las estructuras de mínima energía para las capas de vectores subsecuentes.

\begin{tabular}{|c|c|c|c|c|c|}
\hline Seis Capas & $\begin{array}{l}(1,0.00) \\
(2 \sqrt{3}, 30.00)\end{array}$ & $(\sqrt{9,30.00)}$ & $(2,0.00)$ & $(\sqrt{7,19.11})$ & $(3,0.00)$ \\
\hline $2 \mathrm{da}+5 \mathrm{ta}$ & $\begin{array}{l}(2 / \sqrt{3}, 30.00) \\
(2.8868,30.00)\end{array}$ & $\begin{array}{l}(1.5275,10.89) \\
(3.0551,10.89)\end{array}$ & $\begin{array}{l}(2.0817,16.10) \\
(3.2146,21.05)\end{array}$ & $(2.3094,30.00)$ & $(2.5166,6.59)$ \\
\hline $3 \mathrm{ra}+6 \mathrm{ta}$ & $\begin{array}{l}(1.3229,19.11) \\
(2.5000,0.00) \\
(3.2787,7.59)\end{array}$ & $\begin{array}{l}(1.5000,0.00) \\
(2.5981,30.00)\end{array}$ & $\begin{array}{l}(1.8028,13.90) \\
(2.7839,8.95)\end{array}$ & $\begin{array}{l}(2.1794,23.41) \\
(3.0414,25.28)\end{array}$ & $\begin{array}{l}(2.2913,10.89) \\
(3.1225,16.10)\end{array}$ \\
\hline 4 ta & $\begin{array}{l}(1.0000,21.79) \\
(1.5119,19.11) \\
(1.9640,10.89) \\
(2.2991,15.61) \\
(2.4785,26.70) \\
(2.8536,12.52) \\
(3.0237,19.11) \\
(3.2733,10.89) \\
(3.4017,19.11)\end{array}$ & $\begin{array}{l}(1.1339,19.11) \\
(1.6475,17.48) \\
(2.0000,21.79) \\
(2.2991,6.18) \\
(2.6186,10.89) \\
(2.8536,25.69) \\
(3.0938,28.68) \\
(3.2950,17.48) \\
(3.4641,8.21)\end{array}$ & $\begin{array}{l}(1.3093,10.89) \\
(1.6475,4.31) \\
(2.1044,10.16) \\
(2.3604,24.79) \\
(2.6458,2.68) \\
(2.9520,14.56) \\
(3.0938,6.89) \\
(3.2950,4.31)\end{array}$ & $\begin{array}{l}(1.3628,27.00) \\
(1.7321,8.21) \\
(2.1044,28.05) \\
(2.3604,3.00) \\
(2.7255,27.00) \\
(2.9520,7.22) \\
(3.2293,13.29) \\
(3.3594,2.11)\end{array}$ & $\begin{array}{l}(1.3628,5.21) \\
(1.8898,19.11) \\
(2.2678,19.11) \\
(2.4785,11.52) \\
(2.7255,5.21) \\
(3.0000,21.79) \\
(3.2293,24.92) \\
(3.3594,23.90)\end{array}$ \\
\hline $5 \mathrm{ta}$ & $\begin{array}{l}(1.2019,13.90) \\
(1.8559,8.95) \\
(2.4037,13.90) \\
(2.9059,23.41) \\
(3.3333,0.00)\end{array}$ & $\begin{array}{l}(1.3333,0.00) \\
(2.0276,25.28) \\
(2.6034,26.33) \\
(2.9627,17.00) \\
(3.3830,9.83)\end{array}$ & $\begin{array}{l}(1.4530,23.41) \\
(2.1858,7.59) \\
(2.6667,0.00) \\
(3.1798,27.00)\end{array}$ & $\begin{array}{l}(1.6667,0.00) \\
(2.3333,0.00) \\
(2.7285,12.22) \\
(3.1798,5.21)\end{array}$ & $\begin{array}{l}(1.7638,19.11) \\
(2.3333,21.79) \\
(2.8480,5.82) \\
(3.2830,15.30)\end{array}$ \\
\hline 6 ta & $\begin{array}{l}(1.0408,16.10) \\
(1.8930,22.41) \\
(2.4664,24.18) \\
(2.9297,20.17) \\
(3.3292,25.69)\end{array}$ & $\begin{array}{l}(1.2583,6.59) \\
(2.0207,30.00) \\
(2.5658,13.00) \\
(3.0139,5.50) \\
(3.4034,17.27)\end{array}$ & $\begin{array}{l}(1.4434,30.00) \\
(2.0207,8.21) \\
(2.7538,24.79) \\
(3.1754,30.00)\end{array}$ & $\begin{array}{l}(1.6073,21.05) \\
(2.2546,3.67) \\
(2.7538,3.00) \\
(3.2532,2.54)\end{array}$ & $\begin{array}{l}(1.7559,4.72) \\
(2.3629,17.78) \\
(2.8431,14.70) \\
(3.3292,12.52)\end{array}$ \\
\hline
\end{tabular}




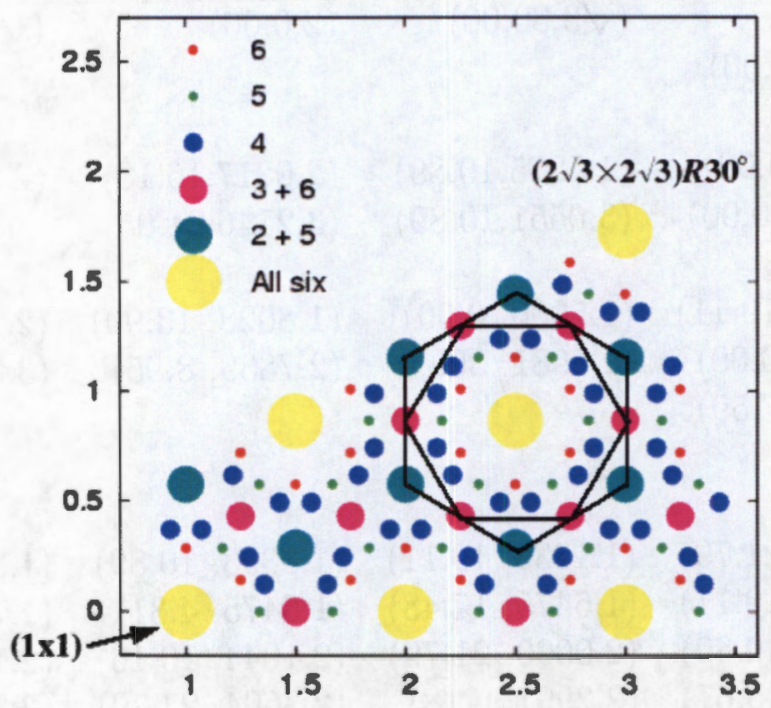

Figura 2.3: Gráfica de parejas estables $(R, \alpha)$ en coordenadas polares. Los valores de las variables están restringidos por $l \leq R \leq 2 \sqrt{3} l$ y $0^{\circ} \leq \alpha \leq 30^{\circ}$. Este límite puede ser impuesto para $\alpha$ debido a la simetría hexagonal de la monocapa. La leyenda de la figura especifica las capas de vectores recíprocos para los cuales una cierta estructura es de mínima energía. Se puede ver claramente patrones hexagonales si se conectan los puntos correspondientes a las estructuras con la misma simetría. El tamaño de círculos es solamente una guía para el ojo, considerando que los coeficientes de Fourier muestran un comportamiento decreciente. El tamaño en este caso cambia de 6 a 1 . 


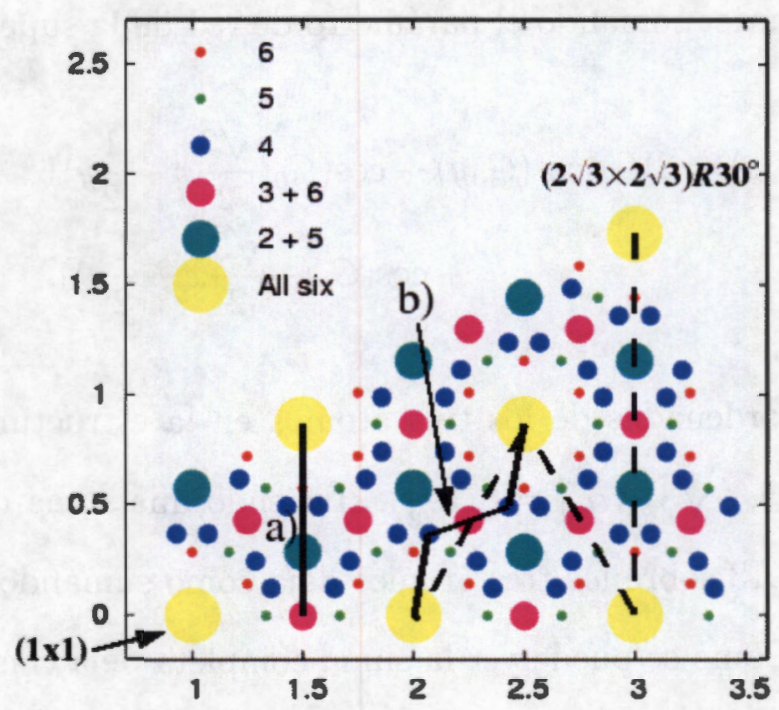

Figura 2.4: La misma información de la Figura 2.3 con distintas trayectorias propuestas para los sistemas I-Pt(111) y $\mathrm{K}, \mathrm{Rb}$ y $\mathrm{Cs}$ en $\mathrm{Ag}(111)$. Una posible trayectoria para I-Pt(111) está marcada por a). La línea partida indica la trayectoria más simple para la rotación de metales alcalinos en superficie $\mathrm{Ag}(111)$. Por otro lado, siguiendo la trayectoria marcada por b) se puede obtener un buen grado de concordancia con los datos experimentales de Leatherman et al. [19]. 
correspondientes a las estructuras de misma simetría (correspondientes a las mismas capas de vectores recíprocos).

Así mismo, es interesante observar que los términos de Fourier de orden bajo se cancelan para estructuras conmensurables desde cierto número de átomos por celda unitaria. Por ejemplo, el primer término de Fourier se cancela para todas las estructuras hexagonales rígidas con más de un átomo por celda unitaria primitiva. Para analizar esto en más detalle vamos a tomar el caso de la estructura $(\sqrt{7} \times \sqrt{7}) R 19.11^{\circ}$ con tres átomos por celda unitaria. El primer término de Fourier tomando el parámetro de red de la superficie $l$ igual a 2 es:

$$
\begin{array}{r}
V(x, y)=2 V_{G_{0}}\left[\cos \left(G_{0} y\right)+\cos \left(G_{0}\left(\frac{\sqrt{3}}{2} x-\frac{1}{2} y\right)\right)\right. \\
\left.+\cos \left(G_{0}\left(-\frac{\sqrt{3}}{2} x-\frac{1}{2} y\right)\right)\right]
\end{array}
$$

donde $G_{0}=\frac{2 \pi}{\sqrt{3}}$. Las coordenadas de los tres átomos en la estructura $(\sqrt{7} \times \sqrt{7}) R 19.11^{\circ}$ son: $a_{1}=\left(3, \frac{1}{\sqrt{3}}\right), a_{2}=(5,-\sqrt{3})$ y $a_{3}=\left(6, \frac{2}{\sqrt{3}}\right)$. Haciendo una suma del primer término de Fourier de la Ecuación 2.13 sobre los tres átomos deja como sumandos: -0.5, 1.0, -0.5, -0.5, $1.0,-0.5,-0.5,1.0,-0.5$. Como se puede ver la suma completa deja como resultado cero.

Similar a la situación anterior, los términos correspondientes a la segunda y tercera capa de vectores recíprocos se cancelan para estructuras con más de tres átomos por celda. Esta conclusión puede ser ampliada al observar las estructuras en las Figura 2.1 y 2.2 y su relación con los términos específicos de Fourier.

Para comparar esta teoría con sistemas reales, vamos a utilizar datos experimentales de monocapas de yodo en $\mathrm{Pt}(111)$ y $\mathrm{K}, \mathrm{Rb}, \mathrm{Cs}$ en la superficie $\mathrm{Ag}(111)$. El sistema I$\mathrm{Pt}$ (111) forma cuatro estructuras conmensurables distintas: $(\sqrt{3} \times \sqrt{3}) R 30^{\circ}$ con un átomo por celda, $(\sqrt{7} \times \sqrt{7}) R 19.11^{\circ}$ con tres átomos por celda, $(3 \times 3)$-sym y $(3 \times 3)$-asym con cuatro átomos por celda $[37,59,60]$. Los metales alcalinos forman una gran variedad de estructuras conmensurables e inconmensurables en $\mathrm{Ag}(111)$ [19,61]. Ambos sistemas poseen 
distintas estructuras de alto orden de conmensurabilidad, lo que indica la complejidad de la expansión de Fourier del potencial átomo-superficie. Se cree que las fuerzas dominantes en la interacción lateral en la monocapa son repulsivas. Para los metales alcalinos, los efectos de transferencia de carga más las interacciones mediadas por el sustrato son responsables de esta interacción repulsiva efectiva $[19,61,62]$, mientras que distintas fuerzas contribuyen en el caso de la interacción entre los halógenos $[37,47,63,64]$. Como ya mencionamos anteriormente, las fuerzas repulsivas favorecen a la formación de monocapas hexagonales. Por lo mismo, la teoría desarrollada en esta sección parecería ser más adecuada para este tipo de sistemas.

La Figura 2.4 muestra los mismos datos de la Figura 2.3 junto con algunas trayectorias probables ${ }^{\mathrm{a}}$ para I-Pt(111) y $\mathrm{K}, \mathrm{Rb}$ y $\mathrm{Cs}$ en $\mathrm{Ag}(111)$. Es claro que las trayectorias observadas experimentalmente dependen de valores exactos de los coeficientes $V_{G}$ y las interacciones laterales en la monocapa. Es por esto que las comparaciones con los experimentos presentadas aquí son mayormente cualitativas. Aún así, se pueden obtener conclusiones interesantes como resultado de la comparación de la teoría analítica con sistemas experimentales reales. Se puede observar que en el caso de I-Pt(111), una trayectoria obvia es una línea recta en coordenadas polares en el espacio $(R, \alpha)$. Para $\mathrm{K}, \mathrm{Rb}$ y $\mathrm{Cs}$ en $\mathrm{Ag}(111)$, diferentes trayectorias son posibles entre las estructuras $(2 \times 2)$ y $(\sqrt{7} \times \sqrt{7}) R 19.11^{\circ}$, pasando por distintas estructuras conmensurables. Se obtiene un acuerdo excelente con los datos experimentales de Leatherman et al. [19] siguiendo la trayectoria marcada por b) en la Figura 2.4, la cual, transformada al espacio cartesiano, es muy similar a la curva experimental de $\alpha$ contra $R$ [19].

Sin embargo, para demostrar que las interacciones sustrato-adsorbato juegan un papel importante en la determinación del comportamiento rotacional de los metales alcalinos en $\mathrm{Ag}(111)$, se necesitan conocer los valores exactos de los coeficientes $V_{G}$ para las estructuras $(2 \times 2)$ y $(\sqrt{7} \times \sqrt{7}) R 19.11^{\circ}$. Esto puede ser posible mediante el empleo de TFD, la cual

${ }^{a}$ La palabra trayectoria se usa para indicar la función del ángulo de rotación contra la distancia interatómica, la cual conecta dos estructuras y puede pasar por otras estructuras de mínima energía en su camino. 
se ha utilizado con frecuencia para la adsorción de halógenos $[43,47,63,65-67]$ y metales alcalinos $[62,68,69]$ en superficies metálicas.

Doering [31] propuso un modelo basado en la simetría de la monocapa e identificó una trayectoria especial en el espacio $(R, \alpha)$ para una monocapa hexagonal en una superficie (111). Grey y Bohr $[32,33]$ usaron un modelo de un solo componente de Fourier y, considerando el tamaño finito de las terrazas de superficie, encontraron una trayectoria similar a la propuesta por Doering. La teoría propuesta en esta sección extiende los modelos de Doering y Grey-Bohr mostrando la existencia de muchas trayectorias distintas en el espacio $(R, \alpha)$ cuando se usa una expansión de Fourier con términos de orden superior.

\subsection{Relación de la expansión de Fourier con los "núme- ros mágicos"}

En la sección anterior, se obtuvieron parejas de $(R, \alpha)$ de mínima energía correspondientes a cada término de Fourier. Asímismo, se demostró que cada término de una capa de vectores recíprocos puede asociarse a una estructura conmensurable con cierto número de átomos por celda unitaria, aunque esta asociación se hizo de una forma un tanto empírica. En esta sección profundizaremos en la asociación de cada término de la expansión de Fourier a estructuras conmensurables con los "números mágicos" de la secuencia de números hexagonales que fueron introducidos en la sección 2.1.

Para facilitar la presentación, vamos a llamar $\mathcal{F}_{\boldsymbol{i}}$ al término de Fourier asociado con una capa de vectores recíprocos de longitud $|G|=D G_{0}$. De esta forma, el potencial átomosuperficie se puede escribir como,

$$
V(\mathbf{r})=\sum_{i} V_{G_{i}} \mathcal{F}_{i}(\mathbf{r})
$$


donde el índice $i$ indica la posición de $D^{2}$ en el conjunto $\mathcal{H}$ (vea la sección 2.1). También se supone que los coeficientes de Fourier $V_{G}$ del potencial átomo-superficie se conocen hasta una longitud particular $|G| \leq D_{\text {lim }} G_{0}$ para nuestro sistema de interés.

La asociación de los términos de Fourier al número de átomos por celda unitaria es obvia en el caso de un átomo de adsorbato $\left(N_{a d s}=1\right)$ posicionado en una celda de sustrato con $N_{\text {sus }}$ átomos. La energía en ese caso es una suma sobre toda la serie de Fourier, donde todos los términos de la serie contribuyen a la energía del sistema. Sería interesante poder generalizar este argumento a celdas con $N_{\text {ads }}>1$. Utilizamos el argumento para obtener el sistema de ecuaciones (Ecuación 2.12) en la sección anterior y sustituimos la expresión general para un vector recíproco G. De esta forma, los valores mínimos $(R, \alpha)$ para los términos de Fourier asociados a vectores recíprocos $G=\left(G_{x}, G_{y}\right)=G_{0}(0.5 \sqrt{3} b, a+0.5 b)$, con $|G|=\sqrt{G_{x}^{2}+G_{y}^{2}}=D G_{0}$ (una capa de vectores) son dados por las soluciones al siguiente sistema de ecuaciones ${ }^{\mathbf{a}}$ :

$$
\left\{\begin{array}{l}
G_{x} \cos \alpha+G_{y} \sin \alpha=\frac{2 \pi n}{R} \\
G_{y} \cos \alpha-G_{x} \sin \alpha=\frac{2 \pi(2 k-n)}{\sqrt{3} R}
\end{array}\right.
$$

Después del desarrollo algebráico, la solución a este sistema de ecuaciones se puede escribir como:

$$
\begin{aligned}
& R=\sqrt{\frac{n^{2}+k^{2}+n k}{a^{2}+b^{2}+a b}} \\
& \alpha=\arctan \left[\frac{-\sqrt{3} n G_{y}-(2 k+n) G_{x}}{-\sqrt{3} n G_{x}+(2 k+n) G_{y}}\right]
\end{aligned}
$$

Se puede notar que $R$ es una expresión de tipo $R=\sqrt{\frac{N_{\text {mas }}}{N_{a d e}}}$, lo que nos lleva al siguiente Lema.

Lema. El término de Fourier de longitud $D G_{0}$ contribuye a la energía de una monocapa

a Para formar este sistema de ecuaciones, se resuelve simultaneamente $C_{a}=2 \pi n$ y $C_{b}=2 \pi k$, donde $C_{a}$ y $C_{b}$ provienen de la Ecuación 2.8. 
conmensurable con $N_{\text {ads }}=D^{2}$ partículas por celda unitaria, con un ángulo de rotación $\alpha$ dado por la ecuación anterior con respecto al sustrato.

En este punto se puede generar una secuencia de términos de Fourier que corresponden a una monocapa conmensurable con $D^{2}$ partículas. Primero, se necesita ver que la identidad $\frac{N_{a d d, 1}}{N_{s u b, 1}}=\frac{N_{a d s, 2}}{N_{s u b, 2}}$ implica que $N_{a d s, 2}=m N_{a d s, 1}$ y $N_{s u b, 2}=m N_{s u b, 1}$, donde $m \in \mathcal{H} . N_{a d s, 2}$ y $N_{s u b, 2}$ pertenecen a $\mathcal{H}$, debido a la propiedad de cerradura (vea la sección 2.1). Por lo tanto, para cualquier sistema monocapa-superficie dado, todas las estructuras expandidas (no primitivas) con el mismo grado de cobertura que una estructura primitiva se pueden obtener multiplicando por un $m \in \mathcal{H}$ progresivamente mayor. Vamos a considerar el caso de una monocapa con $N_{\text {ads }}=1$ sobre el sustrato con $N_{\text {sus }}$ partículas una vez mas. Utilizando el lema anterior, los términos de Fourier de la primera capa de vectores recíprocos contribuyen en este caso. Expandemos la celda unitaria multiplicando $N_{\text {ads }}$ y $N_{\text {sus }}$ por un $m \in \mathcal{H}$. En este caso, el término de Fourier asociado con $m N_{a d s}$ también contribuye. Este procedimiento se puede repetir para cualquier $m \in \mathcal{H}$. Por lo mismo, es claro que todos los términos de Fourier contribuyen en el caso de $N_{a d s}=1$. Ahora vamos a tomar una monocapa con $N_{a d s}=3$ y $N_{\text {sus }}$ arbitrario. En este caso, los términos de Fourier asociados a la segunda capa de vectores recíprocos contribuyen. Repetimos el procedimiento de multiplicar por $m$ de nuevo. Ahora se puede ver que los términos de Fourier asociados a las capas de vectores $2,5,6,10, \ldots$ contribuyen. Utilizando un razonamiento idéntico para el caso $N_{a d s}=4$, los términos de las capas $3,6,8,13, \ldots$ contribuyen. Este tipo de construcción permite generar un algoritmo para encontrar todos los términos de Fourier que contribuyen a la energía de una monocapa hexagonal rígida con un número dado de partículas. Llamaremos $\mathcal{H}_{N}$ el conjunto formado por todos los términos de Fourier $\mathcal{F}_{i}$ los cuales se conservan para una monocapa con $N_{a d s}=N$. Es evidente que $\mathcal{H}_{N} \subseteq \mathcal{H}$.

Hasta el momento, no se ha mencionado nada sobre el ángulo de rotación de la monocapa. Las monocapas expandidas se generan con el mismo ángulo que las primitivas. Sin embargo, 
dada una monocapa con $N_{a d s}$ partículas, el ángulo de rotación $\alpha$ no es único. La mejor forma de ilustrarlo es por medio de un ejemplo. Tomemos el sistema con $N_{\text {ads }}=21$ y $N_{\text {sus }}=49$. Dependiendo de los valores de $a$ y $b$ utilizados para generar $N_{a d s}$, el ángulo de rotación puede tomar dos valores diferentes según la Ecuación 2.16: $10.893^{\circ}$ y $27.320^{\circ}$. El primero corresponde a una estructura reducida con $N_{\text {ads }}=3$ y $N_{\text {sus }}=7$ con la energía dada por $\mathcal{H}_{3}$, mientras que la segunda es dada por $N_{\text {ads }}=21$ y $N_{\text {sus }}=49$ con la energía igual a $\mathcal{H}_{10}$.

Finalmente, los mínimos traslacionales $\left(x_{0}, y_{0}\right)$ para las monocapas hexagonales con $N$ átomos se pueden obtener como soluciones a,

$$
\min \sum_{\mathcal{F}_{i} \in \mathcal{H}_{N}} V_{G_{i}} \exp \left[-\imath\left(G_{i, x} x_{0}+G_{i, y} y_{0}\right)\right]
$$

Nótese que uno necesita los valores exactos de los coeficientes de Fourier $V_{G}$ hasta este punto unicamente para resolver la ecuación anterior y obtener los valores mínimos de $\left(x_{0}, y_{0}\right)$. La teoría antes de la Ecuación 2.17 es exacta para valores arbitrarios de los coeficientes $V_{G}$. Claramente, las magnitudes relativas de los $V_{G}$ son importantes para especular sobre la posibilidad de existencia de una monocapa conmensurable de una cierta simetría. 


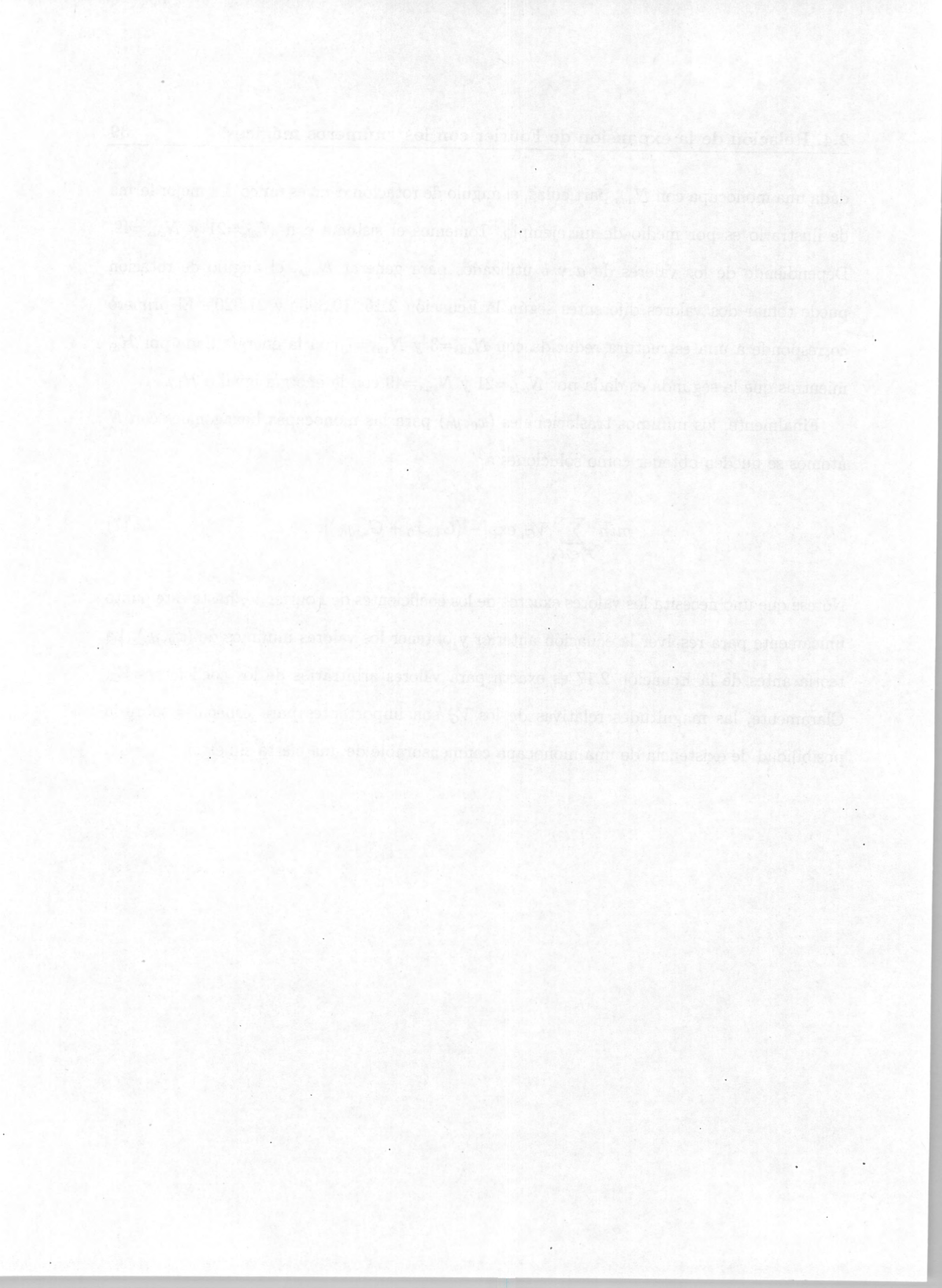




\section{Capítulo 3}

\section{Simulaciones de monocapas de}

\section{halógenos con TFD}

En este capítulo se estudia la adsorción de yodo en la superficie Pt(111) por medio de cálculos basados en la Teoría de Funcionales de la Densidad (TFD). En la primera sección se da una breve introducción a la metodología TFD empleada. En la segunda sección se muestran los resultados de la adsorción de un átomo de yodo en las celdas $(2 \times 2)$ y $(3 \times 3)$ de la superficie $\mathrm{Pt}(111)$. Se hace un análisis de energías de adsorción y transferencia de carga para cuatro sitios distintos, mostrados en la Figura 1.1. Esto permite investigar el origen de la interacción átomo-superficie en el sistema I-Pt(111). En la tercera sección se hacen simulaciones de estructuras ordenadas del sistema I-Pt(111) en celdas que se han observado experimentalmente.

\subsection{Metodología TFD}

En este trabajo se usa un método que utiliza condiciones periódicas en el contexto de la TFD para estudiar la adsorción de yodo en la superficie Pt(111). En este método, la función 
de onda es una expansión en ondas planas y tiene la simetría correcta de la celda unitaria empleada.

Hay que mencionar que hoy en día existen distintas formas de estudiar un problema de adsorción de átomos en superficies. Se pueden enumerar las dos más importantes: el modelo de cúmulos finitos y el modelo de losa con condiciones periódicas en dos dimensiones. La diferencia entre los dos métodos radica en la representación de la superficie que se utiliza. En el primero, la superficie se describe por medio de un cúmulo de átomos, el cual es generalmente pequeño y en el segundo, la superficie se describe como una losa de superficie con un número finito de capas, pero cada una de estas capas es infinita en las dimensiones $x$ y $y$. Para ambos métodos, existen distintas formas de representar la función de onda. Las formas más usadas son las funciones localizadas (generalmente gaussianas) y las ondas planas. Las funciones gaussianas son los orbitales naturales para describir sistemas localizados y su uso proviene de la química cuántica. Por otro lado, el empleo de ondas planas proviene de la física del estado sólido y son las funciones naturales para describir funciones con una periodicidad dada. Vale la pena mencionar que ambos tipos de base (funciones localizadas y ondas planas) pueden ser utilizados para el modelo periódico, aunque la elección de ondas planas es más natural [70]. En cuanto a la validez de las dos formas de representar una superficie, es claro que el modelo de cúmulos finitos es una aproximación más alejada de la realidad cuando se trata de describir la estructura electrónica de superficies extendidas. Por ejemplo, dependiendo de la naturaleza de la superficie (el carácter metálico) el tamaño del cúmulo necesario para una representación fiel de la superficie puede ser muy grande. Por otro lado, las funciones localizadas no son lo suficientemente buenas para representar el origen deslocalizado de los electrones para un material metálico y las ondas planas ofrecen una base más adecuada para representar una función de onda con una periodicidad dada.

En el caso del modelo periódico, al tener un sistema infinito, se tendrá también un número infinito de electrones. Para poder resolver este problema se utiliza el teorema de Bloch [71], 
y se hace un mapeo del sistema en espacio real al espacio recíproco (espacio de Fourier), por lo cual el problema original de un número infinito de electrones se reduce a un problema de $N$ electrones en una celda unitaria. La función de onda se representa por una suma de ondas planas:

$$
\psi_{i, \mathbf{k}}(\mathbf{r})=\sum_{\mathbf{G}} c_{i, \mathbf{k}+\mathbf{G}} \exp [i(\mathbf{k}+\mathbf{G}) \mathbf{r}]
$$

donde $\mathbf{G}$ son los vectores de la celda recíproca definidos por la ecuación $\mathbf{G} \cdot 1=2 \pi m$, para todo 1 , donde $l$ es un vector de la celda en espacio real, $m$ es un número entero y $\mathbf{k}$ es cualquier vector dentro de la primera zona de Brillouin. En principio, el conjunto de base para un $\mathbf{k}$ dado es infinito, pero en la práctica el conjunto de ondas planas se restringe a una esfera en espacio recíproco definida por un parámetro conocido como energía de truncamiento o energía de corte, $q_{c}$. Para todos los valores de $\mathbf{G}$ usados en la expansión de la Ecuación 3.1 se cumple que

$$
\frac{1}{2}|\mathbf{k}+\mathbf{G}|^{2} \leq q_{c}
$$

De esta manera, la convergencia de un cálculo con respecto al conjunto de ondas planas se puede controlar variando únicamente el valor de $q_{c}$. Esta es una ventaja muy importante respecto a muchos otros métodos en los cuales las propiedades calculadas muestran frecuentemente una alta sensibilidad a pequeños cambios en el conjunto de base.

Al utilizar el teorema de Bloch, en vez de resolver un problema de número infinito de electrones, se resuelve un problema de un número finito $N$ de electrones en un número infinito de puntos $k$ en el espacio recíproco (primera zona de Brillouin). Generalmente, se hace un mapeo en un número finito de puntos especiales en esta zona (puntos $k$ ) empleando el esquema de Monkhorst-Pack [72]. En el caso de la adsorción sobre una superficie, estos puntos se escogen sobre el plano, ya que en el modelo de sistema extendido para una superficie la tercera dimensión se escoge de tal forma que se minimice la interacción entre las imágenes periódicas de ésta. 
Como la función de onda es una expansión en ondas planas, se requiere un número elevado de éstas para representar los cambios de la densidad electrónica cerca del núcleo, por lo que se emplean pseudopotenciales y solamente se tratan explícitamente unos cuantos electrones de valencia para cada átomo del sistema. Esta aproximación será valida para el caso donde solamente algunos electrones de valencia participan en los enlaces químicos. Por lo que el efecto del núcleo y los electrones internos de un átomo, que ejercen un potencial iónico fuerte sobre los electrones de valencia, se puede sustituir por un pseudopotencial más débil, que actúe sobre un conjunto de pseudofunciones de onda de valencia en lugar de las funciones de onda de valencia verdaderas. Las pseudofunciones correspondientes a este potencial modificado no tienen las oscilaciones de las funciones de onda verdaderas, por lo que se reduce de manera importante el número de ondas planas requeridas para su representación. Los pseudopotenciales atómicos utilizados en este trabajo se conocen como pseudopotenciales con conservación de la norma $[73,74]$, lo que implica las siguientes propiedades:

(1) La parte radial de la pseudofunción (que sustituye a la función de onda verdadera) de onda $R_{l}^{p s}(r)$ con momento angular $l$, es igual a la parte radial de la función de onda atómica $R_{l}(r)$, a partir de un radio de core $\left(r_{c}\right)$ medido desde el núcleo atómico; esto es, $R_{l}^{p s}(r)=R_{l}(r)$ para $r \geq r_{c}$.

(2) La densidad de carga de la función atómica y de la pseudofunción deben ser iguales dentro del $r_{c}$; esto es, $\int_{0}^{r_{c}}\left|R_{l}^{p s}(r)\right|^{2} r^{2} d r=\int_{0}^{r_{c}}\left|R_{l}(r)\right|^{2} r^{2} d r$. Esta propiedad se conoce como conservación de la norma.

(3) Deben ser iguales los eigenvalores obtenidos con la pseudofunción $R_{l}^{p s}(r)$ y con la función de onda $R_{l}(r)$; esto es $\epsilon_{l}^{p s}=\epsilon_{l}$.

(4) Las derivadas logarítmicas de la función de onda verdadera y de la pseudofunción deben ser iguales después de $r_{c}$.

Hay varios esquemas para generar pseudopotenciales de este tipo, como el método de Troullier y Martins [74] y el método de Rappe y colaboradores (RRKJ) [75, 76]. Estos pro- 
cedimientos generan pseudopotenciales transferibles entre una amplia variedad de ambientes químicos, lo que caracteriza a los pseudopotenciales con conservación de la norma. La transferabilidad de estos pseudopotentiales se ha comprobado empiricamente por medio de cálculos numéricos.

Es importante mencionar que la aproximación de pseudopotenciales tiene la ventaja de que la remoción de los electrones internos implica el cálculo de un menor número de funciones de onda electrónicas. Sin embargo, esta aproximación también tiene la desventaja de no describir apropiadamente las propiedades que dependen de los electrones internos, como las propiedades ópticas y magnéticas.

\subsection{Un átomo en las celdas $(2 \times 2)$ y $(3 \times 3)$}

La adsorción de yodo en la superficie $\mathrm{Pt}(111)$ ha sido estudiada extensamente por distintas técnicas experimentales $[37,59,77-82]$, pero existen muy pocos estudios teóricos de primeros principios sobre este sistema $[83,84] .^{a} \mathrm{El}$ interés en este sistema se puede explicar por el hecho de que los procedimientos experimentales para su preparación y caracterización están bien establecidos, pero a la vez existen cuatro estructuras ordenadas diferentes con grados de cobertura distintos: $(\sqrt{3} \times \sqrt{3}) R 30^{\circ},(\sqrt{7} \times \sqrt{7}) R 19.11^{\circ},(3 \times 3)$-sym y $(3 \times 3)$-asym. Cada una de estas estructuras se caracteriza por tener uno o más sitios de adsorción $[77,79,81]$. Los resultados obtenidos para el sistema I-Pt(111) son muy similares en vacío, solución y aire [37]. Aunque los resultados experimentales sobre I-Pt(111) están bien establecidos, todavía faltan muchos detalles sobre la química de la adsorción y los arreglos ordenados. Por ejemplo, algunas de las preguntas sin resolver son: la naturaleza del enlace yodo-platino, la relajación de la superficie de platino, y la estabilidad de las distintas estructuras ordenadas. En esta sección se tratará de responder a algunas de estas interrogantes. La mayoría de estudios

\footnotetext{
"Esta sección está basada en el artículo Charge transfer and adsorption energies in the iodine-Pt(111) interaction, A. Tkatchenko, N. Batina, A. Cedillo y M. Galván, Surf. Sci. 581, 58 (2005).
} 
previos de primeros principios se han concentrado en la adsorción del yodo en el sitio $f$ de la superficie $\mathrm{Pt}(111)$ [84]. Sin embargo, creemos importante el estudio más exhaustivo de la interacción, por lo que se escogieron cuatro sitios distintos mostrados en la Figura 1.1.

Para estudiar la transferencia de carga entre el átomo de yodo y la superficie, se utiliza la partición de Hirshfeld de átomos en moléculas [85]. Una racionalización reciente de Nalewajski y Parr proporciona un sentido claro a esta partición desde el punto de vista de la teoría de la información $[86,87]$.

\subsubsection{Partición de Hirshfeld [84]}

La densidad electrónica de un átomo de yodo adsorbido, $\rho_{I S}(r)$, se representa como suma de dos densidades de fragmentos, $\rho_{I}(r)$ y $\rho_{S}(r)$, con números de electrones $N_{I}$ y $N_{S}$, respectivamente. Estos números en general difieren del número de electrones de los fragmentos aislados, $N_{I}^{0}$ y $N_{S}^{0}$, pero la suma de ellos da el número total correcto de electrones: $N_{I}+N_{S}$ $=N_{I S}^{0}=N_{I}^{0}+N_{S}^{0}$. En este contexto, la densidad de referencia se define como:

$$
\rho_{I S}^{0}=\rho_{S}^{0}+\rho_{I}^{0}
$$

donde $\rho_{S}^{0}$ y $\rho_{I}^{0}$ son las densidades de los fragmentos aislados.

La partición de Hirshfeld se escribe como:

$$
\begin{array}{r}
\rho_{\alpha}(r)=w_{\alpha}(r) \rho_{I S}(r), \\
w_{\alpha}(r)=\frac{\rho_{\alpha}^{0}(r)}{\rho_{I S}^{0}(r)} ; \alpha=I, S .
\end{array}
$$

Las funciones $w_{\alpha}$ son los factores que determinan la contribución relativa del fragmento (átomo adsorbido o superficie) $\alpha$. Los fragmentos definidos por la partición de Hirshfeld son regiones abiertas con cargas efectivas generalmente distintas de los fragmentos separados. 
Estas diferencias tienen origen en la transferencia de carga debido a la formación de enlaces químicos. Desde el punto de vista práctico se ha demostrado que el método de Hirshfeld permite medir transferencia de carga de una forma que se puede trasladar de un sistema a otro y tiene dependencia muy baja respecto a la base utilizada para el cálculo de la estructura electrónica $[88,89]$. En el caso particular de los sólidos, la partición de Hirshfeld se puede obtener por medio de integraciones de las densidades electrónicas en la celda unitaria.

\subsubsection{Modelo de superficie}

Los modelos de superficie empleados en esta sección son losas de cinco capas de cuatro o nueve átomos de platino perpendiculares a la cara (111) del cristal. Los átomos de yodo se adsorben por ambos lados de la superficie para eliminar el momento dipolar en la celda unitaria. Estos modelos corresponden a celdas $(2 \times 2)$ y $(3 \times 3)$ con grado de cobertura $1 / 4$ y $1 / 9$, respectivamente. Es importante mencionar que aún en la celda $(2 \times 2)$ la distancia I-I es dos veces más grande que el radio de van der Waals del átomo de yodo, el cual es alrededor de $2.2 \AA$, indicando que se espera una interacción débil en la monocapa para ambos modelos. La estructura electrónica se calcula con el formalismo de Kohn-Sham [90] utilizando LDA (local density approximation) y GGA-PBE (generalized gradient approximation en la parametrización de Perdew-Burke-Ernzerhoff) como funcionales de intercambio y correlación [91]. Las ecuaciones de Kohn-Sham fueron resueltas, utilizando una expansión en ondas planas truncada a partir de 40 Ry, en el programa DFT++ [92]. Los electrones internos de los átomos no se trataron explícitamente, sustituyendo estos por un pseudopotencial con conservación de la norma. Estos pseudopotenciales se generaron con el esquema de Trouillier-Martins [74] para cada tipo de funcional utilizado.

Es un hecho conocido que LDA proporciona mejores geometrías, mientras que las energías son mejores con el uso de GGA [93]. En concordancia con este hecho, el parámetro de malla para el bulto y la superficie (111) de Pt tienen un mejor acuerdo con el experimento cuando 
se utiliza LDA [94]. Para el caso de GGA, el parámetro de malla se sobreestima ligeramente por un $2 \%$. Los valores optimizados de parámetro de bulto utilizados en este estudio son: $3.92 \AA$ para LDA y $3.99 \AA$ para GGA. El uso de 8 y 4 puntos $k$ en la primera zona irreducible de Brillouin para las celdas $(2 \times 2)$ y $(3 \times 3)$, respectivamente, se determinó como apropiado para una convergencia de un millihartree en la energía total. La supercelda utilizada incluye una región de vacío equivalente a 6 capas de átomos de platino. El truncamiento de base de 40 Ry asegura una convergencia en la energía mejor que el logrado para el conjunto de puntos $k$. La geometría del sistema I-Pt(111) con un átomo de yodo adsorbido en los sitios $f, h, b$ y $a$ se relajó en los niveles de teoría LDA y GGA-PBE. Las fuerzas sobre los átomos en la celda unitaria se redujeron a $10^{-3}$ hartree/bohr. Para calcular las cargas efectivas de los fragmentos definidos por la Ecuación 3.4, se integraron las densidades de los fragmentos la misma malla numérica que se usa para calcular la densidad en el código DFT++. Aparte del cálculo de energía de un átomo de yodo separado, el cual se hizo utilizando el método de espín polarizado, todos los demás cálculos se hicieron con la metodología de espín restringido. Para el cálculo del átomo separado las características de la celda unitaria, el conjunto de puntos $k$ y el truncamiento de la base fueron los mismas que los del modelo de la superficie completa.

\subsubsection{Resultados}

La Tabla 3.1 muestra las distancias de equilibrio yodo-superficie, la carga sobre el átomo de yodo y las energías de adsorción, definidas como la diferencia entre la energía del sistema completo yodo-superficie y la energía de los fragmentos separados (yodo y superficie), para los sitios $f, h, b$ y $a$. Un hecho importante es que por definición energías de adsorción mayores indican una interacción más favorable. En relación a la distancia yodo-superficie, se puede concluir que los cálculos LDA están en buen acuerdo con los valores experimentales [80-82]. En todos los casos, la distancia de equilibrio $Z$ se calculó con respecto a la primera capa 
Tabla 3.1: La carga sobre el átomo de yodo $\left(q_{I}\right)$, distancia de equilibrio $(d)$ y energías de adsorción $\left(E_{a d s}\right)$ para distintos sitios en la superficie $\mathrm{Pt}(111)$. Las distancias se reportan en $\AA$ y las energías en $\mathrm{kcal} / \mathrm{mol}$. Las últimas cuatro columnas corresponden a cálculos GGA.

\begin{tabular}{lllllll}
\hline Sitio & $d[(2 \times 2) \mathrm{LDA}]$ & $d[(2 \times 2) \mathrm{GGA}]$ & $q_{I}[(2 \times 2)]$ & $q_{I}[(3 \times 3)]$ & $E_{\text {ads }}[(2 \times 2)]$ & $E_{\text {ads }}[(3 \times 3)]$ \\
\hline$f$ & $2.14(2.09-2.17)^{a}$ & 2.19 & 0.08 & 0.13 & 61.3 & $64.5(61.0)^{b}$ \\
$h$ & 2.15 & 2.20 & 0.08 & 0.12 & 60.9 & 64.1 \\
$b$ & 2.23 & 2.30 & 0.05 & 0.10 & 59.3 & 60.6 \\
$a$ & 2.53 & 2.59 & -0.02 & -0.02 & 51.7 & 52.2 \\
\hline
\end{tabular}

${ }^{a}$ Los valores experimentales fueron tomados de Ref. [81]

${ }^{b}$ Valor experimental extrapolado a $0 \mathrm{~K}$; Ref. [82]

de la superficie. El orden en la distancia perpendicular para distintos sitios de adsorción es el esperado: $f \approx h<b<a$ (vea Figura 1.1). Las distancias optimizadas GGA son más largas que LDA, lo cual es un hecho conocido para estos dos funcionales de intercambio y correlación. Sin embargo, el orden es el mismo en ambos casos. Las energías de adsorción obtenidas con GGA, relajando el parámetro de malla de la superficie y la geometría dentro de la celda unitaria muestran un comportamiento estándar, el cual es el inverso de las distancias de equilibrio. En relación a las energías de adsorción es importante mencionar que los cálculos anteriores reportados en la literatura, utilizando un modelo de cúmulos finitos para la superficie de platino [84], se desvían por más de $50 \mathrm{kcal} / \mathrm{mol}(120 \mathrm{kcal} / \mathrm{mol})$, mientras que nuestra estimación para el caso del sitio $f$ en la celda $(3 \times 3)$ difiere solamente $4 \mathrm{kcal} / \mathrm{mol}$ (65 $\mathrm{kcal} / \mathrm{mol}$ ) del valor experimental extrapolado a $0 \mathrm{~K}$ [82]. Estos resultados demuestran que el modelo de adsorción y el procedimiento de cálculo empleado en este trabajo es el más preciso utilizado hasta ahora para la descripción del sistema I-Pt(111).

Para determinar la magnitud y el mecanismo de la transferencia de carga entre el átomo de yodo adsorbido y la superficie, se pueden dividir los efectos en globales y locales. Los primeros describen los efectos totales o las cargas de los fragmentos definidos en la sección 3.2.1, que se calculan como la diferencia $N_{\alpha}-N_{\alpha}^{0}$; la cantidad $N_{\alpha}$ se calcula a partir de la integración sobre las densidades de fragmentos de Hirshfeld, $\rho_{\alpha}(r)$ en la celda unitaria. Estas diferencias 
se reportan en la Tabla 3.1 para el caso de GGA; LDA da resultados similares. En contraste con estudios teóricos previos, se puede concluir que existe una transferencia electrónica hacia la superficie $\mathrm{Pt}(111)$ cuando el yodo se adsorbe sobre ésta, excepto en el caso del sitio a, para el cual la transferencia de carga es mínima. Se observa una clara tendencia en la transferencia de carga: a la energía de adsorción más grande le corresponde una transferencia de carga mayor. Para el sitio $a$, el valor absoluto de la transferencia es el menor y se puede observar una tendencia de orden decreciente desde el sitio $f$ hasta el sitio $a$, pasando por los sitios $h$ y $b$. Existe evidencia experimental indirecta de que el átomo de yodo permanece con una carga relativamente pequeña al ser adsorbido en la superficie $\mathrm{Pt}(111)$ [80]. Este hecho se puede relacionar con nuestros hallazgos y se puede racionalizar por medio de valores de la energía de adsorción para distintos sitios. Sin embargo, hay que notar que no es posible hacer una comparación directa de transferencia de carga obtenida en este trabajo con los valores obtenidos por medio de un experimento, debido a las diversas contribuciones a la transferencia de carga en experimentos de electroquímica.

Por otro lado, los efectos locales de transferencia de carga se pueden analizar por medio de las diferencias de densidad, $\Delta \rho(r)=\rho_{I S}(r)-\rho_{I S}^{0}(r)$. Los valores positivos de esta diferencia indican regiones con acumulación de carga debido a la interacción yodo-superficie, mientras que las regiones negativas indican lo contrario. Las Figuras 3.1 y 3.2 muestran la densidad total, $\rho_{I S}(r)$ y las diferencias de densidad para los sitios $f$ y $a$, respectivamente. En el caso del sitio $f$, el enlace I-Pt se puede observar en la diferencia de densidad como una región verde tenue entre el átomo de yodo y la primera capa de la superficie; para el caso del sitio $a$, este enlace se representa con una región azul tenue. Las diferencias de densidad para ambos sitios muestran efectos fuertes sobre la primera capa de platinos. Así mismo, para ambos sitios de adsorción existen regiones de disminución de carga (azul-verde) con apariencia de un orbital $p$ alrededor del átomo de platino; esta región posee otra región acompañante (rojaamarilla) con apariencia de un orbital $d$. Esta combinación de regiones sugiere una especie 

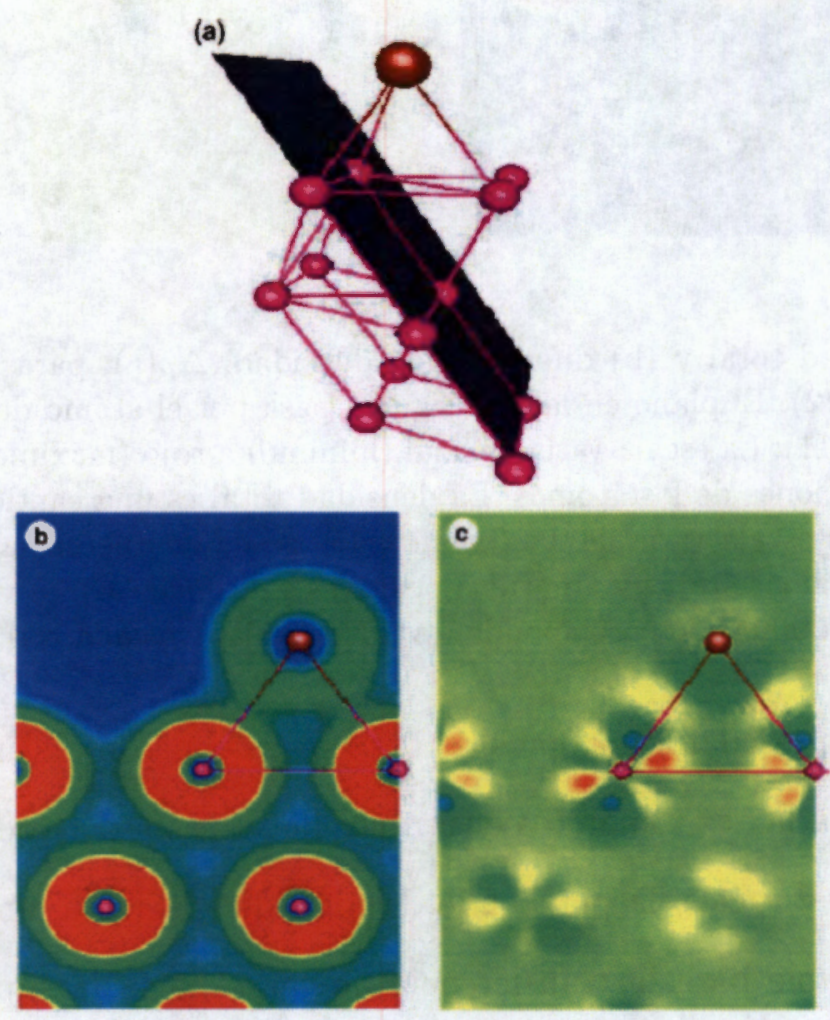

Figura 3.1: (a) Celda unitaria, (b) densidad total, (c) diferencia de densidad, $\Delta \rho(r)$, para el sitio $f$ del sistema I-Pt(111) en la celda $(2 \times 2)$. Solamente se muestran tres de las cinco capas de la superficie. El plano en las dos figuras pasa por el átomo de yodo y dos átomos de platino como se indica en el panel (a); Uno tiene que recordar que la copia periódica del átomo de yodo en la celda vecina a la izquierda se situará sobre el plano. La escala varía de azul (mínimo) a rojo (máximo); las esferas de color púrpura indican las posiciones de los átomos. La densidad total es una cantidad positiva definida, por lo que en este caso la escala varía de 0 a 0.39 u.a. La escala de diferencia de densidad varia de -0.056 a 0.027 u.a. con regiones rojas y amarillas positivas y regiones azules y verdes negativas. 

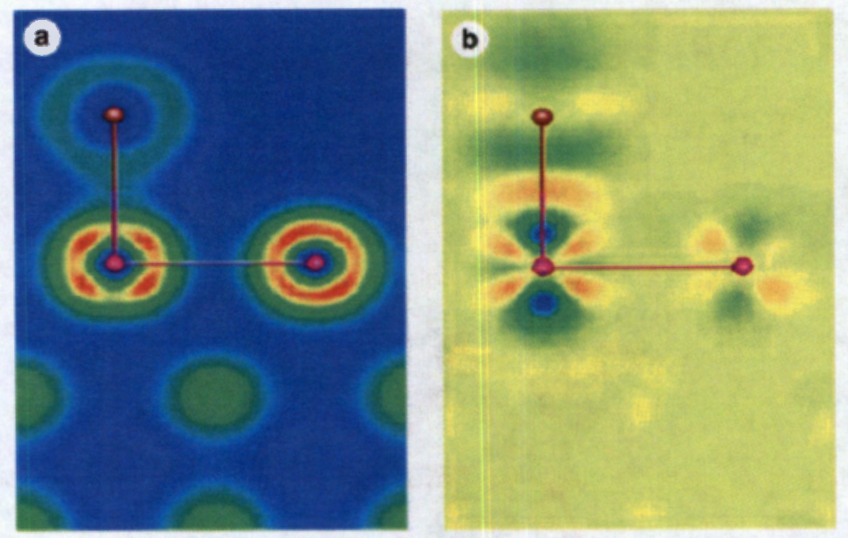

Figura 3.2: (a) Densidad total y (b) diferencia de densidad, $\Delta \rho(r)$, para el sitio a del sistema I-Pt(111) en la celda (2×2). El plano en las dos figuras pasa por el átomo de yodo y un átomo de platino en la dirección (111); La escala varía de azul (mínimo) a rojo (máximo); las esferas de color púrpura indican las posiciones de los átomos. La densidad total es una cantidad positiva definida, por lo que en este caso la escala varia de 0 a 0.39 u.a. La escala de diferencia de densidad varía de -0.078 a 0.029 u.a. con regiones rojas y amarillas positivas y regiones azules y verdes negativas. En este caso el color de fondo amarillo corresponde a valores de la densidad cercanos a cero.

de retrocoordinación, en la cual los átomos de la primera capa de platino donan electrones a la región de enlace y a la vez toman los electrones de valencia del yodo que finalmente se localizan en los estados $d$ vacíos. Dependiendo del sitio de adsorción, ambos efectos pueden crear un balance favorable para la transferencia de carga hacia la superficie para el caso de los sitios $f, h$ y $b$ o resultar en una contribución insignificante para el sitio $a$. El orden de magnitud de las energías de adsorción indica una interacción fuerte entre el yodo y la superficie. Asimismo, se puede concluir que la interacción para el sitio a es la menos iónica y el carácter iónico posee el siguiente orden: $f \approx h>b>a$.

Comparando los resultados obtenidos en las celdas $(2 \times 2)$ y $(3 \times 3)$, se puede decir que, mientras que la transferencia de carga para el sitio a no depende del grado de cobertura, hay un aumento en la cantidad de carga transferida a la superficie para los otros tres sitios cuando el grado de cobertura es reducido; la equivalencia en la transferencia de carga entre los sitios $f$ y $h$ en la celda $(2 \times 2)$ se pierde ligeramente para el caso de $(3 \times 3)$. Es importante 
notar que para ambas celdas, la disminución en el grado de cobertura se relaciona con una transferencia de carga mayor del yodo a la superficie para los sitios $f, h$ y $b$. Esto se puede explicar en términos de la máxima cantidad de carga que puede donar la monocapa de yodo. El aumento en el grado de cobertura (más átomos de yodo por unidad de área) significaría una reducción en la carga transferida. Un comportamiento similar se observa para las energías de adsorción: cuando nos movemos de la celda $(2 \times 2)$ a la celda $(3 \times 3)$, la similitud entre los sitios $f$ y $h$ se mantiene dentro de un rango de $1 \mathrm{kcal} / \mathrm{mol}$. Aun así, se puede notar que la diferencia en la energía de adsorción para el sitio $f$ entre la celda $(2 \times 2)$ y $(3 \times 3)$ difiere en $4 \mathrm{kcal} / \mathrm{mol}$, lo que se puede explicar por una relajación de geometría mayor en el caso de la celda $(3 \times 3)$. Sin embargo, para ambos modelos, el sitio $a$ es el que se caracteriza por tener menor estabilidad.

Para verificar el efecto que tiene el dipolo inducido por un modelo de adsorción de un solo lado, repetimos los cálculos para el caso del sitio $f$ en la celda $(3 \times 3)$ con el átomo de yodo adsorbido en un solo lado. La transferencia de carga cambia en el orden de 0.001 electrones y la energía de adsorción en el orden de $0.3 \mathrm{kcal} / \mathrm{mol}$. Ambos valores no son significativos ya que están dentro del margen de error del nivel de teoría empleado.

\subsubsection{Discusión}

En contraste con nuestros resultados, todos los estudios previos han reportado una transferencia de carga desde la superficie Pt al átomo de yodo. Aún más importante es el hecho de que las energías de adsorción de todos los estudios previos están alejados $60 \mathrm{kcal} / \mathrm{mol} \mathrm{de}$ los resultados experimentales [84]. A diferencia de lo anterior, la distancia yodo-superficie para el sitio $f$ encontrada en este trabajo está en concordancia con los datos teóricos y experimentales previos. Es importante mencionar que todos los estudios anteriores han empleado el modelo de cúmulos finitos para describir la superficie de platino, con un tamaño de cúmulo de 3 a 12 átomos [83, 84]. Para analizar el impacto de usar un modelo de cúmulo finito 
en comparación con el modelo periódico utilizado en este trabajo, se hicieron cálculos con un cúmulo de 10 átomos y el átomo de yodo adsorbido en el sitio $f$. Se encontró una alta contaminación de espín en los cálculos con un conjunto de base localizado. Con el modelo periódico, solamente el cálculo de un yodo aislado en la celda requiere el método de espín polarizado. Otra fuente de errores mencionados arriba puede relacionarse con el hecho de que los cálculos con modelos finitos corresponden a grados de cobertura muy bajos. La diferencia en energías de adsorción entre el modelo finito y nuestro trabajo puede ser el resultado de una combinación de estos efectos. Los resultados experimentales siempre implican un grado de cobertura definido, el cual podría estar más cercano al modelo empleado en este trabajo que al límite del modelo finito. Así, el acuerdo de nuestros resultados con las energías de adsorción experimentales obtenidas por medio de la técnica de desorción programada por temperatura (TPD) y geometrías obtenidas por medio de difracción de electrones de bajas energías (LEED) indica que el modelo periódico es el más adecuado para estudiar este tipo de sistemas.

En los primeros estudios experimentales realizados sobre I-Pt(111), uno puede encontrar mucha especulación sobre la naturaleza covalente del enlace yodo-superficie [37,59]. Nuestros resultados confirman la covalencia de modo definitivo. Asímismo, de nuestro análisis de diferencias de densidad es claro que la interacción entre el yodo y la superficie Pt no se puede asociar a fuerzas de van der Waals exclusivamente.

El esquema de partición de Hirshfeld utilizado en este trabajo proporciona resultados que son cualitativamente equivalentes a otros esquemas, como el análisis de población de Mulliken [95] o el esquema de átomos en moléculas (AIM) de Bader [96]. Las transferencias de carga obtenidas con el esquema de Hirshfeld son generalmente mas pequeñas que las dadas por el análisis de población estándar; sin embargo, con el método de Hirshfeld se obtiene una buena descripción cualitativa que no depende del conjunto de base utilizado. Este esquema de partición se ha utilizado satisfactoriamente en estudios sobre interacciones intra-moleculares, 
pero creemos que puede ser de gran ayuda para estudiar procesos de transferencia de carga en la ciencia de superficies, particularmente cuando se usan conjuntos de bases deslocalizados (como ondas planas). En nuestro caso particular, la partición de Hirshfeld da números de transferencia de carga consistentes con la tendencia de energías de adsorción.

La diferencia de densidad $\Delta \rho(r)=\rho_{I S}(r)-\rho_{I S}^{0}(r)$ es la función que se utiliza generalmente para determinar las transferencias de carga en estudios de estructuras electrónica de la materia condensada [5]. Partiendo de las densidades de fragmentos de Hirshfeld se observa que la diferencia de densidad se puede descomponer a su vez en diferencias de densidades de fragmentos de Hirshfeld, uno para la superficie y otro para el adsorbato: $\Delta \rho(r)=\left(\rho_{S}(r)-\right.$ $\left.\rho_{S}^{0}(r)\right)+\left(\rho_{I}(r)-\rho_{I}^{0}(r)\right)$. Es interesante ver de la Figura 3.3 que, afuera de la región del enlace, la diferencia de densidad es igual a una de éstas diferencias de fragmentos, sin embargo en la zona de enlace ambos componentes contribuyen en acuerdo con la naturaleza covalente del enlace químico. Como $\Delta \rho(r)$ tiene que integrar a cero, en este caso los dos fragmentos integran a valores opuestos: la transferencia de carga correspondiente.

Existe evidencia experimental indirecta de que los aniones de yodo permanecen con poca carga al adsorberse sobre la superficie $\mathrm{Pt}(111)$ [37], lo que indica que la carga se transfiere desde el yodo hacia la superficie $\mathrm{Pt}(111)$, en concordancia con nuestros resultados teóricos. Asimismo, se obtiene un acuerdo cuantitativo en las energías de adsorción con datos experimentales previos, por lo que consideramos que nuestros resultados contribuyen a una mejor interpretación de experimentos en sistemas sustrato-adsorbato a nivel atómico.

En conclusión, en estas últimas secciones utilizamos el formalismo de sistema periódico en la TFD para describir la adsorción de yodo en la superficie Pt(111). La transferencia de carga y las energías de adsorción obtenidas en este trabajo están de acuerdo con los valores experimentales. También se propone el uso de partición de Hirshfeld para analizar la transferencia de carga en sistemas sustrato-adsorbato y ésta se aplica con éxito para la descripción del sistema I-Pt(111). Los resultados indican que el uso de esta partición 


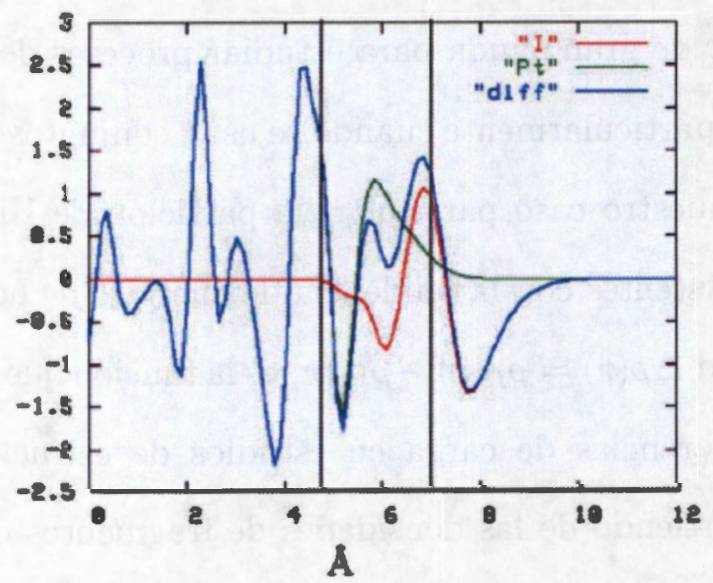

Figura 3.3: La diferencia de densidad de fragmento de Hirshfeld para yodo $\rho_{I}(r)-\rho_{I}^{0}(r)(\mathrm{I})$, la diferencia de densidad de fragmento de Hirshfeld para la superficie Pt $\rho_{S}(r)-\rho_{S}^{0}(r)(\mathrm{Pt})$, obtenidos para el sitio $f$ de la superficie en la celda $(2 \times 2)$, y la diferencia de densidad $\Delta \rho(r)$ (diff) integrada sobre el plano $x y$. El eje $x$ marca la distancia vertical desde la tercera capa de la superficie. El yodo esta situado en $z=6.67 \AA$. La región del enlace (entre la primera capa de superficie y el átomo de yodo) está marcada con líneas negras verticales.

puede ser promisorio para estudios posteriores en el campo de la ciencia de superficies y materia condensada. Por otro lado, se demuestra que los modelos de cúmulos finitos utilizados anteriormente no son adecuados para la descripción del sistema I-Pt(111).

\subsection{Estructuras ordenadas de I-Pt(111)}

En esta sección se analiza la formación de distintas estructuras ordenadas de yodo en la superficie Pt(111) por medio de cálculos de TFD. Entre los objetivos principales está la identificación de estructuras de mínima energía en las celdas en las que experimentalmente se han encontrado estructuras y obtener energías de adsorción que pueden ser útiles posteriormente en el ajuste de modelos para interacciones en la monocapa adsorbida de yodo. Por lo tanto, esta sección complementa los datos de energía y transferencia de carga para un solo átomo de yodo en $\mathrm{Pt}(111)$. 


\subsubsection{Metodología}

Se efectuó una búsqueda de puntos estacionarios en varias celdas de la superficie $\mathrm{Pt}(111)$ con distintos grados de cobertura. Se escogieron celdas en las cuales se sabe que existen estructuras estables a partir de algunos estudios experimentales previos: $(\sqrt{3} \times \sqrt{3}) R 30^{\circ}$, $(\sqrt{7} \times \sqrt{7}) R 19.11^{\circ}$ y $(3 \times 3)$ con grados de cobertura $1 / 3,3 / 7,4 / 9$, respectivamente. Se probaron distintas configuraciones iniciales en cada una de estas celdas, correspondientes a estructuras con menor y mayor altura promedio de los átomos de yodo, los cuales se determinaron de los estudios geométricos previos sobre el sistema I-Pt(111) [15, 16]. Es importante recordar que los modelos de las superficies mencionadas arriba contienen diferente cantidad de átomos de yodo por celda unitaria.

La estructura electrónica se calcula con el formalismo de Kohn-Sham [90] utilizando LDA y GGA-PBE como funcionales de intercambio y correlación [91,97]. Las ecuaciones de KohnSham fueron resueltas utilizando una expansión en ondas planas truncada a 40 Ry dentro del programa DFT++ [92]. Los electrones internos se reemplazaron por un pseudopotencial de tipo Troullier-Martins [74] y se diseñaron para cada tipo de funcional. Todas las energías en este trabajo se reportan a nivel de teoría GGA y las geometrías a nivel de teoría LDA, en donde para la descripción de la superficie se utiliza el parámetro de bulto optimizado y se realiza una relajación de geometría para cada tipo de funcional. Para las distintas celdas de la superficie $\mathrm{Pt}(111)$ usadas en este trabajo, el grado de cobertura cambia desde $\theta=0.33$ a $\theta=0.44$, por lo que construímos dos pseudopotenciales para los átomos de yodo, uno con 7 otro con 17 electrones de valencia. Esto se hace para probar la influencia de electrones internos del átomo de yodo en la descripción energética de monocapas con alto grado de cobertura $\theta=0.44$. Para el caso del pseudopotencial con 17 electrones de valencia, la energía de truncamiento se ajustó a 60 Ry para describir correctamente el orbital $4 d$ del yodo.

Se encontró que 8 puntos $k$ para la estructura $(\sqrt{3} \times \sqrt{3}) R 30^{\circ}$ y 4 puntos $k$ para las estructuras $(\sqrt{7} \times \sqrt{7}) R 19.11^{\circ}$ y $(3 \times 3)$ son suficientes para la convergencia de las diferencias 

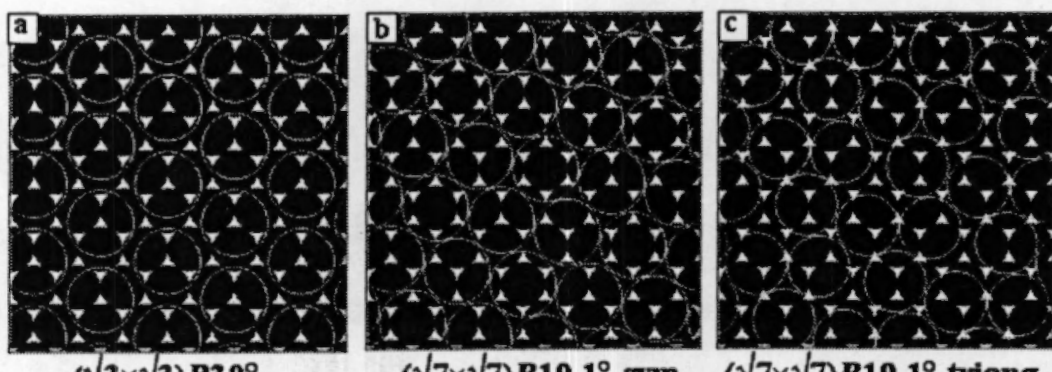

$(\sqrt{3} \times \sqrt{3}) R 30^{\circ}$

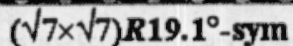

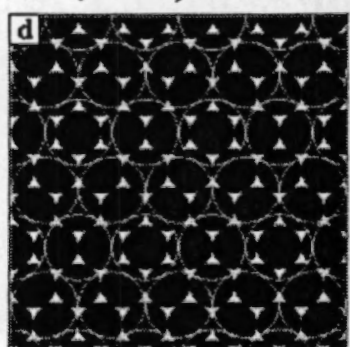

$(3 \times 3)-5 \mathrm{~mm}$

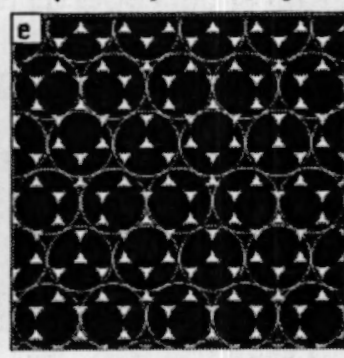

(3x3)-asym
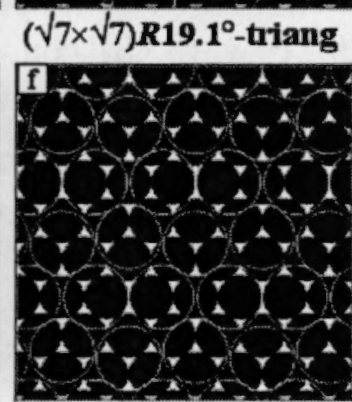

(3)3)-xigrag

Figura 3.4: Las estructuras estables para las distintas celdas escogidas en esta sección. El nombre de cada estructura se especifica abajo del modelo.

Tabla 3.2: Energías de adsorción (en $\mathrm{kcal} / \mathrm{mol}$ ) para distintos sitios en las celdas $(3 \times 3)$ y $(2 \times 2)$.

\begin{tabular}{lcc}
\hline Sitio & $(2 \times 2)$ & $(\sqrt{3} \times \sqrt{3}) R 30^{\circ}$ \\
\hline$f$ & 61.3 & 59.0 \\
$h$ & 60.9 & 58.9 \\
$b$ & 59.3 & 57.7 \\
$a$ & 51.7 & 49.7 \\
\hline
\end{tabular}

de energía. El parámetro de malla de platino se tomó como $3.99 \AA$ para GGA y $3.92 \AA$ para LDA y las fuerzas sobre los átomos en la celda unitaria (la capa de yodo y dos capas más cercanas de la superficie) se relajaron hasta que las fuerzas se redujeron a $10^{-3}$ hartree/bohr. 


\subsubsection{Resultados}

\section{Energías de adsorción}

Para comparar las energías de adsorción con los resultados de la sección anterior, se hicieron cálculos para los sitios $f, h, b$ y $a$ en la celda $(\sqrt{3} \times \sqrt{3}) R 30^{\circ}$ (Fig. 3.4a) con un átomo por celda unitaria. La Tabla 3.2 muestra las energías de adsorción de yodo en estos sitios para las celda $(2 \times 2)$ y $(\sqrt{3} \times \sqrt{3}) R 30^{\circ}$. La distancia entre los átomos de yodo en estas celdas es de $5.64 \AA$ y $4.89 \AA$, respectivamente. Se ve que las energías de adsorción son más bajas para el caso de la celda $(\sqrt{3} \times \sqrt{3}) R 30^{\circ}$, indicando una posible interacción repulsiva lateral entre los átomos de yodo.

En la celda $(\sqrt{7} \times \sqrt{7}) R 19.11^{\circ}$, se pudieron identificar dos estructuras distintas, las cuales se muestran en la Fig. 3.4. Utilizaremos los nombres $(\sqrt{7} \times \sqrt{7}) R 19.11^{\circ}$-sym y $(\sqrt{7} \times \sqrt{7}) R 19.11^{\circ}$-triang para las estructuras en la Fig. $3.4 \mathrm{~b}$ y $3.4 \mathrm{c}$, respectivamente. La estructura $(\sqrt{7} \times \sqrt{7}) R 19.11^{\circ}$-sym fue observada experimentalmente y, en consecuencia, es la más estable en la celda $(\sqrt{7} \times \sqrt{7}) R 19.11^{\circ}$, con una energía de adsorción de $56.4 \mathrm{kcal} / \mathrm{mol}$ por átomo de yodo. Por otro lado, la estructura $(\sqrt{7} \times \sqrt{7}) R 19.11^{\circ}$-triang posee una simetría muy interesante, con todos los átomos de yodo cercanos al sitio $b$. La distancia entre los átomos vecinos en la estructura $(\sqrt{7} \times \sqrt{7}) R 19.11^{\circ}$-triang es menor que en la estructura $(\sqrt{7} \times \sqrt{7}) R 19.11^{\circ}$-sym que posee una simetría hexagonal perfecta, lo que puede indicar una mayor repulsión en la monocapa y explicar una energía de adsorción de $54.6 \mathrm{kcal} / \mathrm{mol}$ por átomo de yodo en la primera, $1.8 \mathrm{kcal} / \mathrm{mol}$ menor que en la segunda.

Se pudieron identificar tres estructuras distintas en la celda $(3 \times 3)$ : $(3 \times 3)$-sym, $(3 \times 3)$ asym y (3×3)-zigzag, las cuales se muestran en la Fig. 3.4(d-f). Las dos primera estructuras se han observado en distintos experimentos $[37,59,80,98]$. Es interesante notar que la estructura (3×3)-zigzag tiene una energía de adsorción de $53.1 \mathrm{kcal} / \mathrm{mol}$, un valor que se encuentra entre la energía de adsorción de $(3 \times 3)$-sym $(54.5 \mathrm{kcal} / \mathrm{mol})$ y $(3 \times 3)$-asym $(52.2 \mathrm{kcal} / \mathrm{mol})$. Vale 
Tabla 3.3: Energías de adsorción (en $\mathrm{kcal} / \mathrm{mol}$ ) para distintas estructuras en el sistema I-Pt(111).

\begin{tabular}{lcc}
\hline Estructura & Energía de adsorción & $\theta$ \\
\hline$(2 \times 2)$-fcc & 61.3 & $1 / 4$ \\
$(\sqrt{3} \times \sqrt{3}) R 30^{\circ}$-fcc & 59.0 & $1 / 3$ \\
$(\sqrt{7} \times \sqrt{7}) R 19.11^{\circ}$-sym & 56.4 & $3 / 7$ \\
$(\sqrt{7} \times \sqrt{7}) R 19.11^{\circ}$-triang & 54.6 & $3 / 7$ \\
$(3 \times 3)$-sym & 54.5 & $4 / 9$ \\
$(3 \times 3)$-asym & 52.2 & $4 / 9$ \\
$(3 \times 3)$-zigzag & 53.1 & $4 / 9$ \\
\hline
\end{tabular}

la pena resaltar que los arreglos geométricos de las tres estructuras encontradas en la celda $(3 \times 3)$ son cualitativamente distintos. La estructura con la simetría equivalente a $(3 \times 3)$-zigzag se encontró en el estudio experimental del sistema $\mathrm{Br}-\mathrm{Pt}(111)$ por Orts y colaboradores [99].

En la Tabla 3.3 se resumen los datos de energías de adsorción para todas las estructuras de mímima energía. Más adelante, se mostrará que la interacción lateral en la monocapa adsorbida de yodo es repulsiva, este hecho se confirma por las energías de adsorción en las celdas $(2 \times 2)$ y $(\sqrt{3} \times \sqrt{3}) R 30^{\circ}$ y el análisis de las estructuras en la celda $(\sqrt{7} \times \sqrt{7}) R 19.11^{\circ}$.

En este punto es interesante comparar las diferencias en energías de adsorción entre distintas estructuras que se obtienen en este estudio y encontradas por medio de la técnica TPD [82]. Este estudio experimental reporta las entalpías de desorción extrapoladas a 0 K. En el caso del yodo en el límite de cobertura cero, reporta un valor de $61 \mathrm{kcal} / \mathrm{mol}$. Si comparamos este valor con la energía de adsorción de un solo átomo de yodo en el sitio $f$ en la celda $(3 \times 3)$ [Tabla 3.1], encontramos un buen acuerdo con los datos experimentales (64 $\mathrm{kcal} / \mathrm{mol}$ contra $61 \mathrm{kcal} / \mathrm{mol}$ ), con una diferencia de $3 \mathrm{kcal} / \mathrm{mol}$. Experimentalmente, la diferencia entre la estructura $(\sqrt{3} \times \sqrt{3}) R 30^{\circ}$ (un átomo por celda unitaria) y cobertura cero es de $8.6 \mathrm{kcal} / \mathrm{mol}$ [82]. Nuestros cálculos arrojan un valor de $5.5 \mathrm{kcal} / \mathrm{mol}$. Así mismo, la diferencia experimental entre las estructuras $(\sqrt{3} \times \sqrt{3}) R 30^{\circ}$ y $(\sqrt{7} \times \sqrt{7}) R 19.11^{\circ}$-sym es de $4.3 \mathrm{kcal} / \mathrm{mol}$, mientras que nosotros obtenemos $2.6 \mathrm{kcal} / \mathrm{mol}$. Las barras de error 
experimentales son de $10 \mathrm{kcal} / \mathrm{mol}$ [82], por lo que creemos que el acuerdo con el experimento es aceptable.

Para estudiar el efecto de los electrones internos del átomo de yodo sobre las energías de adsorción, se hicieron cálculos de distintas estructuras con el pseudopotencial que contiene 17 electrones de valencia para el yodo con una energía de corte de $60 \mathrm{Ry}$. Con esto, las energías de adsorción se redujeron en alrededor de $1 \mathrm{kcal} / \mathrm{mol}$ para todas las estructuras. Esto indica que el pseudopotencial con 7 electrones de valencia es suficientemente bueno para tratar el sistema I-Pt(111) con grado de cobertura $\theta<\frac{4}{9}$.

\section{Geometrías de adsorción}

Saidy y colaboradores hicieron un estudio LEED sobre distintas estructuras en el sistema I-Pt(111) [81]. Es interesante contrastar las distancias de equilibrio yodo-Pt(111) obtenidas con LDA con los valores experimentales. La distancia perpendicular de enlace I-Pt que obtenemos es de $2.17 \AA$ para la estructura $(\sqrt{3} \times \sqrt{3}) R 30^{\circ}$-fcc, en perfecto acuerdo con los datos experimentales $(2.17 \AA)$. La distancia lateral entre los tres átomos de platino que participan en el enlace con el átomo de yodo adsorbido se incrementa desde $2.885 \AA$ a 2.890 $\AA$. La diferencia en posiciónes verticales de platino en la primera capa de superficie es de $0.02 \AA$. En el caso de la estructura $(\sqrt{7} \times \sqrt{7}) R 19.11^{\circ}$-sym, nuestros resultados indican que el átomo de yodo en el sitio $f$ muestra una longitud de enlace de $2.12 \AA$, en el sitio $h$ de $2.19 \AA$ y en el sitio $a$ de $2.72 \AA$. Esto da una corrugación ${ }^{2}$ de $0.6 \AA$ en la capa de yodo, similar al estudio LEED experimental ( $0.53 \AA$ ), aunque nuestra diferencia entre el sitio $f$ y $h$ es de $0.07 \AA$, mayor que los $0.03 \AA$ encontrado experimentalmente. En el caso de la celda $(3 \times 3)$, los datos experimentales no son confiables, ya que se demostró en diferentes estudios $[59,80,98]$, que existe una mezcla de simetrías sym y asym en la celda $(3 \times 3)$. Para (3×3)-sym, encontramos que una longitud de enlace de $2.77 \AA$ para el sitio $a, \mathrm{y}$ un valor

${ }^{a}$ En este caso la palabra corrugación se refiere a la diferencia en la distancia de equilibrio entre los sitios $a$ y $f$. Estos sitios corresponden al menos estable y al más estable, respectivamente. 
Tabla 3.4: Energías de relajación de la superficie $\mathrm{Pt}(111)$ (en kcal/mol) para distintas estructuras en el sistema I-Pt(111).

\begin{tabular}{lc}
\hline Estructura & Energía de relajación \\
\hline$(3 \times 3)$-fcc & 5.2 \\
$(2 \times 2)$-fcc & 1.9 \\
$(\sqrt{3} \times \sqrt{3}) R 30^{\circ}$-fcc & 1.8 \\
$(2 \times 2)$-atop & 0.3 \\
$(2 \times 2)$-bridge & 1.2 \\
$(\sqrt{7} \times \sqrt{7}) R 19.11^{\circ}$-sym & 1.6 \\
\hline
\end{tabular}

promedio de $2.27 \AA$ para los sitios $b$. La corrugación determinada es de $0.5 \AA$, un poco menor que en la estructura $(\sqrt{7} \times \sqrt{7}) R 19.11^{\circ}$-sym, lo que es de esperarse, ya que en la última estructura existen átomos de yodo en los sitios $f$ y a (el más y el menos estable), mientras que en la estructura $(3 \times 3)$-sym solamente existen sitios $b$ y $a$. Para la estructura $(3 \times 3)$-asym, existen tres distintos sitios: $f, h$ y sitio asimétrico; el átomo de yodo en el sitio $f$ tiene una longitud de enlace de $2.12 \AA$ y en el sitio asimétrico ésta es de $2.47 \AA$.

Efecto de la relajación de superficie sobre las energías de adsorción

Es interesante separar varias contribuciones a la energía total de adsorción para distintas estructuras en el sistema I-Pt(111). Un posible efecto es la relajación geométrica de la superficie inducida por la adsorción de una monocapa. Para analizar lo anterior en el caso I-Pt(111), se hicieron relajaciones estructurales de distintas monocapas de yodo con la superficie (111) congelada. Se determinó la energía de relajación de la superficie como $E_{\text {rel }}-E_{f i j a}$, donde $E_{\text {rel }}$ es la energía de adsorción con la superficie totalmente relajada (sin ninguna restricción) y $E_{f i j a}$ es la energía de adsorción con la superficie congelada utilizando el parámetro de bulto correspondiente a PBE-GGA. La Tabla 3.4 muestra las energías de relajación para distintas estructuras. Cuando la superficie se mantiene congelada, la estructura $(3 \times 3)$-fcc posee la misma energía de adsorción que la $(2 \times 2)$-fcc. Esto indica que la energía de adsorción mayor en el caso de la celda $(3 \times 3)$ se debe exclusivamente a la relajación de 
la superficie. Las energías de relajación para las estructuras $(2 \times 2)$-fcc y $(3 \times 3)$-fcc son 5.2 $\mathrm{kcal} / \mathrm{mol}$ y $1.9 \mathrm{kcal} / \mathrm{mol}$, respectivamente. Para estudiar el efecto de relajación de la superficie para el caso de estructuras con varios átomos por celda unitaria, se calculó éste para la estructura $(\sqrt{7} \times \sqrt{7}) R 19.11^{\circ}$-sym, la cual tiene átomos de yodo en los sitios $a, f$ y $h$. La energía de relajación que se obtuvo fue de $1.6 \mathrm{kcal} / \mathrm{mol}$ por átomo.

\subsubsection{Discusión}

Analizando los resultados de la sección previa, se mostrará que la interacción efectiva entre los átomos en la monocapa de yodo es repulsiva. Para esto, vamos a analizar las energías de adsorción en el caso de las celdas $(2 \times 2)$ y $(\sqrt{3} \times \sqrt{3}) R 30^{\circ}$. Para todos los sitios en la superficie $(a, b, f)$ la energía de adsorción disminuye mientras la distancia entre los átomos disminuye. Las mismas conclusiones se pueden obtener para el caso de $(\sqrt{7} \times \sqrt{7}) R 19.11^{\circ}$ con tres átomos por celda unitaria. Si se desprecia la interacción entre los átomos de yodo, la energía de adsorción se puede escribir como la suma de interacciones átomo-superficie para los tres átomos en la celda. En ese caso, la estructura $(\sqrt{7} \times \sqrt{7}) R 19.11^{\circ}$-triang sería más estable que la $(\sqrt{7} \times \sqrt{7}) R 19.11^{\circ}$-sym. Sin embargo, si observamos las energías de adsorción obtenidas con cálculos de TFD en la Tabla 3.3, vemos que la energía de adsorción por átomo de la estructura $(\sqrt{7} \times \sqrt{7}) R 19.11^{\circ}$-sym es menor en $1.8 \mathrm{kcal} / \mathrm{mol}$, lo que indica que la distancia más cercana entre los átomos en la estructura $(\sqrt{7} \times \sqrt{7}) R 19.11^{\circ}$-triang hace que la interacción entre éstos sea repulsiva.

También se pueden estudiar las tendencias en las energías de relajación de la superficie. Si tomamos la estructura $(\sqrt{7} \times \sqrt{7}) R 19.11^{\circ}$-sym, podemos ver que su energía de relajación de superficie puede ser descrita como una suma de energías de relajación para los tres sitios en la celda. Tomando como referencia las energías de relajación en la celda $(2 \times 2)$ (Tabla 3.4) y haciendo una suma sobre los sitios presentes en la estructura $(\sqrt{7} \times \sqrt{7}) R 19.11^{\circ}$-sym, se obtiene un valor de $1.3 \mathrm{kcal} / \mathrm{mol}$, cercano a $1.6 \mathrm{kcal} / \mathrm{mol}$ obtenido relajando la superficie 
sin restricciones. Aunque quisieramos extender esta extrapolación a otras estructuras, no se puede afirmar que siempre será precisa. Por ejemplo, para el caso de arreglos con simetría diferente de la hexagonal, como $(\sqrt{7} \times \sqrt{7}) R 19.11^{\circ}$-triang, los efectos de relajación se podrían desviar de una simple suma sobre átomos aislados.

Por otro lado, las geometrías y energías de adsorción en su mayoría están en concordancia con los datos experimentales donde estos existen. Por otro lado, la identificación de nuevas estructuras en las celdas $(\sqrt{7} \times \sqrt{7}) R 19.11^{\circ}$ y $(3 \times 3)$ indica que la superficie de energía potencial del sistema I-Pt(111) es compleja. 


\section{Capítulo 4}

\section{Análisis combinado del sistema}

\section{$(3 \times 3)-I-P t(111)$}

En este capítulo se estudia a detalle la superficie de energía potencial (SEP) del sistema (3×3)-I-Pt(111), con cuatro átomos de yodo en la celda unitaria. ${ }^{a}$ Para este propósito utilizamos metodología mixta de cálculos de TFD y simulaciones Monte Carlo (MC) para buscar mínimos locales en la celda unitaria.

\subsection{Planteamiento del problema}

La investigación de la superficie de energía potencial de distintos sistemas, como cúmulos, moléculas de interés biológico y vidrios ha sido de gran interés en los últimos años [3]. La disponibilidad de potenciales de interacción confiables ha permitido hacer un análisis detallado de mínimos y puntos silla para poder entender mejor los procesos cinéticos y dinámicos en estos sistemas. Por otro lado, para adsorbatos en superficies, los potenciales de interacción

aste capítulo esta basado en el artículo Potential energy landscape of monolayer-surface systems governed by repulsive lateral interactions: The case of (\$×9)-I-Pt(111), A. Tkatchenko, N. Batina y M. Galván, Phys. Rev. Lett. 97, 036102 (2006). 
se conocen para muy pocos casos, en su mayoría para gases nobles en superficies metálicas [4]. Para la mayor parte de sistemas de quimisorción ni siquiera existe un entendimiento fenomenológico de distintos tipos de interacción. La TFD permite obtener una descripción detallada de superficies de energía potencial (SEP) y hoy en día, se usan de forma rutinaria para la investigación de fenómenos de adsorción $[5,6]$. Un problema de la TFD es el costo computacional de los cálculos, que restringe el muestreo a un máximo de $10^{2}-10^{3}$ puntos hasta para sistemas de pocos átomos. Distintas metodologías se han propuesto para la construcción de SEP analíticas basándose solamente en un número limitado de energías TFD [100-102], pero tales esquemas están restringidos a sistemas con un número reducido de dimensiones y requiriendo además un poder computacional considerable.

Por el lado experimental, la gran cantidad de datos disponibles para la estructura de distintos sistemas sustrato-adsorbato [1] enfatiza la relevancia de un estudio teórico de sus superficies de energía potencial. En este capítulo se hace un estudio de la SEP de cuatro átomos de yodo en la celda $(3 \times 3)$ de la superficie $\mathrm{Pt}(111)$ utilizando una combinación de cálculos de TFD y Monte Carlo con un modelo de interacciones ajustado a las energías de primeros principios.

El sistema I-Pt(111) es uno de los más estudiados por las comunidades científicas de superficies y electroquímica $[37,38,59,60,78-82,98,103]$; desde el punto de vista experimental, es fácil de preparar y mantener estable en vacío, aire y solución, lo cual se ha demostrado en una gran variedad de estudios [37]. Experimentalmente, se han determinado cuatro distintas estructuras para I-Pt(111): $(\sqrt{3} \times \sqrt{3}) R 30^{\circ},(\sqrt{7} \times \sqrt{7}) R 19.11^{\circ},(3 \times 3)$-sym y $(3 \times 3)$-asym. La celda $(3 \times 3)$ con cuatro átomos es particularmente interesante, ya que existen dos posibilidades traslacionales con simetría distinta. Los primeros estudios de difracción de electrones de energía baja (LEED por sus siglas en inglés) sobre I-Pt(111) arrojaron datos sobre la existencia de la estructura $(3 \times 3)$. Las simetrías sym y asym no se pudieron distinguir, ya que producen patrones LEED esencialmente idénticos. Los experimentos de microscopía 
de efecto tunel (STM por sus siglas en inglés) en aire realizados por Schardt y colaboradores [59] demostraron la existencia de las estructuras con simetrías sym y asym y mostraron las diferencias en topografía entre las dos. Otros estudios STM posteriores en aire y solución confirmaron la existencia de ambas estructuras y las encontraron en concentraciones similares en la superficie $\mathrm{Pt}(111)[37,80,98]$. Los estudios recientes de difracción de electrones en vacío [81] encontraron evidencia de la estructura $(3 \times 3)$-sym para el grado de cobertura $\theta=4 / 9$; sin embargo, la estructura con la simetría $(3 \times 3)$-asym no se pudo ajustar al espectro LEED experimental.

Debido a estos resultados, nos parece primordial estudiar el sistema $(3 \times 3)-\mathrm{I}-\mathrm{Pt}(111)$ en detalle y racionalizar las diferencias entre los distintos ambientes experimentales.

\subsection{Metodología}

En esta sección se describen las metodologías empleadas para los cálculos TFD y simulaciones Monte Carlo.

\subsubsection{Cálculos TFD}

Los cálculos TFD que se realizan en este capítulo se basan en el modelo de sistema periódico. La superficie se describe por medio de una losa de cinco capas de átomos de platino con los átomos de yodo (monocapa) adsorbidos por ambos lados de la superficie.

La estructura electrónica se calcula por medio del formalismo de Kohn-Sham [90] utilizando el funcional GGA-PBE para intercambio y correlación [91]. Las ecuaciones de Kohn-Sham se resuelven utilizando una expansión de ondas planas truncada a 40 Ry mediante el programa DFT $++[92]$. Los electrones internos de los átomos de platino y yodo no se consideran explícitamente y se describen mediante el uso de pseudopotenciales de Troullier-Martins con conservación de la norma [74]. El uso de cuatro puntos $k$ en la zona irreducible de Brillouin 
se determinó como apropiado para converger la energía a un nivel de un millihartree. La celda unitaria optimizada fue de $8.48 \times 8.48 \times 27 \AA^{3}$. El nivel de teoría descrito aquí da resultados confiables en comparación con los datos experimentales [47].

\subsubsection{Simulaciones Monte Carlo (MC)}

Las simulaciones Monte Carlo se han usado para la descripción de diferentes fenómenos en una gran variedad de investigaciones, tanto en la física, como en la química, así como en la computación y las matemáticas [104]. Existen muchas variantes y combinaciones de este método para distintos propósitos: Monte Carlo clásico, Monte Carlo cuántico y recocido simulado entre otros. En las ciencias físicas y químicas, estos métodos se han usado para tratar fenómenos muy diferentes, como la adsorción en superficies, o como el plegamiento de proteínas y transiciones de fase. El método Monte Carlo también es utilizado frecuentemente en la mecánica estadística para obtener promedios sobre ensambles [105]. Otra de las grandes aplicaciones del método es para la optimización de funciones complejas. Cuando la función a optimizar no tiene una forma analítica y no se pueden aplicar métodos convencionales de optimización, se tiene que utilizar un método heurístico de búsqueda global, como es el Monte Carlo. Existe una variedad de métodos heurísticos, sobre todo para problemas complejos desde el punto de vista computacional, pero Monte Carlo ha sido uno de los más exitosos hasta la fecha.

Para la búsqueda de mínimos globales se utiliza el método de recocido simulado, que se encuentra dentro del conjunto de métodos Monte Carlo. El nombre proviene de la semejanza de éste al proceso de recocido utilizado en la industria metalúrgica. A grandes rasgos, se utiliza el factor de Boltzmann para aceptar algunos pasos que elevan el valor de la función objetivo para poder encontrar el mínimo global y no quedarse en el mínimo local más cercano a las condiciones iniciales. Existe un teorema que demuestra que el recocido simulado encuentra el mínimo global cuando el número de pasos Monte Carlo tiende a infinito, lo 
que le da un sustento formal a este método. Una ventaja de los métodos Monte Carlo es que solamente se necesita conocer la forma de calcular la función objetivo con las variables independientes y no se requieren los gradientes de ésta.

En este capítulo utilizaremos la técnica de Monte Carlo como un optimizador para encontrar mínimos locales de nuestro sistema. Esto es necesario, ya que la expresión de interacción átomo-superficie utilizada tiene una discontinuidad en los gradientes.

\subsection{Resultados y Discusión}

Para determinar los puntos estacionarios del sistema $(3 \times 3)-\mathrm{I}-\mathrm{Pt}(111)$, se hicieron relajaciones de un conjunto uniforme de estructuras en la celda $(3 \times 3)$ con restricción de simetría hexagonal (los parámetros de celda siempre se mantuvieron fijos). Este conjunto de estructuras fue estudiado recientemente desde el punto de vista geométrico [16]. La opción de utilizar puntos de inicio con simetría hexagonal fue motivada por resultados experimentales, donde se encontraron exclusivamente estructuras con esa simetría. Se encontraron tres extremos distintos. Estos se esquematizan en la Figura 4.1, y se denominan $(3 \times 3)$-sym, $(3 \times 3)$-asym y $(3 \times 3)$-zigzag. ${ }^{a}$ Las primeras dos estructuras se conocen muy bien a partir de distintos estudios experimentales $[59,80,81,98]$, mientras que la tercera no se ha observado para I$\mathrm{Pt}(111)$. Aunque es dificil de ubicar en la figura, las estructuras sym y asym se caracterizan por tener un orden hexagonal esencialmente perfecto dentro de la celda $(3 \times 3)$, mientras que los átomos de yodo situados cerca de los sitios $f$ y $h$ en la estructura $(3 \times 3)$-zigzag se relajan alrededor de $0.3 \AA$ del arreglo hexagonal perfecto.

Con la finalidad de clasificar los puntos extremos, se analizaron los eigenvalores de la matriz Hessiana. Esta se obtuvo por medio de la evaluación númerica de las segundas derivadas

\footnotetext{
${ }^{2}$ En la Figura 4.1c se puede observar que si conectamos los átomos en los sitios $f$ y $h$, se forma un patrón de zig-zag. Se puede ver que la estructura zigzag se caracteriza por la relajación de geometría más notable dentro de la celda unitaria cuando se compara con las estructuras sym y asym.
} 

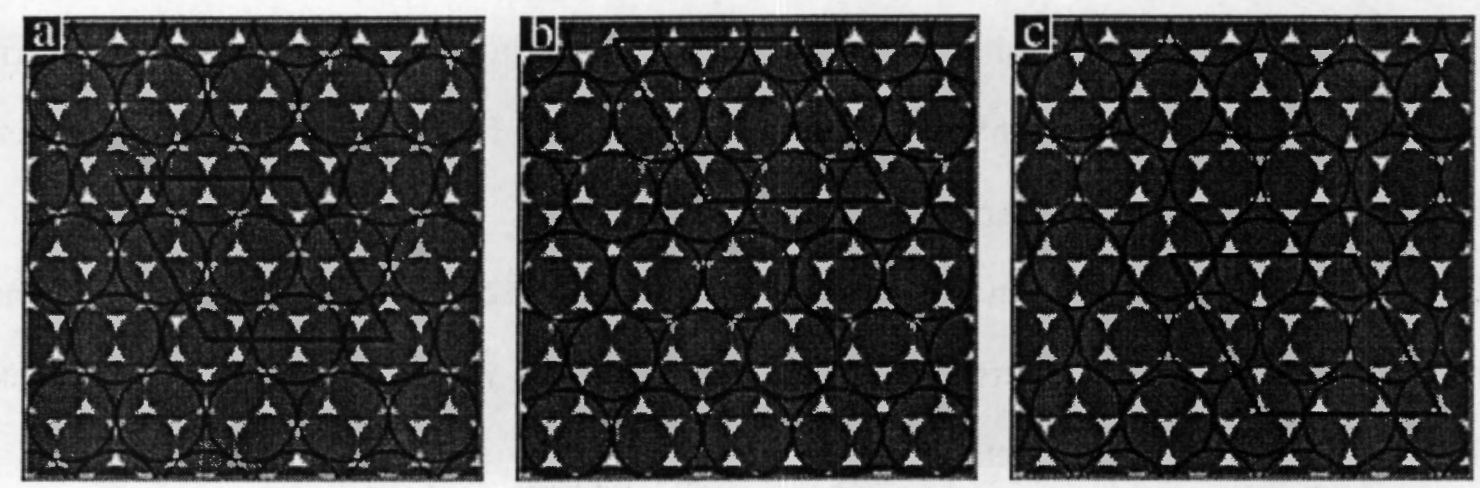

Figura 4.1: Modelos de las tres estructuras en la celda $(3 \times 3)$. La figura muestra dos capas; los círculos negros indican los átomos de yodo y los círculos grises llenos representan la primera capa de la superficie $\mathrm{Pt}(111)$. La celda $(3 \times 3)$ se indica por un rombo en los tres casos. (a) Estructura $(3 \times 3)$-sym con un átomo en el sitio $a$ y tres átomos en los sitios b. (b) Estructura $(3 \times 3)$-asym con un átomo en el sitio $f \mathrm{y}$ tres átomos en sitios asimétricos. (c) Estructura $(3 \times 3)$-zigzag con un átomo en el sitio $f$, un átomo en el sitio $h$ y dos átomos en sitios asimétricos (diferentes que en la estructura $(3 \times 3)$-asym).

para los cuatro átomos de yodo en la celda unitaria. El análisis de los eigenvalores mostró que las estructuras $(3 \times 3)$-sym y $(3 \times 3)$-zizgag son mínimos, mientras que $(3 \times 3)$-asym es un punto silla. Las energías de adsorción de estas estructuras y sus parámetros geométricos se presentan en la Tabla 4.1. La geometría muestra la tendencia esperada intuitivamente [47]: los yodos situados entre tres átomos de la superficie se caracterizan por tener la posición de equilibrio vertical más baja y los sitios $b$ y $a$ se caracterizan por tener distancias verticales mas grandes. Se puede definir una coordenada de reacción que conecta las estructuras sym y asym. Esta se caracteriza por un movimiento colectivo de los cuatro átomos en la celda unitaria siguiendo a la trayectoria lineal en el plano $X-Y$ que conecta el yodo en el sitio $b$ con el sitio $f$ más cercano. Si se permite la relajación de las posiciones verticales, se encuentra por medio de cálculos TFD que la energía de adsorción decrece de forma monótona a lo largo de esta trayectoria que conecta las estructuras sym y asym, lo que confirma el hecho de que $(3 \times 3)$-asym es en realidad un punto silla. Debido a la relajación atómica notable en la celda unitaria para la estructura zigzag, no se puede establecer una trayectoria equivalente 
Tabla 4.1: Las energías de adsorción GGA y los parámetros estructurales $X, Y, Z$ de los cuatro átomos de yodo para las tres estructuras del sistema $(3 \times 3)-\mathrm{I}-\mathrm{Pt}(111)$. Las energías se reportan en $\mathrm{kcal} / \mathrm{mol}$, los parámetros $X-Y$ en unidades de celda unitaria y las posiciones $Z$ en $\AA$ y se miden desde la capa más cercana de la superficie Pt(111).

\begin{tabular}{lccccc} 
& Ad & $I_{1}(X, Y, Z)$ & $I_{2}(X, Y, Z)$ & $I_{3}(X, Y, Z)$ & $I_{4}(X, Y, Z)$ \\
\hline$(3 \times 3)$-sym & 217.9 & $(-0.001,-0.001,2.774)$ & $(0.503,-0.007,2.255)$ & $(-0.005,0.503,2.276)$ & $(0.504,0.505,2.248)$ \\
$(3 \times 3)$-asym & 210.0 & $(-0.112,0.222,2.091)$ & $(0.389,0.220,2.499)$ & $(-0.117,0.725,2.483)$ & $(0.391,0.726,2.490)$ \\
$(3 \times 3)$-zigzag & 212.2 & $(0.078,0.002,2.612)$ & $(0.587,-0.002,2.612)$ & $(0.099,0.472,2.204)$ & $(0.564,0.534,2.129)$ \\
\hline
\end{tabular}

que conecte las estructuras sym o asym con la zigzag.

Considerando que $(3 \times 3)$-sym y $(3 \times 3)$-asym son las únicas estructuras que se han observado en los experimentos $[59,80,81,98]$, a primera vista es sorprendente que $(3 \times 3)$ asym sea un punto silla y $(3 \times 3)$-zizgag un mínimo local. Los estudios experimentales en solución y aire encontraron una mezcla de $(3 \times 3)$-sym y $(3 \times 3)$-asym en la superficie $\mathrm{Pt}(111)$. En contraste, un estudio reciente en vacío [81] indica que no existe evidencia para la existencia de la estructura $(3 \times 3)$-asym. Nuestros resultados de TFD están más cerca a la situación experimental en vacío y esto explica porque el punto silla, $(3 \times 3)$-asym, no se ha observado en esas condiciones. Un estudio experimental reciente en vacío no analizó la posibilidad de formación de la estructura $(3 \times 3)$-zizgag, ya que no se había encontrado anteriormente [81].

Para determinar la razón de la estabilización de las estructuras observadas en aire, solución y vacío, utilizamos una expresión simple para la energía potencial del sistema. Como en muchos otros estudios [4,6], separamos el potencial de interacción para I-Pt(111) en las partes sustrato-adsorbato y adsorbato-adsorbato:

$$
E=\sum_{i}^{N}\left(V\left(x_{i}, y_{i}\right)+\sum_{j>i}\left(A \exp \left(-B r_{i j}\right)\right)\right)
$$

donde $r_{i j}$ es la distancia entre las partículas $i$ y $j$, mientras que $A$ y $B$ son parámetros. $N$ es igual a 4 para la celda $(3 \times 3)$ con grado de cobertura $\theta=4 / 9$. La segunda sumatoria 
se trunca después de $15 \AA$, debido a que incluir un radio de corte de más largo alcance no muestra ningún efecto apreciable sobre los resultados.

Se considera una interacción repulsiva exponencial entre los átomos de yodo en la monocapa adsorbida. Aunque claramente es una simplificación, varios estudios experimentales apuntan a que la interacción efectiva entre los átomos de yodo es repulsiva en un intervalo grande de cobertura [82]. Más aún, la celda $(3 \times 3)$ con grado de cobertura $\theta=4 / 9$ es la más comprimida que se observa en los experimentos ( $\mathrm{a} \theta=4 / 9$ se le dice grado de cobertura de saturación). Para asignar límites a los parámetros $A$ y $B$, hicimos cálculos de yodo adsorbido en el sitio $f$ en la celda $(\sqrt{3} \times \sqrt{3}) R 30^{\circ} \operatorname{con} \theta=\frac{1}{3}$ y en la celda $(1 \times 1) \operatorname{con} \theta=1$. Utilizamos la energía de adsorción de la estructura $(\sqrt{3} \times \sqrt{3}) R 30^{\circ}$ para fijar el parámetro $A$, mientras que dejamos que el parámetro $B$ varíe para simular el efecto de diferentes grados de repulsión entre los átomos de yodo en la monocapa. Se usaron los siguientes valores para $A(\mathrm{kcal} / \mathrm{mol})$ y $B$ (unidades de celda unitaria ${ }^{-1}$ ): $A=8 \times 10^{5}$ y $4.5 \leq B \leq 5$. Un valor menor de $B$ significa una repulsión mayor. Mostraremos enseguida que la manipulación de este único parámetro será suficiente para satisfacer los propósitos de nuestro estudio.

La interacción sustrato-adsorbato $V(x, y)$ para el átomo de yodo con la superficie $\mathrm{Pt}(111)$ se representa por un potencial esférico, $V(x, y)=k Z+b$, donde $Z=$ $\left(\sqrt{\left(R_{1}+R_{2}\right)^{2}-\left(x-x_{s}\right)^{2}-\left(y-y_{s}\right)^{2}}\right)$, el cual da un acuerdo asombroso con las estructuras experimentales del sistema I-Pt(111) $[15,16]$. La cantidad entre paréntesis es la distancia de equilibrio $Z$ del átomo de yodo medida desde la primera capa de la superficie metálica, $R_{1}$ es el radio metálico de los átomos de superficie, $R_{2}$ es el radio de los átomos del adsorbato y $\left(x_{s}, y_{s}\right)$ es la posición del átomo de sustrato más cercano. Los parámetros $k$ y $b$ se ajustan a las energías obtenidas desde los cálculos de primeros principios. Nótese que $R_{2}$ se ajusta para representar las distancias de equilibrio de los átomos del adsorbato. El parámetro $b$ solamente se necesita para obtener valores absolutos de la energía. Por lo tanto, $k$ es el único parámetro que se necesita ajustar para el potencial de interacción átomo-superficie. 


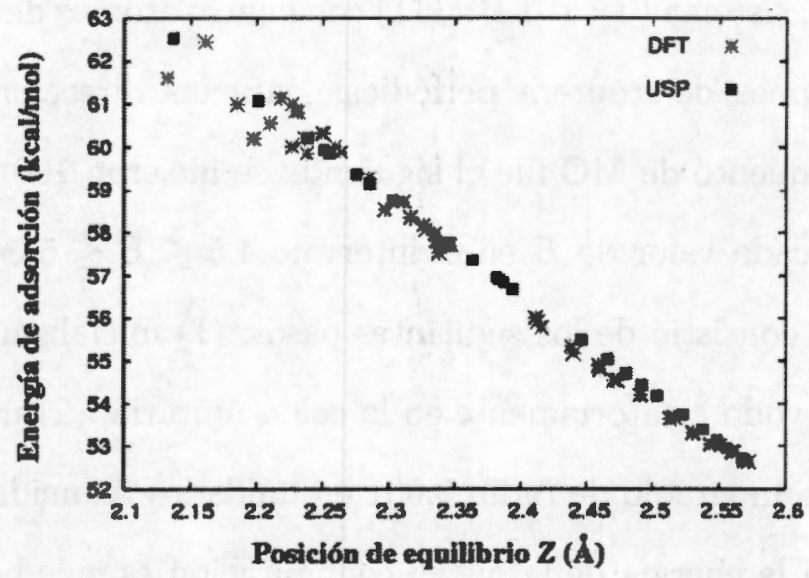

Figura 4.2: La energía de adsorción contra la posición de equilibrio $Z$ para 50 sitios distintos para el átomo de yodo en la celda (3x3)-Pt(111). La posición de equilibrio se mide a partir de la primera capa de la superficie. Los cálculos de TFD se hicieron con un modelo de tres capas para $\mathrm{Pt}(111)$ con el átomo de yodo adsorbido de un solo lado. El potencial esférico (USP) ajustado a los valores de energía y posición vertical de dos sitios ( $f$ y a) reproduce los cálculos de TFD. Nótese la dependencia casi perfecta $E(Z)$, la cual se rompe solamente cerca de los sitios $f-h$, los cuales son degenerados para un modelo extendido de superficie(vea Ref. [47]).

Este parámetro se relaciona directamente con la corrugación del potencial. Se ajustaron los parámetros del potencial esférico con las energías de adsorción y distancias de equilibrio $Z$ para los sitios $f$ y a utilizando cálculos del capítulo anterior. Los valores de los parámetros del modelo esférico utilizado aquí son $R_{1}=1.3875 \AA, R_{2}=1.8122 \AA, k=-22.8140$ $\mathrm{kcal} / \mathrm{mol}, b=96.9091 \mathrm{kcal} / \mathrm{mol}$. En la Figura 4.2, se muestra el excelente desempeño del potencial esférico comparado con los cálculos de TFD (vea el pie de la Figura 4.2 para más detalles). La relación lineal entre $E$ y $Z$ obtenida en los cálculos de TFD es sorprendente y sería interesante investigar este comportamiento para otros sistemas sustrato-adsorbato, donde la superficie posee un arreglo bien definido.

De acuerdo a la Ecuación 4.1, una vez definidos los parámetros para $V(x, y)$ y la constante $A$, la interacción es una función directa de un solo parámetro - $B$, el cual define diferentes regímenes de repulsión e induce deformaciones de la SEP como demostraremos más adelante. Se realizaron simulaciones Monte Carlo con el potencial de interacción de la Ecuación 4.1, 
explorando la SEP del sistema $(3 \times 3)-\mathrm{I}-\mathrm{Pt}(111)$ con cuatro átomos de yodo por celda unitaria. Se utilizaron condiciones de frontera periódicas para las direcciones $X$ y $Y$ de la celda unitaria, y el procedimiento de MC fue el siguiente: se hicieron 100 corridas MC (sin factor de Boltzmann) para cada valor de $B$ en el intervalo $4.5 \leq B \leq 5$ con incrementos de 0.01 . Cada simulación MC consistió de los siguientes pasos: (1) inicializar las posiciones $X-Y$ de los cuatro átomos de yodo aleatoriamente en la celda unitaria, (2) mover uno de los átomos de forma aleatoria en un círculo de radio 0.001 en unidades reducidas de celda unitaria, (3) el paso es aceptado si la energía de la nueva configuración es más baja que la energía de la configuración inmediata anterior, (4) repetir pasos (2)-(3) hasta lograr la convergencia. La configuración final se acepta si la energía no cambia en más de $10^{-4}$ en 5000 pasos Monte Carlo. El número total de pasos en la optimización Monte Carlo varia de 100000 a 200000.

Para $B<4,74,(3 \times 3)$-sym y $(3 \times 3)$-asym fueron los únicos mínimos, mientras que para $B \geq 4.74,(3 \times 3)$-sym y $(3 \times 3)$-zizgag fueron obtenidas exclusivamente. No se pudo encontrar ningún valor de $B$ alrededor de 4.74 que mostrara la existencia de las tres estructuras simultáneamente. Se hicieron corridas adicionales alrededor de $B=4.74$ para verificar que las tres estructuras nunca coexisten para un valor de $B$ dado. Por lo tanto, se puede concluir que la deformación en la SEP alrededor de $B=4.74$ es abrupta, con la estructura asym transformándose en un punto silla y zigzag convirtiéndose en un mínimo local con el aumento del valor de $B$. Es importante recordar que los valores más grandes de $B$ implican menor repulsión. Con esta idea en mente se puede concluir que $(3 \times 3)$-sym es el mínimo global para el intervalo de valores de $B$ utilizados en este estudio, mientras que $(3 \times 3)$-asym es mínimo para repulsión alta y $(3 \times 3)$-zigzag es mínimo para repulsión baja.

Haciendo una comparación de los resultados de simulaciones MC con los datos experimentales mencionados arriba y cálculos de TFD, se observa una tendencia simple. Al parecer el régimen de alta repulsión corresponde a resultados experimentales en aire y solución, mientras que baja repulsión corresponde a resultados de TFD obtenidos en este estudio, los cuales 
se pueden asociar a la situación experimental en vacío y a bajas temperaturas. Siguiendo la misma linea, es importante notar que la estructura $(3 \times \sqrt{3} / 2)$-rect encontrada por Orts y colaboradores [99] en su estudio experimental del sistema $\mathrm{Br}-\mathrm{Pt}(111)$ es esencialmente la misma que (3×3)-zigzag. El hecho de que la estructura zigzag se ha encontrado para $\mathrm{Br}-\mathrm{Pt}(111)$ se puede racionalizar por el radio de van der Waals más pequeño del bromo comparado con yodo, lo cual puede indicar una repulsón efectiva más baja entre los átomos en la monocapa adsorbida.

Es notable que un modelo sencillo, como el presentado en este capítulo, pueda explicar una cantidad importante de resultados experimentales para el sistema $(3 \times 3)-\mathrm{I}-\mathrm{Pt}(111)$. El origen de la repulsión más alta sugiere mayor carga sobre los átomo de yodo en solución, como se ha propuesto para otros sistemas halógeno-metal, y como se puede ver en la Figura 2 de la Ref. [37] y la discusión relacionada. Este efecto es similar a una interacción dipolar repulsiva la cual ya se ha utilizado anteriormente en simulaciones Monte Carlo [106]. Este efecto no contribuye significativamente en vacío, ya que la transferencia de carga es bastante pequeña [47]. En presencia de aire, la repulsión se puede asociar con un efecto de tres cuerpos el cual modifica la interacción yodo-yodo en la monocapa. Para probar el posible efecto del aire, se hicieron cálculos TFD de la monocapa de yodo aislada [sin la superficie Pt(111)] con una molécula de $N_{2}$ interactuando en las posiciones $a$ y $b$. Cuando la molécula se pone 2.5 - $3.5 \AA$ arriba de la superficie, se puede observar un efecto repulsivo sobre la curva de interacción de la monocapa. Este efecto se incrementa a medida que la molécula de $N_{2}$ se acerca más a la monocapa. Los cálculos con la molécula de $N_{2}$ arriba de la monocapa se resumen en la Figura 4.3.

En conclusión, se realizó un estudio combinado de TFD y MC de la superficie de energía potencial de yodo adsorbido en la superficie $\mathrm{Pt}(111)$ en la celda $(3 \times 3)$. Este enfoque resultó ser lo suficientemente poderoso para racionalizar las diferencias en el sistema I-Pt(111) en distintos ambientes experimentales, como aire, solución y vacío. Encontramos una nueva 


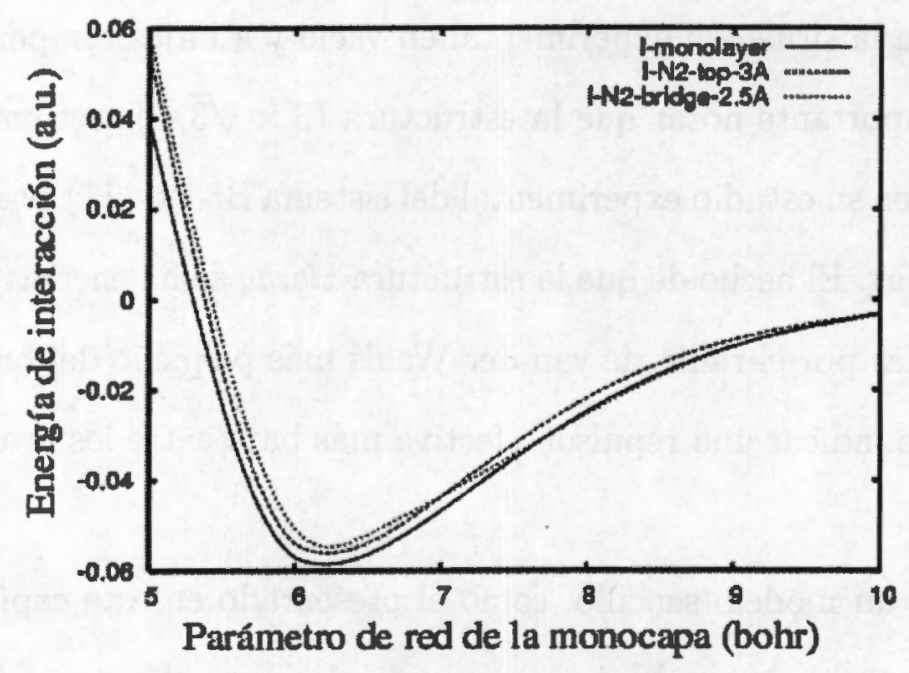

Figura 4.3: La energía de interacción contra el parámetro de red de la monocapa. La energía de interacción se define como la diferencia entre la energía total del sistema para la distancia $d$ y la energía total en el caso aislado ( $d=14 \mathrm{bohr}$ ). Las curvas de energía de interacción se presentan para la monocapa aislada, para la monocapa con la molécula de $N_{2}$ posicionada $3 \AA$ Á sobre en el sitio $a$ y para la molécula de $N_{2}$ posicionada $2.5 \AA$ sobre el sitio $b$.

estructura, llamada $(3 \times 3)$-zigzag y creemos que sería importante realizar estudios experimentales adicionales para confirmar su existencia. 


\section{Conclusiones}

Entre las conclusiones más importantes de esta tesis está el hecho de que la descripción continua del potencial de interacción átomo-superficie es de vital importancia para muchos sistemas monocapa-superficie. Esto se demuestra a lo largo de la tesis para sistemas como metales alcalinos en la superficie $\mathrm{Ag}(111)$ y halógenos en la superficie $\mathrm{Pt}(111)$. Sin duda, el universo de sistemas donde el potencial átomo-superficie requiere de los términos de orden superior de la expansión de Fourier, se puede extender a muchos otros sistemas (monocapas en superficies con un orden bien definido) que no fueron abarcados en esta tesis.

Por otro lado, la retroalimentación entre los modelos analíticos (para la geometría, energía, etc.) y los cálculos de TFD de primeros principios representa una herramienta muy poderosa y aplicable en general a una gama amplia de sistemas monocapa-superficie. En particular, la construcción analítica de monocapas conmensurables en el Capítulo 2 junto con la identificación de términos de Fourier que contribuyen en cada caso permite llevar a cabo cálculos de TFD con estas geometrías y, en principio, obtener el potencial átomo-superficie $V\left(x_{i}, y_{i}\right)$ y las interacciones laterales $L\left(r_{i j}\right)$ (vea la Ecuación 1.1) correctos para una simetría dada. También vale la pena mencionar que los resultados obtenidos para monocapas hexagonales en superficies (111) se pueden extender fácilmente a monocapas o superficies con simetría distinta (mientras estos se puedan definir por un número finito de parámetros de orden).

Otro objetivo importante que se cumplió en este trabajo es la descripción de algunos sis- 
temas reales, como el comportamiento epitaxial rotacional para metales alcalinos en $\mathrm{Ag}(111)$ o la energética de la adsorción de yodo sobre Pt(111). En particular, el logro más trascendental es la determinación del parámetro que controla la existencia de las estructuras del sistema (3×3)-I-Pt(111) en distintos ambientes experimentales, como vacío, aire y solución. Creemos que el acuerdo logrado entre la teoría y los experimentos sustentan fuertemente las metodologías utilizadas en esta tesis. 


\section{Perspectivas}

Un aspecto que se tocó muy poco a lo largo de la tesis es la determinación de interacciones laterales en sistemas reales. En el Capítulo 4 se empleó una forma exponencial sencilla para modelar estas interacciones, pero claramente es una aproximación y su uso se puede justificar solamente para cambios pequeños en el grado de cobertura. El esquema de construcción de monocapas conmensurables descrito en el Capítulo 2 permite, en principio, determinar la curva de interacción lateral desde cálculos de primeros principios. Aunque esto requería de un esfuerzo computacional considerable al momento de la realización de esta tesis, hoy en día este estudio se podría realizar.

La importancia de la interacción átomo-superficie es una de nuestras conclusiones más importantes de la tesis, sin embargo los datos de los coeficientes de la expansión de Fourier y los modelos precisos de estas interacciones se conocen para un número reducido de sistemas. Por esto, creemos que sería trascendental realizar cálculos de TFD para obtener los coeficientes de Fourier para una variedad de sistemas y poder estudiar generalidades. Se abre la posibilidad de una clasificación de distintos sistemas en cuanto a su expansión de Fourier, y con esto proponer esquemas cuantitativos de adsorción.

Por otro lado, muchos de los resultados de esta tesis son para monocapas con una simetría impuesta. Sin embargo, una vez que se tiene una expresión (aún aproximada) para las interacciones laterales, se puede relajar la restricción de simetría impuesta de la monocapa y estudiar la superficie de energía potencial en celdas conmensurables. De hecho, este estudio 
ya se hizo para el caso de interacciones laterales de tipo exponencial [107]. También sería importante hacer estudios fenomenológicos sobre la importancia de términos de orden superior en la expansión de Fourier sobre estructuras ordenadas de monocapas sin restricciones de simetría. Una pregunta interesante sería: ¿ Es posible estabilizar monocapas conmensurables (sin restricción de simetría) con un modelo de un solo término de Fourier?

En general, consideramos que se abren muchas nuevas rutas para el estudio de estructuras monocapa-superficie como resultado de las investigaciones planteadas en esta tesis. 


\section{Apéndice A}

\section{Publicaciones del autor}

Publicaciones realizadas por el autor de esta tesis hasta la fecha:

[1] A. Tkatchenko y N. Batina, Unequal-sphere packing model for the structural arrangement of the well-ordered adsorbate-substrate system, Phys. Rev. B 70, 195403 (2004).

[2] A. Tkatchenko y N. Batina, Detailed characterization of (3x3) iodine adlayer on Pt(111) by unequal-sphere packing model, J. Chem. Phys. 122, 094705 (2005).

[3] A. Tkatchenko, N. Batina, A. Cedillo y M. Galván, Charge transfer and adsorption energies in the I-Pt(111) interaction, Surf. Sci. 581, 58 (2005).

[4] A. Tkatchenko y N. Batina, Unequal-sphere packing model for simulation of the uniaxially-compressed iodine adlayer on Au(111), J. Phys. Chem. B 109, 21710 (2005).

[5] A. Tkatchenko y O. A. von Lilienfeld, Adsorption of Ar on graphite using London dispersion forces corrected density functional theory, Phys. Rev. B 73, 153406 (2006).

[6] M. Göthelid, H. von Schenck, J. Weissenrieder, B. Åkermark, A. Tkatchenko y M. Galván, Adsorption site, core level shifts and charge transfer on the $\operatorname{Pd}(111)-I(\sqrt{3} \times \sqrt{3})$ surface, Surf. Sci 600, 3093 (2006).

[7] A. Tkatchenko, N. Batina y M. Galván, Potential energy landscape of monolayersurface systems governed by repulsive lateral interactions: The case of (3×3)-I-Pt(111), Phys. 
Rev. Lett. 97, 036102 (2006).

[8] A. Tkatchenko, Analytic theory of hexagonal monolayer interacting with hexagonal substrate, Phys. Rev. B 74, 035428 (2006).

[9] A. Tkatchenko y N. Batina, Classification of hexagonal adlayer arrangements by means of collective geometrical properties, J. Chem. Phys. 125, 164702 (2006).

[10] A. Tkatchenko, Role of high-order Fourier terms for stability of monolayer-surface structures: Numerical simulations, Phys. Rev. B 74, 235440 (2006).

[11] A. Tkatchenko, Comprehensive study of the potential energy surface minima of a monolayer on (111) surface, Phys. Rev. B 75, 085420 (2007).

[12] A. Tkatchenko, Commensurate monolayers on surfaces: Geometry and ground states, aceptado en Physical Review B. 


\section{Bibliografía}

[1] G. A. Somorjai, Introduction to Surface Chemistry and Catalysis (Wiley, New York, 1994).

[2] A. Zangwill, Physics at Surfaces (Cambridge University Press, Cambridge, 1988).

[3] D. J. Wales, Energy Landscapes: Applications to Clusters, Biomolecules and Glasses (Cambridge University Press, Cambridge, 2003).

[4] L. W. Bruch, M. W. Cole, y E. Zaremba, Physical Adsorption: Forces and Phenomena (Oxford University Press, New York, 1997).

[5] K. Horn y M. Scheffler, Handbook of Surface Science: Electronic Structure (NorthHolland, Elsevier, Amsterdam, 2000).

[6] R. I. Masel, Principles of Adsorption and Reaction on Solid Surfaces (John Wiley \& Sons, Inc., New York, 1996).

[7] H. Arce y W. L. Mochan, J. Phys.: Condens. Matter 5, A101 (1993).

[8] H. Arce, W. L. Mochan, y G. Cocho, Surf. Sci. 294, 108 (1993).

[9] H. Arce, W. L. Mochan, y J. J. Gutierrez, Surf. Sci. 348, 379 (1996).

[10] S. J. Mitchell, G. Brown, y P. A. Rikvold, Surf. Sci. 471, 125 (2001). 
[11] M. T. M. Koper y J. J. Lukkien, Surf. Sci. 498, 105 (2002).

[12] E. A. Wood, J. Appl. Phys. 35, 1306 (1964).

[13] A. D. McLachlan, Mol. Phys. 7, 381 (1964).

[14] W. A. Steele, Surf. Sci. 36, 317 (1973).

[15] A. Tkatchenko y N. Batina, Phys. Rev. B 70, 195403 (2004).

[16] A. Tkatchenko y N. Batina, J. Chem. Phys. 122, 094705 (2005).

[17] A. Tkatchenko y N. Batina, J. Phys. Chem. B 109, 21710 (2005).

[18] A. Tkatchenko y N. Batina, J. Chem. Phys. 75, 164702 (2006).

[19] G. S. Leatherman y R. D. Diehl, Phys. Rev. B 53, 4939 (1996).

[20] K. Kern, Phys. Rev. Lett. 35, 8265 (1987).

[21] B. M. Ocko, G. M. Watson, y J. Wang, J. Phys. Chem. 98, 897 (1994).

[22] M. A. van Hove y S. Y. Tong, Surface Crystallography by LEED (Springer, Berlin, 1979).

[23] M. A. van Hove, W. H. Weinberg, y C.-M. Chan, Low Energy Electron Diffraction (Springer-Verlag, Berlin, 1986).

[24] O. Biham, L.-W. Chen, y G. Vidali, Surf. Sci. 287, 815 (1993).

[25] A. Patrykiejew, S. Sokolowski, y K. Binder, Surf. Sci. Rep. 37, 207 (2000).

[26] A. Patrykiejew, S. Sokolowski, y K. Binder, J. Chem. Phys. 115, 983 (2001).

[27] A. C. Hillier y M. D. Ward, Phys. Rev. B 54, 14037 (1996). 
[28] D. E. Hooks, T. Fritz, y M. D. Ward, Adv. Mater. 13, 227 (2001).

[29] A. D. Novaco y J. P. McTague, Phys. Rev. Lett. 38, 1286 (1977).

[30] A. D. Novaco y J. P. McTague, Phys. Rev. B 19, 5299 (1979).

[31] D. L. Doering, J. Vac. Sci. Technol. A 3, 809 (1985).

[32] F. Grey y J. Bohr, Europhys. Lett. 18, 717 (1992).

[33] F. Grey y J. Bohr, in Phase Transitions in Surface Films 2, edited by H. Taub, G. Torzo, H. J. Lauter, y S. C. Fain, p. 83, Plenum Press, New York, 1991.

[34] H. Shiba, J. Phys. Soc. Jpn. 46, 1852 (1979).

[35] H. Shiba, J. Phys. Soc. Jpn. 48, 211 (1980).

[36] G. S. Leatherman y R. D. Diehl, Phys. Rev. B 56, 6970 (1997).

[37] O. M. Magnussen, Chem. Rev. 102, 679 (2002).

[38] C. A. Lucas, N. M. Marković, y P. N. Ross, Phys. Rev. B 55, 7964 (1997).

[39] O. M. Magnussen, B. M. Ocko, R. R. Adzic, y J. X. Wang, Phys. Rev. B 51, 5510 (1995).

[40] J. M. Gottlieb y L. W. Bruch, Phys. Rev. B 44, 5750 (1991).

[41] J. M. Gottlieb, Phys. Rev. B 42, 5377 (1990).

[42] G. Vidali y M. W. Cole, Phys. Rev. B 29, 6736 (1984).

[43] A. Tkatchenko, en preparación.

[44] P. Moree y H. J. J. Riele, Math. Comp. 73, 451 (2004). 
[45] C. S. Ogilvy y J. T. Anderson, Diophantine Equations (Wiley, New York, 1988).

[46] H. L. Keng, Introduction to Number Theory (Springer-Verlag, Berlin, 1982).

[47] A. Tkatchenko, N. Batina, A. Cedillo, y M. Galvan, Surf. Sci. 581, 58 (2005).

[48] A. Tkatchenko y M. Galvan, en preparación.

[49] J. L. F. Da Silva, C. Stampfl, y M. Scheffler, Phys. Rev. Lett. 90, 066104 (2003).

[50] J. L. F. Da Silva, C. Stampfl, y M. Scheffler, Phys. Rev. B 72, 075424 (2005).

[51] L. W. Bruch, A. P. Graham, y J. P. Toennies, J. Chem. Phys. 112, 3314 (1999).

[52] A. K. Dham, W. J. Meath, A. R. Allnatt, R. A. Aziz, y M. J. Slaman, Chem. Phys. 142, 173 (1990).

[53] J. F. Zhu, H. Ellmer, H. Malissa, T. Brandstetter, D. Semrad, y P. Zeppenfeld, Phys. Rev. B 68, 045406 (2003).

[54] B. Vogt, B. Kessler, N. Müller, G. Schönhense, B. Schmiedeskamp, y U. Heinzmann, Phys. Rev. Lett. 67, 1318 (1991).

[55] G. Hilgers, M. Pothoff, N. Müller, y U. Heinzmann, Surf. Sci. 322, 207 (1995).

[56] R. D. Diehl, T. Seyller, M. Caragiu, G. S. Leatherman, N. Ferralis, K. Pussi, P. Kaukasoina, y M. Lindroos, J. Phys. Condens. Matter 16, 2839 (2004).

[57] A. Tkatchenko, N. Batina, y M. Galvan, Phys. Rev. Lett. 97, 036102 (2006).

[58] N. Takeuchi, C. T. Chan, y K. M. Ho, Phys. Rev. Lett. 63, 1273 (1989).

[59] B. C. Schardt, S. L. Yau, y F. Rinaldi, Science 243, 1050 (1989).

[60] S. L. Yau, C. M. Vitus, y B. C. Schardt, J. Am. Chem. Soc. 112, 3677 (1990). 
[61] R. D. Diehl y R. McGrath, J. Phys. Condens. Matter 9, 951 (1997).

[62] J. Neugebauer y M. Scheffler, Phys. Rev. B 46, 16067 (1992).

[63] S. A. Wasileski y M. J. Weaver, J. Phys. Chem. B 106, 4782 (2002).

[64] J. Lipkowski, Z. Shi, A. Chen, B. Pettinger, y C. Bilger, Electrochim. Acta 43, 2875 (1998).

[65] M. T. M. Koper y R. A. van Santen, Surf. Sci. 422, 118 (1999).

[66] S. Wang y P. A. Rikvold, Phys. Rev. B 65, 155406 (2002).

[67] S. J. Mitchell y M. T. M. Koper, Surf. Sci. 563, 169 (2004).

[68] C. Stampfl y M. Scheffler, Surf. Rev. Lett. 2, 317 (1995).

[69] A. Kiejna, T. Ossowski, y E. Wachowicz, Surf. Sci. 548, 22 (2004).

[70] M. C. Payne, M. P. Teter, D. C. Allan, T. A. Arias, y J. D. Joannopoulos, Rev. Mod. Phys. 64, 1045 (1992).

[71] N. W. Ashcroft y N. D. Mermin, Solid State Physics (Saunders College Publishing, 1976).

[72] H. J. Monkhorst y J. D. Pack, Phys. Rev. B 13, 5188 (1976).

[73] D. R. Hamman, M. Schluter, y C. Chiang, Phys. Rev. Lett. 43, 1494 (1979).

[74] N. Trouillier y J. Martins, Phys. Rev. B 43, 1993 (1991).

[75] A. Rappe, J. D. Joannopoulos, y P. A. Bash, J. Am. Chem. Soc. 114, 6466 (1992).

[76] A. Rappe, K. Rabe, E. Kaxiras, y J. D. Joannopoulos, Phys. Rev. B 41, 1227 (1990).

[77] J. L. Stickney, S. D. Rosasco, G. N. Salaita, y A. T. Hubbard, Langmuir 1, 66 (1985). 
[78] A. T. Hubbard, Chem. Rev. 88, 633 (1988).

[79] D. G. Frank, O. M. R. Chyan, T. Golden, y A. T. Hubbard, J. Phys. Chem. 97, 3829 (1993).

[80] S. C. Chang, S. L. Yau, B. C. Schardt, y M. J. Weaver, J. Phys. Chem. 95, 4787 (1991).

[81] M. Saidy, K. A. R. Mitchell, S. A. Furman, M. Labayen, y D. A. Harrington, Surf. Rev. Lett. 6, 871 (1999).

[82] M. Labayen, S. A. Furman, y D. A. Harrington, Surf. Sci. 525, 149 (2003).

[83] A. Ignaczak y J. A. N. F. Gomes, J. Electroanal. Chem. 420, 71 (1997).

[84] M. T. M. Koper y R. A. van Santen, Surf. Sci. 422, 118 (1999).

[85] F. L. Hirshfeld, Theo. Chim. Acta 44, 129 (1977).

[86] R. F. Nalewajski y R. G. Parr, Proc. Natl. Acad. Sci. USA 97, 8879 (2000).

[87] R. F. Nalewajski, Chem. Phys. Phys. Chem. 4, 1710 (2002).

[88] F. de Proft, C. V. Alsenoy, A. Peeters, W. Langanaeker, y P. Geerlings, J. Comput. Chem. 23, 1198 (2002).

[89] F. de Proft, R. Vivas-Reyes, A. Peeters, C. V. Alsenoy, y P. Geerlings, J. Comput. Chem. 24, 463 (2003).

[90] R. G. Parr y W. Yang, Density Functional Theory of Atoms and Molecules (Oxford University Press, Oxford, 1989).

[91] J. Perdew, K. Burke, y M. Ernzerhof, Phys. Rev. Lett. 77, 3865 (1996). 
[92] S. Ismail-Beigi y T. Arias, Comp. Phys. Comm. 128, 1 (2000).

[93] A. Kokalj y M. Causa, J. Phys. Condens. Matter 11, 7463 (1999).

[94] R. W. G. Wyckoff, Crystal Structures (Interscience, New York, 1963).

[95] A. Szabo y N. S. Ostlund, Modern Quantum Chemistry: Introduction to Advanced Electronic Structure Theory (McGraw-Hill, New York, 1982).

[96] R. F. W. Bader, Atoms in Molecules, Int. Series of Manuscripts on Chemistry (Oxford University Press, Oxford, 1994).

[97] J. P. Perdew y A. Zunger, Phys. Rev. B 23, 5048 (1981).

[98] J. Inukai, Y. Osawa, M. Wakisaka, K. Sashikata, Y.-G. Kim, y K. Itaya, J. Phys. Chem. B 102, 3498 (1998).

[99] J. M. Orts, R. Gomez, J. M. Feliu, A. Aldaz, y J. Clavilier, J. Phys. Chem. 100, 2334 (1996).

[100] A. Groß y M. Scheffler, Phys. Rev. B 57, 2493 (1998).

[101] G. J. Kroes, Prog. Surf. Sci. 60, 1 (1999).

[102] L. Soenke, PhD thesis, Fritz-Haber Institute, Berlin, 2001.

[103] J. H. Chen, S. L. Yau, y S. C. Chang, J. Phys. Chem. B 106, 9079 (2002).

[104] D. P. Landau y K. Binder, A Guide to Monte Carlo Simulations in Statistical Physics (Cambridge Univeristy Press, Cambridge, 2000).

[105] D. A. McQuarry, Statistical Mechanics (Harper \& Row, New York, 1976).

[106] I. Abou-Hamad, S. J. Mitchell, T. Wandlowski, P. A. Rikvold, y G. Brown, Electrochim. Acta 50, 5518 (2005). 
[107] A. Tkatchenko, Phys. Rev. B 74, 235440 (2006). 